\title{
Dual Role of a Rubisco Activase in Metabolic Repair
} and Carboxysome Organization

Mirkko Flecken ${ }^{1,3}$, Huping Wang ${ }^{1,3}$, Leonhard Popilka ${ }^{1,2}$, F. Ulrich Hartl ${ }^{1}$, Andreas

Bracher $^{1}$ and Manajit Hayer-Hartt 1 ,*

${ }^{1}$ Department of Cellular Biochemistry, Max Planck Institute of Biochemistry, Am Klopferspitz 18, 82152 Martinsried, Germany

${ }^{2}$ Present address: Capgemini Deutschland GmbH, Olof-Palme-Str. 14, 81829 Munich, Germany

${ }^{3}$ These authors contributed equally

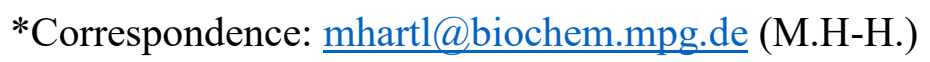




\section{SUMMARY}

Rubisco, the key enzyme of $\mathrm{CO}_{2}$ fixation in photosynthesis, is prone to inactivation by inhibitory sugar phosphates. Inhibited Rubisco undergoes conformational repair by the hexameric AAA+ chaperone Rubisco activase (Rca) in a process that is not well understood. Here we performed a structural and mechanistic analysis of cyanobacterial Rca, a close homolog of plant Rca. In the Rca:Rubisco complex, Rca is positioned over the Rubisco catalytic site under repair and pulls the N-terminal tail of the large Rubisco subunit (RbcL) into the hexamer pore. Simultaneous displacement of the C-terminus of the adjacent RbcL opens the catalytic site for inhibitor release. An alternative interaction of Rca with Rubisco is mediated by C-terminal domains that resemble the small Rubisco subunit. These domains, together with the N-terminal AAA+ hexamer, ensure that Rca is packaged with Rubisco into carboxysomes. Cyanobacterial Rca is a dual-purpose protein with functions in Rubisco repair and carboxysome organization.

Keywords: Cyanobacteria; Nostoc sp. PCC 7120; Nicotiana tabacum; Rubisco; Rubisco activase; metabolic repair; carboxysome; liquid-liquid phase separation; cryo-electron microscopy; X-ray crystallography. 


\section{INTRODUCTION}

The AAA+ (ATPases associated with diverse cellular activities) chaperone Rubisco activase (Rca) serves as a paradigm of conformational enzyme repair (Bhat et al., 2017b; Mueller-Cajar, 2017). The exclusive client of Rca is the photosynthetic key enzyme Rubisco (ribulose-1,5bisphosphate carboxylase/oxygenase), which is directly or indirectly responsible for all biomass production. Since Rca is required for optimal Rubisco function, understanding its mechanism is important in efforts to enhance photosynthesis with the goal to increasing crop yields (Andralojc et al., 2018; Bailey-Serres et al., 2019; Eva et al., 2019; Sharwood, 2017; Singer et al., 2019; Slattery and Ort, 2019).

Rubisco catalyzes the carboxylation of the 5-carbon sugar ribulose-1,5-bisphosphate (RuBP) in the Calvin-Benson-Basham cycle of photosynthesis. This multistep catalytic reaction produces two molecules of 3-phosphoglycerate as fuel for the synthesis of carbohydrates, fatty acids and amino acids. The most prevalent form of Rubisco (form I) in plants, algae and cyanobacteria is a hexadecamer of eight large ( $\mathrm{RbcL} ; \sim 50-55 \mathrm{kDa})$ and eight small (RbcS, 12$18 \mathrm{kDa}$ ) subunits. The RbcL subunits form a cylindrical core, consisting of four anti-parallel dimers, capped by four RbcS subunits at each end of the cylinder. Two active sites per RbcL dimer unit are located at the interface between the N-terminal domain of one RbcL and the Cterminal domain of the other subunit. In order to become functionally competent, the RuBP binding site must be activated by carboxylation of the active-site lysine and binding of a $\mathrm{Mg}^{2+}$ ion, a process referred to as carbamylation. Upon binding of RuBP, the active site is sealed by a mobile loop from the C-terminal domain (the so-called loop 6) and by the otherwise flexible Cterminal sequence stretching over loop 6, forming a multilayered lid (Duff et al., 2000). Together 
with a loop sequence in the N-terminal domain of the adjacent subunit (the so-called '60s loop'), this generates the physical environment required for electrophilic attack of $\mathrm{RuBP}$ by $\mathrm{CO}_{2}$.

The requirement of Rca arises from the fact that the complex catalytic reaction of Rubisco is error prone, leading to the generation of tight-binding sugar phosphates that inactivate the enzyme. These "misfire" products include xylulose-1,5-bisphosphate (XuBP) and 2,3pentodiulose-1,5-bisphosphate (PDBP) (Parry et al., 2008). Moreover, some plants under low light produce the inhibitory 2-carboxy-D-arabinitol-1-phosphate (CA1P) (referred to as "nighttime" inhibitor). Binding of RuBP to non-carbamylated Rubisco also leads to inactive enzyme (Bracher et al., 2017; Parry et al., 2008; Portis et al., 2008). In all these conditions the release of the inhibitory sugar is mediated by Rca, which couples ATP hydrolysis to structural changes at the active site of Rubisco. Rca proteins have evolved independently in photosynthetic organisms and are associated with the red and green lineages of Rubisco (Bhat et al., 2017b; Mueller-Cajar, 2017; Tabita et al., 2008) (Figure 1A). While divergent in sequence, they all share the canonical AAA + core consisting of an $\mathrm{N}$-terminal $\alpha / \beta$-nucleotide binding subdomain and a $\mathrm{C}$-terminal $\alpha$ helical subdomain (Figure 1A), and function as six-membered ring complexes. The AAA+ chaperones are typically substrate-promiscuous and act by threading flexible terminal sequences or loop segments of their target proteins into the hexamer pore (Olivares et al., 2016; Puchades et al., 2020). Threading is mediated by pore-loops (Figure 1A) that face the central pore and engage the substrate peptide, exerting a pulling force (Avellaneda et al., 2020; de la Pena et al., 2018; Dong et al., 2019; Fei et al., 2020; Ripstein et al., 2020; Rizo et al., 2019; Twomey et al., 2019). A low resolution cryo-electron microscopy (cryo-EM) structure of the Rca from the proteobacterium Rhodobacter sphaeroides $(R s)$ in complex with red-type Rubisco (form 1C) showed that the $R s$ Rca hexamer docks onto Rubisco over one catalytic site (Bhat et al., 2017a). 
$R s$ Rca is positioned so as to allow threading of the extended C-terminal sequence of $R s \mathrm{RbcL}$ into the central pore, facilitating the opening of the catalytic site and release of the inhibitory sugar (Bhat et al., 2017a; Mueller-Cajar et al., 2011). In contrast, the mechanism of Rca in plants and green algae containing form IB Rubisco (Tabita et al., 2008) remains enigmatic, because form IB RbcL lacks the extended C-terminal sequence of red-type RbcL. Moreover, Rca of plants and green algae have a domain N-terminal to the AAA+ core that is required for Rubisco recognition (Figure 1A), and the so-called specificity helix H9 in the $\alpha$-helical subdomain confers specificity for Rubisco proteins from solanaceous (nightshade) and non-solanaceous species (Li et al., 2005; Portis et al., 2008; Wachter et al., 2013). The mechanistic analysis of plant Rca has proven difficult, since the N-domain appears to be flexible and the protein, though active as a hexamer, populates a range of dynamic oligomeric states in vitro (Blayney et al., 2011; Keown and Pearce, 2014; Stotz et al., 2011).

The closest sequence homolog to plant Rca is the 'Rca-like' protein of several cyanobacteria with form IB Rubisco, which is required for elevated Rubisco activity under high light conditions (Li et al., 1993; Li et al., 1999). 'Rca-like' lacks the flexible N-terminal domain but its AAA+ core is highly homologous to eukaryotic Rca, with $\sim 38 \%$ sequence identity and $\sim 70 \%$ similarity (Data S1). A distinguishing feature is the presence of a Rubisco small subunitlike (SSUL) domain, connected to the C-terminus of the AAA+ core via a flexible linker (Lechno-Yossef et al., 2020) (Figure 1A). In cyanobacteria, Rubisco is packaged together with carbonic anhydrase into carboxysomes, membraneless compartments in which high concentrations of $\mathrm{CO}_{2}$ are generated for carbon fixation (Badger and Price, 2003; Price et al., 1998; Turmo et al., 2017). The scaffolding protein CcmM of $\beta$-carboxysomes utilizes multiple SSUL modules to induce Rubisco condensate formation during carboxysome biogenesis (Long et 
al., 2010; Wang et al., 2019). The SSUL domain of 'Rca-like' is highly homologous to these modules ( $\sim 40 \%$ identity and $\sim 65 \%$ similarity).

To obtain insight into the basic mechanism of plant Rca, here we analyzed the structure and function of the 'Rca-like' protein of the cyanobacterium Nostoc sp. PCC 7120 (NosRca). NosRca functions as a bona-fide Rubisco activase, independent of the SSUL domain. We solved the crystal structure of the stable $\operatorname{Nos} \mathrm{Rca} \Delta \mathrm{C}$ hexamer, and obtained its high resolution cryo-EM structure in complex with inhibited Rubisco. The structure of the complex revealed molecular details of the activation mechanism. A key finding is the well-resolved N-terminal amino acid sequence of RbcL inserted into the central pore of the spiral Rca hexamer. Additionally, we identified three interface regions, providing insight into how the Rca interacts with Rubisco and remodels the active site to liberate the inhibitory sugar phosphate. Mutational analysis confirmed that plant Rca also engages the N-terminal RbcL sequence of its cognate Rubisco, establishing $\mathrm{N}$-terminal binding as the basic mechanism for activation of form IB Rubisco. We further show that the SSUL domains of the NosRca hexamer, by binding in a groove at the interface of RbcL dimer units, provide an alternative interaction resulting in network formation with Rubisco. The SSUL domains, together with the N-terminal AAA+ core which provides additional valency, ensure that Rca is packaged with Rubisco into carboxysomes. Cyanobacterial Rca thus combines two completely independent, and mutually non-exclusive functions in Rubisco remodeling (ATPdependent) and carboxysome organization (ATP-independent).

\section{RESULTS}

\section{NosRca Protein is a Rubisco Activase}


The purified recombinant NosRca protein, with and without nucleotide, behaved as a $\sim 260 \mathrm{kDa}$ complex by size-exclusion chromatography coupled to multiangle light scattering (SEC-MALS), consistent with a hexamer (theoretical mass $\sim 280 \mathrm{kDa}$ ) (Figures S1A and S1B, and Table S1). NosRca had a constitutive ATPase activity of $\sim 6.5 \mathrm{~min}^{-1}$ per protomer (Figure 1B). Since the $\mathrm{AAA}+$ module shares a high degree of sequence identity ( $\sim 59 \%$ identity and $\sim 88 \%$ similarity) with the AAA+ modules of Rca from Nicotiana tabacum (NtRca) and Arabidopsis thaliana (AtRca), respectively (Data S2), we tested whether it functions as a Rubisco activase. Because activases are adapted to their cognate Rubisco (Bhat et al., 2017b; Mueller-Cajar, 2017), we recombinantly expressed and purified the Rubisco from Nostoc sp. PCC 7120 (NosRubisco) (Figure S1A) using coexpression of Nostoc chaperonin (NosGroES/EL; see STAR Methods). The purified Nos Rubisco, activated with $\mathrm{CO}_{2}$ and $\mathrm{Mg}^{2+}(\mathrm{ECM})$, catalyzed the carboxylation of $\mathrm{RuBP}$ at a rate of $\sim 4$ molecules of $\mathrm{CO}_{2}$ per active site $\mathrm{s}^{-1}$ (Figures $1 \mathrm{C}$ and $1 \mathrm{D}$ ). However, as shown previously ( $\mathrm{Li}$ et al., 1999), binding of RuBP to the non-activated enzyme did not result in inhibition (Figure S1C), in contrast to plant Rubisco (Parry et al., 2008; Stotz et al., 2011; Wang and Portis, 1992). We therefore tested the effect of the misfire sugar phosphate xylulose-1,5bisphosphate $(\mathrm{XuBP})$ as a competitive inhibitor (Bracher et al., 2015). A 60-80\% inhibition of the carboxylation rate was observed at concentrations of 50 to $150 \mu \mathrm{M}$ XuBP in the presence of $400 \mu \mathrm{M}$ RuBP (Figure 1C), indicating that NosRubisco has a lower affinity for XuBP than plant Rubisco (Pearce, 2006). Addition of Nos Rca partially relieved the inhibition in an ATPdependent manner (Figure 1C), suggesting that NosRca facilitated the release of XuBP from the Rubisco active sites. The effect was only partial, because the XuBP remained in the reaction as a competitive inhibitor. However, XuBP is unlikely to be a physiological inhibitor of NosRubisco, consistent with the absence in Nostoc sp. PCC 7120 of XuBP phosphatase, which serves to 
hydrolyze the XuBP upon release (Bracher et al., 2015). Complete and highly efficient inhibition of Rubisco was obtained with 2-carboxyarabinitol-1,5-diphosphate (CABP), a mimic of the carboxyketone intermediate of the carboxylation reaction, at a concentration equivalent to Rubisco active sites $(0.25 \mu \mathrm{M})$ (Figure 1D). Strikingly, NosRca efficiently reactivated the inhibited Rubisco in the presence of ATP (Figures 1D). Thus, NosRca is a bona fide Rubisco activase, although the endogenous inhibitory sugar phosphate(s) of NosRubisco remains to be identified. Deletion of the C-terminal 123 residues (linker and the SSUL domain) in NosRca $(\operatorname{Nos} \mathrm{Rca} \Delta \mathrm{C})$, resulted in a faster ATPase rate $\left(\sim 9.5 \mathrm{~min}^{-1}\right)$ and slightly more efficient Rubisco reactivation (Figures $1 \mathrm{~B}$ and $1 \mathrm{D}$ ), indicating that the SSUL domain is not required for activase function. The rate of reactivation was dependent on the concentration of NosRca (Figure S1D). Similar ATPase rates were measured in the presence of activated or inhibited Rubisco (Figure 1B), a property similar to plant Rca (Robinson and Portis, 1989).

\section{Crystal Structure of NosRca $\Delta \mathrm{C}$}

To allow a structural comparison of NosRca with plant Rca, we solved the crystal structure of $\operatorname{Nos} \operatorname{Rca} \Delta \mathrm{C}$ (residues 2-291) at $\sim 2.7 \AA$ by Gd-multi-wavelength anomalous diffraction (GdMAD) (Figures S1A and Table S2). The resolution of the isomorphic native structure was $2.45 \AA$ (Table S2). The asymmetric unit contained two subunits of NosRca $\Delta \mathrm{C}$, with chain A (residues 2275) having no nucleotide bound and chain B (residues 2-278) having ADP bound (Figures 2A,

2B and S2A). Note that the ADP bound during recombinant expression, as no nucleotide was added during purification and crystallization.

Overall the structure shows the typical AAA+ fold topology, consisting of the N-terminal Rossmann-fold $\alpha / \beta$ subdomain and a smaller C-terminal $\alpha$-helical subdomain (Figures $2 \mathrm{~A}$ and 
2B). The two NosRca $\Delta \mathrm{C}$ subunits had nearly identical subdomain conformations, but differed in the inter-domain angle between the $\alpha / \beta$ and the $\alpha$-helical subdomains by $\sim 11^{\circ}$ (Figure S2B). Chain A superimposes well with the subunit structures of $N t$ Rca (r.m.s.d. $1.46 \AA$ for 206 matching C $\alpha$ positions) and AtRca (r.m.s.d. $1.96 \AA$ for 219 matching C $\alpha$ positions) (Figures 2C and S2C). The $\alpha / \beta$-subdomains separately match almost perfectly (r.m.s.d. $0.53 \AA$ and $0.52 \AA$, respectively). The structural conservation between NosRca and plant Rca includes the so-called specificity helix H9 ( $\alpha 9)$ in the $\alpha$-helical subdomain (Figures 2A and 2B, and Data S1 and S2). As in the NtRca and AtRca crystal lattices (Hasse et al., 2015; Stotz et al., 2011), the $\alpha / \beta$ subdomain and helix $\mathrm{H} 9$ of the adjacent subunit form a rigid module (Figure S2D). The orientation of helix H9 to the four-helix bundle of the $\alpha$-helical subdomain is flexible (Figure $\mathrm{S} 2 \mathrm{E})$. In the rhombohedral crystal lattice of NosRca $\Delta \mathrm{C}$ the subunits form a hexamer with alternating ADP-bound and nucleotide-free subunits (Figure 2D). Note that NosRca $\Delta \mathrm{C}$ is a hexamer in solution as analyzed by SEC-MALS, both in the absence and presence of added nucleotide (Table S1). The interface between the $\alpha / \beta$ subdomain and $\alpha$-helical subdomain of adjacent subunits contains the nucleotide binding site (Figures 2D and S2A). In contrast to the pore-loops in the helical crystal structures of NtRca and AtRca (Hasse et al., 2015; Stotz et al., 2011), the pore-loops of NosRca $\triangle \mathrm{C}$ are ordered (PL1, residues 67-72; PL2, residues 105-119; PL3, residues 160-165), with the exception of the B chains where PL2 is disordered (Figures 2A, 2B and 2D). The hexamer pore would be wide enough for a sphere of $\sim 12 \AA$ diameter to pass. In summary, the high degree of structural similarity of NosRca to plant Rca supports the identification of NosRca as a Rubisco activase. Thus, NosRca can serve as a useful model to understand the mechanism of plant Rca. 


\section{NosRca Binds the N-terminus of RbcL in the Central Pore}

To understand the mechanism of NosRca in remodeling the inhibited Rubisco, we sought to capture NosRca in the process of Rubisco reactivation by cryo-EM. We incubated CABP inhibited NosRubisco (ECM.CABP) with excess NosRca $\Delta \mathrm{C}$ in the presence of ATP, followed after $10 \mathrm{~s}$ by addition of the slowly hydrolyzing ATP analog, ATP $\gamma \mathrm{S}$ (Figure 3A and STAR Methods), a nucleotide replacement strategy previously used in analyzing the 26S proteasome engaged with substrate (Dong et al., 2019). Cryo-EM analysis and single particle reconstruction yielded one predominant conformation of the NosRca $\Delta \mathrm{C}$ :Rubisco complex (Figures S3A-S3C). We obtained an EM density map of the complex with an overall resolution of $\sim 2.86 \AA$ (Figures S3C, S3D and Table S3). The overall resolution of the Rubisco-subtracted NosRca $\Delta \mathrm{C}$ map was $\sim 3.29 \AA$ (Figures S3C and S3E). NosRca $\Delta \mathrm{C}$ docks onto one corner of the cube-shaped Rubisco (Figure 3B), in a manner resembling the interaction of the prokaryotic red-type Rca from $R$. sphaeroides (Bhat et al., 2017a). The cis surface of the hexamer (Figure 2D) faces Rubisco with the $N$-terminal $\alpha / \beta$-subdomains and the central pore of the hexamer being approximately positioned over one substrate binding pocket of a RbcL anti-parallel dimer unit (RbcL-A and RbcL-B; Figure 3B). The engaged catalytic site is in an open conformation with no discernable density for the inhibitor CABP, while the bound CABP was well-resolved in all other seven pockets of Rubisco (Figure S4A). Thus, the interaction between NosRca $\Delta \mathrm{C}$ and Rubisco captured in the cryo-EM structure represents the end-point of NosRca remodeling before dissociation from Rubisco.

A key feature of the complex was the presence of well-defined density in the Nos Rca $\Delta \mathrm{C}$ hexamer pore, fitting to a 13-residue peptide in an extended conformation (Figure 3C). This finding suggested a mechanism similar to that of red-type proteobacterial Rca, which engages the 
flexible C-terminus of RbcL (Bhat et al., 2017a; Mueller-Cajar et al., 2011). However, the Cterminal sequence of RbcL-B was resolved up to residue Glu471 in the Rubisco structure, with only six residues (residues 472-477) missing in the EM density (Figures S4B and S4C), thus excluding engagement of the C-terminus by the NosRca central pore. Strikingly, we found that the N-terminal sequence of subunit RbcL-A residues Ser2 to Tyr14 fitted into the peptide density spanning the pore (Figure 3C). The next resolved N-terminal residue on RbcL-A was Thr24, with residues 15-23 connecting to the peptide in the pore being disordered (Figures S4B and S4D). In all other RbcL subunits the first ordered N-terminal residue is Gly13. We noted that the Nterminal sequence of NosRbcL consists of alternating small and bulky side-chains (Figure 3C). Interestingly, this pattern is conserved at the N-terminus of green-type form IB RbcL (Tabita et al., 2008) of plants, green algae and other cyanobacteria (Figure S4E).

To test the importance of the $\mathrm{N}$-terminus of $\mathrm{RbcL}$ for the reactivation reaction, we generated a truncated $\operatorname{Nos}$ Rubisco mutant lacking 12 residues at the $\mathrm{N}$-terminus $\left(\operatorname{Nos}_{8} \mathrm{~L}_{8} \mathrm{~S}_{8} \Delta \mathrm{N}\right)$ (Figure $\mathrm{S} 1 \mathrm{~A}$ ). $\operatorname{Nos}_{8} \mathrm{~L}_{8} \Delta \mathrm{N}$ exhibited wild-type carboxylation activity, but could no longer be reactivated by NosRca upon inhibition with CABP (Figure 3D). To determine whether this mechanism is conserved in plants, we produced the analogous $\mathrm{N}$-terminal RbcL truncation in Rubisco of $N$. tabacum $\left(N t \mathrm{~L}_{8} \mathrm{~S}_{8} \Delta \mathrm{N}\right)$ by recombinant expression in the presence of chloroplast chaperonins and assembly factors (Aigner et al., 2017; Lin et al., 2019; Wilson et al., 2019) (Figure S1A and STAR Methods). $N t \mathrm{~L}_{8} \mathrm{~S}_{8} \Delta \mathrm{N}$ was carboxylation active but the RuBP inhibited enzyme (E.RuBP) could not be reactivated by $N t$ Rca in contrast to wild-type Rubisco (Figures 3E and S1A, and STAR Methods). Thus, consistent with their high structural homology, engagement of the N-terminal RbcL sequence is important for remodeling by both Nos Rca and plant Rca. 


\section{Pore-loop Interactions with the Rubisco N-terminal Peptide}

The Rubisco-bound NosRca $\Delta \mathrm{C}$ hexamer adopted a helical "split-washer" conformation, with subunit 1 (Rca1; yellow) located closest to and Rca6 (blue) farthest from Rubisco (Figure 4A). Nucleotide density was present in all six nucleotide binding pockets of NosRca, ADP at the Rca1-Rca2 interface and ATP/ATP $\gamma \mathrm{S}$ in the other sites (Figure S5A). The ATP/ATP $\gamma \mathrm{S}$ was positioned close to the well-resolved side-chains of the arginine residues, Arg169 and/or Arg172, from the subsequent subunit (Figure S5A). These arginines have been implicated in ATPhydrolysis in other AAA+ proteins, functioning as the so-called 'arginine fingers' (Puchades et al., 2020). The EM density of both arginines is less well defined at the Rca6-Rca1 interface, the split ends of the NosRca spiral (Figure S5A), perhaps reflecting the presence of mixed conformations due to on-going ATP hydrolysis.

The three pore-loops PL1 (residues 67-72), PL2 (residues 105-119) and PL3 (residues 160-165) (Data S2) were resolved in all NosRca subunits in the EM-density map (Figures S5BS5D), adopting a staircase arrangement. Close examination of the bound peptide revealed that the small side-chains of RbcL-A residues Ala4, Thr6, Thr8 and Thr10 point into successive binding pockets in the central pore formed between the pore-loops PL1 and PL2 of adjacent subunits Rca2 to Rca6 (Figures 4B, S5B and S5C). The ADP-bound Rca1 is the only subunit not in contact with the peptide. The bulkier side-chains of Gln5, Lys7, Gln9 and Lys11 point into the pore solvent channel (Figure 4B). Note that Ser2 and Tyr3, protruding from the trans side of the pore, also conform to this pattern of alternating small and bulky side-chains, while the pattern does not continue beyond residue 12 (Figure S4E). 
The bound peptide buries a substantial surface area of $1018 \AA^{2}$ on NosRca. The contacts to the bound peptide are mediated by van der Waals interactions from both PL1 and PL2 (Figure 4C). In addition, the peptide backbone is in hydrogen-bond distance to the carbonyl group of Pro69 of PL1 in Rca3 to Rca6 (Figures 4C and S5E). Interestingly, the highly conserved aromatic residue Tyr116 in PL2, which is essential in plant Rca (Tyr188 in NtRca) (Stotz et al., 2011) (Data S2), does not make direct contact with the peptide (Figure S5F). In the ATP-bound subunits Rca2 to Rca6, Tyr116 instead forms a hydrogen-bond with Gln121 in the subsequent subunit, resulting in a network of interactions that rigidifies the central pore (Figure S5F). In contrast, the position of Tyr116 is ill-defined in the ADP-bound Rca1. PL3, which makes no contact with the bound peptide (Figure S5D), is positioned between the nucleotide binding site and PL2, suggesting that it may be involved in allosteric regulation via the spatially adjacent arginines Arg169 and Arg172.

\section{Details of the NosRca $\Delta$ C:Rubisco Interface and Remodeling Mechanism}

Beyond engaging the N-terminal sequence of RbcL, Nos Rca contacts Rubisco at three regions covering a total of $1445 \AA^{2}$ (Figures 5A-5E). Interface I (419 $\left.\AA^{2}\right)$ is formed between Rca5/Rca6 and RbcL-A, and has high surface shape complementarity (Figures 5A and 5B). Rca5 residues Leu244 and Asn248 of the specificity helix H9 as well as Leu250, and Rca6 residues Va191 and Gly93 make van der Waals contacts with Glu52, Ile88, Pro90, Pro92 and Asp95 of RbcL-A (Figure 5B). Arg92 in helix $\alpha 3$ of Rca6 forms a salt bridge with Glu52 of RbcL-A, and an intramolecular van der Waals interaction with Glu57 (Figure 5B). Interfaces II and III involve the $\alpha 4-\beta 4$ loop of Rca1 and Rca2, respectively (Figures 5C-5E). In interface II (600 $\left.\AA^{2}\right)$, subunit Rcal inserts between the RbcL-A and RbcL-B antiparallel dimer (Figure 5C). The conformation 
of the protruding $\alpha 4-\beta 4$ loop is rigidified by an intramolecular salt bridge formed between $\operatorname{Arg} 83$ and Asp144, with Pro140 having a further stabilizing effect on the loop. The $\alpha 4-\beta 4$ loop contacts Trp67 of the substrate binding pocket, which is located in the 60s loop (residues 60-69) of RbcLA. In addition, Tyr143 of Rcal makes van der Waals contacts with Gln46, Lys129 and Ala130 of RbcL-A (Figure 5C). Note that there is also a weak interaction between Val91 of Rca1 and the Cterminal residues Ile466 and Lys467 of RbcL-B, which might help in keeping the substrate pocket open for unhindered inhibitor release (Figure 5C). In interface III (431 $\left.\AA^{2}\right)$ the $\alpha 4-\beta 4$ loop of Rca2 contacts the RbcS subunits J and P (Figures 5D and 5E). Specifically, Tyr143 of Rca2 engage in van der Waals interactions with the $\beta 1-\alpha 2$ loop (K57-L61) of RbcS-J and Met1 of RbcS-P (Figure 5E). Residues Arg92, Gly93 and Asn248 of interface I, as well as Pro140 and Tyr143 of interface II/III are conserved in plant Rca (Data S1), providing additional support for a conserved mechanism of remodeling of green-type form IB Rubisco.

Next, we performed a mutational analysis of $\operatorname{Nos} \mathrm{Rca} \Delta \mathrm{C}$ to test the functional significance of these interactions. Note that the integrity of the hexamer structure was preserved in the mutant proteins (Table S1). The interface I mutants V91E/R92G/G93N (breaking the Arg92-Glu52 salt bridge and disrupting the van der Waals interactions with Ile88 and Pro90) and L244D/N248A/L250A (disrupting the interaction with Asp95 and Pro92) preserved wild-type ATPase activity, but failed to reactivate the CABP-inhibited Rubisco (Figures 5F and S1A). The interface II/III mutant P140G/Y143A/D144A (disrupting the interaction of the $\alpha 4-\beta 4$ loop with Rubisco and destabilizing the loop) also abolished reactivation, but additionally showed a $\sim 70 \%$ reduced ATPase activity (Figures 5F and S1A). Mutational analyses in various plant Rca proteins have also implicated residues in helix $\alpha 3$, helix $\alpha 9$ and the $\alpha 4-\beta 4$ loop in the interaction with Rubisco (Li et al., 2005; Ott et al., 2000; Portis et al., 2008; Shivhare and Mueller-Cajar, 2017; 
Shivhare et al., 2019), supporting the functional significance of the structurally defined interface regions in the $\operatorname{Nos} \operatorname{Rca} \Delta \mathrm{C}:$ Rubisco complex.

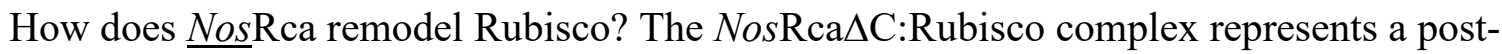
remodeling state, as the Rubisco substrate binding pocket that is engaged by Rca is in an open conformation with no inhibitor bound (Figure S4A). Super-position of the open and closed conformations of the substrate binding pockets revealed the structural changes effected by NosRca (Figure 5G): i) The N-terminal residues 15-23 of RbcL-A are disordered, with no clear discernable EM density, but are well resolved in all other subunits, suggesting that binding the N-terminal sequence (residues 2-14) in the central Rca pore substantially destabilizes this region; ii) As a consequence, the $60 \mathrm{~s}$ loop of RbcL-A, which contributes to stabilizing the substrate binding pocket, is displaced (Figure 5G); iii) In addition, the Rca hexamer - with the bulk of Rcal acting as a wedge - displaces the C-terminal peptide of the adjacent RbcL subunit from its closed position over the substrate pocket (RbcL-B), thereby disrupting its interaction with loop 6. As a result, loop 6 is retracted from its closed position over the substrate binding pocket (Figure 5G). The combined displacement of the 60s loop and loop 6, both of which point to the solvent in the post-remodeling state, results in the complete opening and destabilization of the substrate binding site, facilitating inhibitor release.

\section{Function of the SSUL Domains in Carboxysome Organization}

A distinguishing feature of the NosRca subunit is the presence of the C-terminal SSUL domain (Figure 1A), which is connected to the AAA+ core (residues 2-291) by a $\sim 35$ residue flexible linker. The last structured residue in the EM density map of all NosRca $\Delta \mathrm{C}$ subunits is Asp281. The SSUL domain shares 54-64\% sequence identity with the three SSUL modules of the CcmM 
protein (NosM58) and its shorter variant M35 (NosM35) (Long et al., 2010) (Figure 6A and S6A). The SSUL modules of CcmM function as scaffolding proteins to concentrate Rubisco into a dense condensate prior to encapsulation into the $\beta$-carboxysome (Ryan et al., 2019; Wang et al., 2019). Thus, it seemed plausible that the SSUL domains recruit NosRca into the Rubisco condensate (Lechno-Yossef et al., 2020). Notably, while in M58 and M35 three to five SSUL modules are linked to provide the multi-valency for Rubisco network formation, in NosRca each subunit of the hexamer carries its own SSUL, presumably generating high avidity.

The crystal structure of NosRca-SSUL (NosSSUL, residues 325-414; Figure 1A), solved by MAD at $1.4 \AA$ resolution (Figures 6B, S6B and S6C, and Table S2), confirmed the high degree of similarity to the structure of the SSUL1 module in CcmM of Synechococcus elongatus PCC 7942 (SeSSUL) (r.m.s.d. 1.02-1.04 Å for matching C $\alpha$ positions) (Figure S6D). Like SeSSUL, NosSSUL contains a disulfide bond (Cys363 to Cys380) in the crystal (Figure 6B). The redox state of SeSSUL has been shown to regulate Rubisco condensate formation (Wang et al., 2019), with the cytosol being reducing and the carboxysome interior oxidizing (Chen et al., 2013; Pena et al., 2010; Price et al., 1992).

To investigate whether NosRca has the ability to induce Rubisco condensate formation similar to SeCcmM, we performed an established turbidity assay (Wang et al., 2019). We incubated Rubisco $(0.25 \mu \mathrm{M}$ hexadecamer) with increasing concentrations of reduced NosRca ( 0.125 to $1.0 \mu \mathrm{M}$ hexamer). Turbidity at $340 \mathrm{~nm}$ developed within minutes in a manner dependent on NosRca concentration (Figure 6C), consistent with a recent report for the 'Rcalike' protein from Fremyella diplosiphon (Lechno-Yossef et al., 2020). No turbidity was observed with NosRca $\Delta \mathrm{C}$ (Figure 6C), indicating that the interaction with Rubisco is mediated by the SSUL domains. Neither was turbidity observed with oxidized NosRca $(0.5 \mu \mathrm{M})$, 
indicating that the presence of the disulfide bond in SSUL reduces the affinity for Rubisco (Wang et al., 2019) (Figure 6D and STAR Methods). Note that the oxidized NosRca was as ATPase active and functional in Rubisco remodeling as the reduced protein (Figure S6E). Cryo-EM analysis resolved the interaction of NosRca with Rubisco mediated by NosSSUL at $8.3 \AA$ resolution (Figures S7A-S7D). Docking of the NosSSUL crystal structure and the NosRubisco model into the low resolution EM density map revealed that the SSUL module binds close to the equatorial region of the hexadecamer in a groove between RbcL dimers (Figure S7E), as previously shown for the SeM35-Rubisco complex (Wang et al., 2019).

Interestingly, addition of ATP $\gamma \mathrm{S}$ to reduced NosRca resulted in enhanced turbidity with Rubisco (Figure 6D). However, this effect was still completely dependent on the presence of the SSUL domains (Figure 6D), suggesting the possibility that the AAA+ core modulates the interaction by contributing additional valency. Consistent with such an enhancing effect, addition of ATP $\gamma \mathrm{S}$ also enabled measurable turbidity with oxidized NosRca (Figure 6D).

To determine whether the observation of turbidity reflected demixing of NosRca and Rubisco into phase separated droplets, we N-terminally labeled Rubisco with Alexa Fluor 532 (Rubisco-A532) and NosRca (or NosRca $\Delta$ C) with Alexa Fluor 405 (A405-Rca or A405-Rca $\Delta$ C). Upon incubation of the Rca protein with Rubisco (labeled:unlabeled 1:10), liquid-liquid phase separation (LLPS) into fluorescent droplets was readily observed (Figure 6E). No droplet formation occurred with $\operatorname{Nos} \mathrm{Rca} \Delta \mathrm{C}$ and Rubisco (Figure 6F).

To directly assess the contribution of the AAA+ core to LLPS, we generated a mutant of NosRca with disrupted interface I (mutations V91E/R92G/G93N and L244D/N248A/L250A combined) (NosRca-IF mut). NosRca-IF mut was a stable hexamer in solution (Figure S1A and Table S1). Strikingly, the ability of Nos Rca-IF mut to induce Rubisco condensate formation was strongly 
reduced (Figure 6G), and only very small droplets were observed, despite the presence of the SSUL domains (Figure 6H). Thus, in addition to the SSUL domains, the interaction via the $\mathrm{AAA}+$ core contributes critical valency to the phase separation process. Apparently, both interaction modes cooperate in ensuring packaging of NosRca into carboxysomes together with Rubisco.

\section{DISCUSSION}

\section{Mechanism of Form 1B Rubisco Metabolic Repair}

Our structural and functional analysis of cyanobacterial NosRca, a close homolog of plant Rca, revealed the mechanism of ATP-dependent form 1B Rubisco metabolic repair. In the highresolution cryo-EM structure of the $\operatorname{Nos} \mathrm{Rca} \Delta \mathrm{C}$ :Rubisco complex, NosRca docks onto inhibitorbound Rubisco with its hexamer pore approximately positioned over one of the eight catalytic sites. In contrast to the proteobacterial red-type $R s \mathrm{Rca}$, which engages the extended RbcL Cterminus (Bhat et al., 2017a), NosRca is rotated clockwise by $\sim 50^{\circ}$ degrees, positioning its central pore to bind the N-terminus of RbcL (Figure 7A). Insertion of the N-terminus into the central pore of NosRca is critical for Rubisco activation. This mechanism is likely of general relevance in organisms with green-type form 1B Rubisco (cyanobacteria, green algae and plants), as their RbcL subunits lack the extended C-terminal sequence of red-type RbcL. Indeed, N-terminal truncation of RbcL from tobacco prevented activation of inhibited Rubisco by its cognate Rca.

The complex of NosRca with inhibitor-bound Rubisco, stabilized by a nucleotide substitution strategy, represents an end-state of the remodeling process in which the substrate binding site under repair is open and the inhibitor released. In addition to insertion of the N- 
terminus of one RbcL (RbcL-A) into the Rca hexamer pore, destabilization of the binding pocket involves extensive interactions with Rubisco via three interface regions (Figure 7B).

Underscoring the sequence homology to plant Rca (Data S1), critical residues of these interfaces are structurally conserved and their functional relevance is confirmed by mutagenesis (Figure 7C). Interface I formed by Rca5/Rca6 with RbcL-A includes the specificity helix H9 on Rca5 (Figure 5B). In plants, helix H9 functions in distinguishing Rubisco proteins from solanaceae and non-solanaceae (Portis et al., 2008; Wachter et al., 2013). Notably, the RbcL residues Pro90 and Asp95, which mediate Rca specificity in non-solanaceous plants (e.g. Pro89 and Asp94 in $A$. thaliana and Spinacia oleraces), are conserved in NosRbcL (Li et al., 2005; Ott et al., 2000) (Figure 7C). Pro90 contacts the NosRca backbone at Va191 and Gly93 in the $\alpha 3-\beta 3$ loop of Rca6. Note that in plant Rca sequences this loop is one residue shorter (Data S1 and S2). Asp95 in NosRbcL makes van der Waals contacts to Leu244 in helix H9 of NosRca5 (Figure 7C). Modeling of the AtRca:Rubisco complex based on the NosRca:Rubisco structure suggests that Asp94 in $A t \mathrm{RbcL}$ is in electrostatic interaction with Lys313 in AtRca (the residue equivalent to Leu244 in NosRca) (Figure 7C). This interaction mediates the Rca-Rubisco specificity of nonsolanaceous plants, consistent with previous mutational analyses (Li et al., 2005; Ott et al., 2000; Portis et al., 2008). In tobacco, a solanaceous plant, this electrostatic interaction is reversed, with Lys94 on $N t$ RbcL interacting with Asp316 in NtRca (Figure 7C), and the charge reversal switching the specificity from non-solanaceous to solanaceous (Portis et al., 2008). Another conserved interaction in interface I is the salt bridge Arg92 in NosRca6 with Glu52 in RbcL, which is equivalent to the salt bridges of Lys161 to Glu51 and Lys164 to Glu51 in A. thaliana and tobacco, respectively (Figure 7C). 
Interfaces II and III involve the $\alpha 4-\beta 4$ loop of Rca1 and Rca2. In interface II, the $\alpha 4-\beta 4$ loop of Rcal inserts between the RbcL-A and RbcL-B subunits of the antiparallel dimer containing the catalytic site under repair. In interface III, the $\alpha 4-\beta 4$ loop of Rca2 makes contact with $\mathrm{RbcS}$ subunits $\mathrm{J}$ and $\mathrm{P}$. As a result of these combined interactions, the catalytic site is converted to a completely open state. Specifically, the 60s loop of RbcL-A, and the C-terminal tail and loop 6 of the adjacent RbcL-B subunit, all of which contribute to stabilizing the closed state of the substrate binding pocket, are displaced (Figure 7D). The interaction of Nos Rca with $\mathrm{RbcS}$ is intriguing. RbcS contributes indirectly to stabilizing the sugar substrate in the active site pocket (Bracher et al., 2011) and thus the interaction with NosRca, in addition to providing an anchoring point, may also prevent dissociation of $\mathrm{RbcS}$ while the catalytic site is being destabilized.

\section{Binding of the RbcL N-terminus in the Rca Central Pore}

The N-terminal peptide of RbcL-A (residues 2-14) buries an extensive surface of the NosRca pore, apparently representing an end-state in which Rca spends most of its time during the remodeling cycle. Consistent with this assumption, the NosRca:Rubisco complex was resolved only in one defined state. Presumably, ATP-dependent peptide threading comes to a halt when this stably locked position is reached, thereby limiting further Rca action.

The interactions of the N-terminal residues of RbcL-A with the central Rca pore are remarkably well resolved in the cryo-EM structure. This sequence is characterized by an alternating pattern of small and bulky residues, which is generally conserved in green-type form 1B RbcL. The small side-chains of Ala4, Thr6, Thr8 and Thr10 are pointing into successive binding pockets in the central pore, while Ser2 and Tyr3 protrude from the trans-side of the pore. 
The binding pockets appear to represent an adaptation to the alternating side-chain pattern of the RbcL N-terminus. They are formed by pore-loops PL1 and PL2 of adjacent subunits from Rca2 through Rca6, and are staggered due to the stair-case arrangement of the Rca subunits. In contrast, the extended C-terminal sequence of red-type RbcL does not show a conserved sidechain pattern (Mueller-Cajar et al., 2011). Structural information on the interaction of the redtype RbcL C-terminus with the central pore of its cognate Rca is still missing.

Many $\mathrm{AAA}^{+}$proteins, including $R s \mathrm{Rca}$, contain an aromatic residue (Tyr114 in $R s \mathrm{Rca}$ ) in their canonical PL1 motif (aromatic-hydrophobic-glycine) that is involved in substrate peptide threading (de la Pena et al., 2018; Dong et al., 2019; Fei et al., 2020; Hanson and Whiteheart, 2005; Ripstein et al., 2020; Rizo et al., 2019; Twomey et al., 2019; Wang et al., 2020). The aromatic residues of the six PL1 loops grip and translocate the peptide substrate in a sequencepromiscuous, hydrophobic milieu. Interestingly, NosRca and plant Rca do not have this canonical PL1 motif (Data S2). However, PL2 contains the highly conserved and functionally critical aromatic residue Tyr116 (Tyr188 in NtRca) (Stotz et al., 2011). In the ATP-bound subunits Rca2 to Rca6, Tyr116 hydrogen-bonds with Gln121 in the subsequent subunit, forming a network of interactions that rigidifies the central pore. In contrast, in the ADP-bound Rca1, PL2 is flexible and the position of Tyr116 is not well defined. Thus, it would appear that Tyr116 is unlikely to be involved in the process of peptide insertion in a manner similar to the Tyr in canonical pore-loops PL1. Instead, the formation of pockets between the staggered PL1 and PL2 of adjacent Rca subunits may be the critical feature for sequence specific substrate capture. As suggested for other AAA+ proteins, peptide threading may be mediated by sequential ATP hydrolysis around the hexamer ring (de la Pena et al., 2018; Dong et al., 2019; Fei et al., 2020; Ripstein et al., 2020; Rizo et al., 2019; Twomey et al., 2019; Wang et al., 2020). 


\section{Function of SSUL Domain and AAA+ Core in Carboxysome Organization}

Another important feature of NosRca is the presence of a Rubisco small subunit-like (SSUL) domain flexibly attached to the C-terminus of the AAA+ core in each Rca subunit. These SSUL domains mediate an alternative mode of interaction with Rubisco. They bind into a groove close to the equatorial region of Rubisco between the RbcL dimer units. This interaction has also been shown for the SSUL domains of the carboxysomal scaffolding protein CcmM (Wang et al., 2019), which mediates, via multivalent interactions, the formation of a Rubisco condensate for packaging into carboxysomes. NosSSUL also contains the disulfide bond previously described for the SSUL module in CcmM, and thus its interaction with Rubisco is redox regulated, resulting in reduced affinity under oxidizing conditions.

In contrast to CcmM, which contains 3-5 SSUL modules as a linear fusion, NosRca contains six separate SSUL, one on each subunit, presumably providing high avidity for Rubisco. However, this arrangement may sterically preclude multivalent interactions required for efficient Rubisco network formation. Indeed, we found that critical valency is contributed by the AAA+ core of NosRca, as the mutational disruption of interface I resulted in a nearly complete loss in Rubisco condensate formation, despite the presence of the SSUL domains (Figure 6G). Thus, the seemingly unrelated interactions of NosRca in Rubisco repair and binding to Rubisco via the SSUL domains are linked and must cooperate to ensure Rca packing into carboxysomes (Figure 7E). Upon transfer into the oxidizing environment of the carboxysome, the SSUL-mediated association would then be released in favor of the functional interaction for Rubisco repair via the AAA+ core. It will be interesting to understand the interplay of NosRca and CcmM in Rubisco condensate formation during carboxysome biogenesis. 
The acquisition of the SSUL domain by the NosRca AAA+ core probably occurred in the context of the appearance of carboxysomes about 350 million years ago, long after the primary endosymbiotic event leading to the evolution of chloroplasts (Rae et al., 2013). Efforts to introduce carboxysomes into chloroplasts in order to increase Rubisco efficiency (Hanson et al., 2016; Hennacy and Jonikas, 2020; Long et al., 2016; Rae et al., 2017), will have to consider coexpression of cyanobacterial Rca or attachment of the SSUL domain to plant activase.

\section{AUTHOR CONTRIBUTIONS}

M.F. and H.W. planned and performed most of the experiments. M.F. established and performed all the activase assays and carried out turbidity assays together with H.W. M.F. was responsible for construct development and protein purification, and performed the synthesis of CABP. H.W. performed the cryo-EM structural analysis, including sample screening, grid preparation, data acquisition and single particle reconstructions, and fluorescence microscopy. L.P. contributed to cloning and protein purification, and obtained crystals of NosRca $\Delta \mathrm{C}$ and NosSSUL. A.B. supervised the structural analyses and solved the crystal structures. M.H.-H. conceived the project and participated in data interpretation with F.U.H. and the other authors. M.H.-H and F.U.H wrote the manuscript with input from H.W., A.B. and M.F.

\section{ACKNOWLEDGMENTS}

The technical assistance in the cloning and purification of proteins by S. Gaertner and R. Lange is gratefully acknowledged. We thank F. Bonneau and C. Basquin (Department of Structural Cell Biology) for help with the screening of a suitable buffer for protein purification, G. ThieulinPardo for providing NtRcaR294V and NtRubisco as well as advice on Rca assays, R.H. Wilson 
for advice on expression of cyanobacterial Rubisco, X. Yan for advice on fluorescence microscopy, P. Wendler (University Potsdam) for initial analysis of NosRca by negative-stain EM, D. Cobessi (Institut de Biologie Structurale, Grenoble) for help with X-ray data collection of NosSSUL and D. Bollschweiler for advice with cryo-EM data collection. We are grateful to the staff at FIP (French beamline for Investigation of Proteins) beamline BM30A at the European Synchrotron Radiation Facility (ESRF) and at beamline X06DA at the Swiss Synchrotron Light Source (SLS) in Villigen, Switzerland. Expert support by the Imaging, Crystallization and cryoEM facilities of the Max Planck Institute of Biochemistry is acknowledged.

\section{REFERENCES}

Adams, P.D., Afonine, P.V., Bunkoczi, G., Chen, V.B., Davis, I.W., Echols, N., Headd, J.J., Hung, L.W., Kapral, G.J., Grosse-Kunstleve, R.W., et al. (2010). PHENIX: a comprehensive Pythonbased system for macromolecular structure solution. Acta Crystallogr. D Biol. Crystallogr. 66, 213-221.

Aigner, H., Wilson, R.H., Bracher, A., Calisse, L., Bhat, J.Y., Hartl, F.U., and Hayer-Hartl, M. (2017). Plant Rubisco assembly in E. coli with five chloroplast chaperones including BSD2. Science 358, 1272-1278.

Andralojc, P.J., Carmo-Silva, E., Degen, G.E., and Parry, M.A.J. (2018). Increasing metabolic potential: C-fixation. Essays Biochem. 62, 109-118.

Avellaneda, M.J., Franke, K.B., Sunderlikova, V., Bukau, B., Mogk, A., and Tans, S.J. (2020). Processive extrusion of polypeptide loops by a Hsp100 disaggregase. Nature 578, 317-320.

Badger, M.R., and Price, G.D. (2003). $\mathrm{CO}_{2}$ concentrating mechanisms in cyanobacteria: molecular components, their diversity and evolution. J. Exp. Bot. 54, 609-622.

Bailey-Serres, J., Parker, J.E., Ainsworth, E.A., Oldroyd, G.E.D., and Schroeder, J.I. (2019). Genetic strategies for improving crop yields. Nature 575, 109-118.

Baker, R.T., Catanzariti, A.M., Karunasekara, Y., Soboleva, T.A., Sharwood, R., Whitney, S., and Board, P.G. (2005). Using deubiquitylating enzymes as research tools. Methods Enzymol. 398, 540-554. 
Barta, C., Carmo-Silva, A.E., and Salvucci, M.E. (2011). Rubisco activase activity assays. Methods Mol. Biol. 684, 375-382.

Bhat, J.Y., Miličić, G., Thieulin-Pardo, G., Bracher, A., Maxwell, A., Ciniawsky, S., MuellerCajar, O., Engen, J.R., Hartl, F.U., Wendler, P., et al. (2017a). Mechanism of enzyme repair by the AAA+ chaperone Rubisco activase. Mol. Cell 67, 744-756.e6.

Bhat, J.Y., Thieulin-Pardo, G., Hartl, F.U., and Hayer-Hartl, M. (2017b). Rubisco Activases: AAA+ Chaperones Adapted to Enzyme Repair. Front. Mol. Biosci. 4, 20. doi: 10.3389/fmolb.2017.00020.

Biyani, N., Righetto, R.D., McLeod, R., Caujolle-Bert, D., Castano-Diez, D., Goldie, K.N., and Stahlberg, H. (2017). Focus: The interface between data collection and data processing in cryoEM. J. Struct. Biol. 198, 124-133.

Blayney, M.J., Whitney, S.M., and Beck, J.L. (2011). NanoESI mass spectrometry of Rubisco and Rubisco activase structures and their interactions with nucleotides and sugar phosphates. J. Am. Soc. Mass Spectrom. 22, 1588-1601.

Bracher, A., Sharma, A., Starling-Windhof, A., Hartl, F.U., and Hayer-Hartl, M. (2015). Degradation of potent Rubisco inhibitor by selective sugar phosphatase. Nat. Plants 1, 1-7.

Bracher, A., Starling-Windhof, A., Hartl, F.U., and Hayer-Hartl, M. (2011). Crystal structure of a chaperone-bound assembly intermediate of form I Rubisco. Nat. Struct. Mol. Biol. 18, 875-880.

Bracher, A., Whitney, S.M., Hartl, F.U., and Hayer-Hartl, M. (2017). Biogenesis and metabolic maintenance of Rubisco. Annu. Rev. Plant Biol. 68, 29-60.

Catanzariti, A.M., Soboleva, T.A., Jans, D.A., Board, P.G., and Baker, R.T. (2004). An efficient system for high-level expression and easy purification of authentic recombinant proteins. Protein Sci. 13, 1331-1339.

Chen, A.H., Robinson-Mosher, A., Savage, D.F., Silver, P.A., and Polka, J.K. (2013). The bacterial carbon-fixing organelle is formed by shell envelopment of preassembled cargo. PLoS One 8 , e76127. doi: 10.1371/journal.pone.0076127.

Chen, V.B., Arendall, W.B., 3rd, Headd, J.J., Keedy, D.A., Immormino, R.M., Kapral, G.J., Murray, L.W., Richardson, J.S., and Richardson, D.C. (2010). MolProbity: all-atom structure validation for macromolecular crystallography. Acta Crystallogr. D Biol. Crystallogr. 66, 12-21.

Chifflet, S., Torriglia, A., Chiesa, R., and Tolosa, S. (1988). A method for the determination of inorganic phosphate in the presence of labile organic phosphate and high concentrations of protein: Application to lens ATPases. Anal. Biochem. 168, 1-4.

Collaborative Computational Project, N. (1994). The CCP4 suite: programs for protein crystallography. Acta Crystallogr. D Biol. Crystallogr. 50, 760-763. 
Cowtan, K. (2006). The Buccaneer software for automated model building. 1. Tracing protein chains. Acta Crystallogr. D Biol. Crystallogr. 62, 1002-1011.

de la Fortelle, E., and Bricogne, G. (1997). Maximum-likelihood heavy atom parameter refinement for multiple isomorphous replacement and multiwavelength anomalous diffraction methods. Methods Enzymol. 276, 472-494.

de la Pena, A.H., Goodall, E.A., Gates, S.N., Lander, G.C., and Martin, A. (2018). Substrateengaged 26S proteasome structures reveal mechanisms for ATP-hydrolysis-driven translocation. Science 362, pii: eaav0725. doi: 10.1126/science.aav0725.

Dong, Y., Zhang, S., Wu, Z., Li, X., Wang, W.L., Zhu, Y., Stoilova-McPhie, S., Lu, Y., Finley, D., and Mao, Y. (2019). Cryo-EM structures and dynamics of substrate-engaged human 26S proteasome. Nature 565, 49-55.

Duff, A.P., Andrews, T.J., and Curmi, P.M. (2000). The transition between the open and closed states of rubisco is triggered by the inter-phosphate distance of the bound bisphosphate. J. Mol. Biol. 298, 903-916.

Emsley, P., and Cowtan, K. (2004). Coot: model-building tools for molecular graphics. Acta Crystallogr. D Biol. Crystallogr. 60, 2126-2132.

Esau, B.D., Snyder, G.W., and Portis, A.R., Jr. (1996). Differential effects of N- and C-terminal deletions on the two activities of Rubisco activase. Arch. Biochem. Biophys. 326, 100-105.

Eva, C., Oszvald, M., and Tamas, L. (2019). Current and possible approaches for improving photosynthetic efficiency. Plant Sci. 280, 433-440.

Evans, P. (2006). Scaling and assessment of data quality. Acta Crystallogr. D Biol. Crystallogr. $62,72-82$.

Evans, P.R. (1997). Scala. Joint CCP4 and ESF-EACBM Newsletter 33, 22-24.

Evans, P.R., and Murshudov, G.N. (2013). How good are my data and what is the resolution? Acta Crystallogr. D Biol. Crystallogr. 69, 1204-1214.

Fei, X., Bell, T.A., Jenni, S., Stinson, B.M., Baker, T.A., Harrison, S.C., and Sauer, R.T. (2020). Structures of the ATP-fueled ClpXP proteolytic machine bound to protein substrate. Elife 9, pii: e52774. doi: 10.7554/eLife.52774.

French, G., and Wilson, K. (1978). On the treatment of negative intensity observations. Acta Cryst. $A 34,517-525$.

Gouet, P., Courcelle, E., Stuart, D.I., and Metoz, F. (1999). ESPript: multiple sequence alignments in PostScript. Bioinformatics 15, 305-308. 
Hanson, M.R., Lin, M.T., Carmo-Silva, A.E., and Parry, M.A. (2016). Towards engineering carboxysomes into C3 plants. Plant J. 87, 38-50.

Hanson, P.I., and Whiteheart, S.W. (2005). AAA+ proteins: have engine, will work. Nat. Rev. Mol. Cell Biol. 6, 519-529.

Hasse, D., Larsson, A.M., and Andersson, I. (2015). Structure of Arabidopsis thaliana Rubisco activase. Acta Crystallogr. D Biol. Crystallogr. 71, 800-808.

Hennacy, J.H., and Jonikas, M.C. (2020). Prospects for engineering biophysical $\mathrm{CO}_{2}$ concentrating mechanisms into land plants to enhance yields. Annu. Rev. Plant Biol. doi: 10.1146/annurev-arplant-081519-040100.

Kabsch, W. (2010). XDS. Acta Crystallogr. D Biol. Crystallogr. 66, 125-132.

Kabsch, W., and Sander, C. (1983). Dictionary of protein secondary structure: pattern recognition of hydrogen-bonded and geometrical features. Biopolymers 22, 2577-2637.

Karkehabadi, S., Peddi, S.R., Anwaruzzaman, M., Taylor, T.C., Cederlund, A., Genkov, T., Andersson, I., and Spreitzer, R.J. (2005). Chimeric Small Subunits Influence Catalysis without Causing Global Conformational Changes in the Crystal Structure of Ribulose-1,5-bisphosphate Carboxylase/Oxygenase. Biochemistry 44, 9851-9861.

Keown, J.R., and Pearce, F.G. (2014). Characterization of spinach ribulose-1,5-bisphosphate carboxylase/oxygenase activase isoforms reveals hexameric assemblies with increased thermal stability. Biochem. J. 464, 413-423.

Kerner, M.J., Naylor, D.J., Ishihama, Y., Maier, T., Chang, H.C., Stines, A.P., Georgopoulos, C., Frishman, D., Hayer-Hartl, M., Mann, M., et al. (2005). Proteome-wide analysis of chaperonindependent protein folding in Escherichia coli. Cell 122, 209-220.

Kleywegt, G.T., and Jones, T.A. (1994). A super position. CCP4/ESF-EACBM Newsletter on Protein Crystallogr. 31, 9-14.

Krissinel, E., and Henrick, K. (2007). Inference of macromolecular assemblies from crystalline state. J. Mol. Biol. 372, 774-797.

Larimer, F.W., and Soper, T.S. (1993). Overproduction of Anabaena 7120 ribulose-bisphosphate carboxylase/oxygenase in Escherichia coli. Gene 126, 85-92.

Lechno-Yossef, S., Rohnke, B.A., Belza, A.C.O., Melnicki, M.R., Montgomery, B.L., and Kerfeld, C.A. (2020). Cyanobacterial carboxysomes contain an unique Rubisco-activase-like protein. New Phytol. 225, 793-806.

Li, C., Salvucci, M.E., and Portis, A.R. (2005). Two Residues of Rubisco activase involved in recognition of the Rubisco substrate. J. Biol. Chem. 280, 24864-24869. 
Li, L.-A., Janet, L., Gibson, and Robert Tabita, F. (1993). The Rubisco activase ( $r c a)$ gene is located downstream from $r b c S$ in Anabaena sp. strain CA and is detected in other Anabaena/Nostoc strains. Plant Mol. Biol. 21, 753-764.

Li, L.A., Zianni, M.R., and Tabita, F.R. (1999). Inactivation of the monocistronic rca gene in Anabaena variabilis suggests a physiological ribulose bisphosphate carboxylase/oxygenase activase-like function in heterocystous cyanobacteria. Plant Mol. Biol. 40, 467-478.

Lin, M.T., Stone, W.D., Chaudhari, V., and Hanson, M.R. (2019). Enzyme kinetics of tobacco Rubisco expressed in Escherichia coli varies depending on the small subunit composition. BioRxiv 562223, doi: 10.1101/562223.

Long, B.M., Rae, B.D., Rolland, V., Forster, B., and Price, G.D. (2016). Cyanobacterial $\mathrm{CO}_{2}-$ concentrating mechanism components: function and prospects for plant metabolic engineering. Curr. Opin. Plant Biol. 31, 1-8.

Long, B.M., Tucker, L., Badger, M.R., and Price, G.D. (2010). Functional cyanobacterial betacarboxysomes have an absolute requirement for both long and short forms of the CcmM protein. Plant Physiol. 153, 285-293.

Mastronarde, D.N. (2005). Automated electron microscope tomography using robust prediction of specimen movements. J. Struct. Biol. 152, 36-51.

Matsumura, H., Mizohata, E., Ishida, H., Kogami, A., Ueno, T., Makino, A., Inoue, T., Yokota, A., Mae, T., and Kai, Y. (2012). Crystal structure of rice Rubisco and implications for activation induced by positive effectors NADPH and 6-phosphogluconate. J. Mol. Biol. 422, 75-86.

Mueller-Cajar, O. (2017). The diverse AAA+ machines that repair inhibited Rubisco active sites. Front. Mol. Biosci. 4, 31. doi: 10.3389/fmolb.2017.00031.

Mueller-Cajar, O., Stotz, M., Wendler, P., Hartl, F.U., Bracher, A., and Hayer-Hartl, M. (2011). Structure and function of the AAA+ protein CbbX, a red-type Rubisco activase. Nature 479, 194199.

Murshudov, G.N., Skubak, P., Lebedev, A.A., Pannu, N.S., Steiner, R.A., Nicholls, R.A., Winn, M.D., Long, F., and Vagin, A.A. (2011). REFMAC5 for the refinement of macromolecular crystal structures. Acta Crystallogr. D Biol. Crystallogr. 67, 355-367.

Newman, J., Branden, C.I., and Jones, T.A. (1993). Structure determination and refinement of ribulose 1,5-bisphosphate carboxylase/oxygenase from Synechococcus PCC6301. Acta Crystallogr. D Biol. Crystallogr. 49, 548-560.

Olivares, A.O., Baker, T.A., and Sauer, R.T. (2016). Mechanistic insights into bacterial AAA+ proteases and protein-remodelling machines. Nat. Rev .Microbiol. 14, 33-44. 
Ott, C.M., Smith, B.D., Portis, A.R., Jr., and Spreitzer, R.J. (2000). Activase region on chloroplast ribulose-1,5-bisphosphate carboxylase/oxygenase. Nonconservative substitution in the large subunit alters species specificity of protein interaction. J. Biol. Chem. 275, 2624126244.

Panjikar, S., Parthasarathy, V., Lamzin, V.S., Weiss, M.S., and Tucker, P.A. (2005). Autorickshaw: an automated crystal structure determination platform as an efficient tool for the validation of an X-ray diffraction experiment. Acta Crystallogr. D Biol. Crystallogr. 61, 449-457.

Pape, T., and Schneider, T.R. (2004). HKL2MAP: a graphical user interface for phasing with SHELX programs. J. Appl. Cryst. 37, 843-844.

Parry, M.A.J., Keys, A.J., Madgwick, P.J., Carmo-Silva, A.E., and Andralojc, P.J. (2008). Rubisco regulation: a role for inhibitors. J. Exp. Bot. 59, 1569-1580.

Pearce, F.G. (2006). Catalytic by-product formation and ligand binding by ribulose bisphosphate carboxylases from different phylogenies. Biochem. J. 399, 525-534.

Pena, K.L., Castel, S.E., de Araujo, C., Espie, G.S., and Kimber, M.S. (2010). Structural basis of the oxidative activation of the carboxysomal gamma-carbonic anhydrase, CcmM. Proc. Natl. Acad. Sci. U.S.A. 107, 2455-2460.

Pettersen, E.F., Goddard, T.D., Huang, C.C., Couch, G.S., Greenblatt, D.M., Meng, E.C., and Ferrin, T.E. (2004). UCSF Chimera - A visualization system for exploratory research and analysis. J. Comput. Chem. 25, 1605-1612.

Pierce, J., Tolbert, N.E., and Barker, R. (1980). Interaction of ribulose-bisphosphate carboxylase/oxygenase with transition-state analogs. Biochemistry 19, 934-942.

Portis, A.R., Li, C.S., Wang, D.F., and Salvucci, M.E. (2008). Regulation of Rubisco activase and its interaction with Rubisco. J. Exp. Bot. 59, 1597-1604.

Potterton, E., Briggs, P., Turkenburg, M., and Dodson, E. (2003). A graphical user interface to the CCP4 program suite. Acta Crystallogr. D Biol. Crystallogr. 59, 1131-1137.

Price, G.D., Coleman, J.R., and Badger, M.R. (1992). Association of carbonic anhydrase activity with carboxysomes isolated from the cyanobacterium Synechococcus PCC7942. Plant Physiol. 100, 784-793.

Price, G.D., Sültemeyer, D., Klughammer, B., Ludwig, M., and Badger, M.R. (1998). The functioning of the $\mathrm{CO}_{2}$ concentrating mechanism in several cyanobacterial strains: a review of general physiological characteristics, genes, proteins, and recent advances. Can. J. Bot. 76, 9731002 .

Puchades, C., Sandate, C.R., and Lander, G.C. (2020). The molecular principles governing the activity and functional diversity of AAA+ proteins. Nat. Rev. Mol. Cell Biol. 21, 43-58. 
Rae, B.D., Long, B.M., Badger, M.R., and Price, G.D. (2013). Functions, compositions, and evolution of the two types of carboxysomes: polyhedral microcompartments that facilitate $\mathrm{CO}_{2}$ fixation in cyanobacteria and some proteobacteria. Microbiol. Mol. Biol. Rev. 77, 357-379.

Rae, B.D., Long, B.M., Forster, B., Nguyen, N.D., Velanis, C.N., Atkinson, N., Hee, W.Y., Mukherjee, B., Price, G.D., and McCormick, A.J. (2017). Progress and challenges of engineering a biophysical $\mathrm{CO}_{2}$-concentrating mechanism into higher plants. J. Exp. Bot. 68, 3717-3737.

Ripstein, Z.A., Vahidi, S., Houry, W.A., Rubinstein, J.L., and Kay, L.E. (2020). A processive rotary mechanism couples substrate unfolding and proteolysis in the ClpXP degradation machinery. Elife 9, pii: e52158. doi: 10.7554/eLife.52158.

Rizo, A.N., Lin, J., Gates, S.N., Tse, E., Bart, S.M., Castellano, L.M., DiMaio, F., Shorter, J., and Southworth, D.R. (2019). Structural basis for substrate gripping and translocation by the ClpB AAA+ disaggregase. Nat. Commun. 10, 2393. doi: 10.1038/s41467-019-10150-y.

Robinson, S.P., and Portis, A.R., Jr. (1989). Adenosine triphosphate hydrolysis by purified Rubisco activase. Arch. Biochem. Biophys. 268, 93-99.

Rohou, A., and Grigorieff, N. (2015). CTFFIND4: Fast and accurate defocus estimation from electron micrographs. J. Struct. Biol. 192, 216-221.

Ryan, P., Forrester, T.J.B., Wroblewski, C., Kenney, T.M.G., Kitova, E.N., Klassen, J.S., and Kimber, M.S. (2019). The small RbcS-like domains of the $\beta$-carboxysome structural protein CcmM bind Rubisco at a site distinct from that binding the RbcS subunit. J. Biol. Chem. 294, 2593-2603.

Scheres, S.H. (2012). RELION: implementation of a Bayesian approach to cryo-EM structure determination. J. Struct. Biol. 180, 519-530.

Schindelin, J., Arganda-Carreras, I., Frise, E., Kaynig, V., Longair, M., Pietzsch, T., Preibisch, S., Rueden, C., Saalfeld, S., Schmid, B., et al. (2012). Fiji: an open-source platform for biologicalimage analysis. Nat. Methods 9, 676-682.

Servaites, J.C. (1985). Crystalline ribulose bisphosphate carboxylase/oxygenase of high integrity and catalytic activity from Nicotiana tabacum. Arch. Biochem. Biophys. 238, 154-160.

Sharwood, R.E. (2017). Engineering chloroplasts to improve Rubisco catalysis: prospects for translating improvements into food and fiber crops. New Phytol. 213, 494-510.

Sheldrick, G.M. (2010). Experimental phasing with SHELXC/D/E: combining chain tracing with density modification. Acta Crystallogr. D Biol. Crystallogr. 66, 479-485.

Shivhare, D., and Mueller-Cajar, O. (2017). In vitro characterization of thermostable CAM Rubisco activase reveals a Rubisco interacting surface loop. Plant Physiol. 174, 1505-1516. 
Shivhare, D., Ng, J., Tsai, Y.-C.C., and Mueller-Cajar, O. (2019). Probing the rice RubiscoRubisco activase interaction via subunit heterooligomerization. Proc. Natl. Acad. Sci. U.S.A. $116,24041-24048$.

Sievers, F., Wilm, A., Dineen, D., Gibson, T.J., Karplus, K., Li, W., Lopez, R., McWilliam, H., Remmert, M., Söding, J., et al. (2011). Fast, scalable generation of high-quality protein multiple sequence alignments using Clustal Omega. Mol. Syst. Biol. 7, 539. doi: 10.1038/msb.2011.75.

Singer, S.D., Soolanayakanahally, R.Y., Foroud, N.A., and Kroebel, R. (2019). Biotechnological strategies for improved photosynthesis in a future of elevated atmospheric $\mathrm{CO}_{2}$. Planta 251, 24. doi: 10.1007/s00425-019-03301-4.

Slattery, R.A., and Ort, D.R. (2019). Carbon assimilation in crops at high temperatures. Plant Cell Environ. 42, 2750-2758.

Stotz, M., Mueller-Cajar, O., Ciniawsky, S., Wendler, P., Hartl, F.U., Bracher, A., and HayerHartl, M. (2011). Structure of green-type Rubisco activase from tobacco. Nat. Struct. Mol. Biol. $18,1366-1370$.

Studier, F.W. (2005). Protein production by auto-induction in high density shaking cultures. Protein Expr. Purif. 41, 207-234.

Tabita, F.R., Satagopan, S., Hanson, T.E., Kreel, N.E., and Scott, S.S. (2008). Distinct form I, II, III, and IV Rubisco proteins from the three kingdoms of life provide clues about Rubisco evolution and structure/function relationships. J. Exp. Bot. 59, 1515-1524.

Terwilliger, T.C. (2000). Maximum-likelihood density modification. Acta Crystallogr. D Biol. Crystallogr. 56, 965-972.

Thévenaz, P., Ruttimann, U.E., and Unser, M. (1998). A pyramid approach to subpixel registration based on intensity. IEEE Trans Image Process 7, 27-41.

Turmo, A., Gonzalez-Esquer, C.R., and Kerfeld, C.A. (2017). Carboxysomes: metabolic modules for $\mathrm{CO}_{2}$ fixation. FEMS Microbiol. Lett. 364. doi: 10.1093/femsle/fnx176.

Twomey, E.C., Ji, Z., Wales, T.E., Bodnar, N.O., Ficarro, S.B., Marto, J.A., Engen, J.R., and Rapoport, T.A. (2019). Substrate processing by the Cdc48 ATPase complex is initiated by ubiquitin unfolding. Science 365, pii: eaax 1033. doi: 10.1126/science.aax1033.

Vagin, A.A., and Isupov, M.N. (2001). Spherically averaged phased translation function and its application to the search for molecules and fragments in electron-density maps. Acta Crystallogr. D Biol. Crystallogr. 57, 1451-1456. 
Wachter, R.M., Salvucci, M.E., Carmo-Silva, A.E., Barta, C., Genkov, T., and Spreitzer, R.J. (2013). Activation of interspecies-hybrid Rubisco enzymes to assess different models for the Rubisco-Rubisco activase interaction. Photosynth. Res. 117, 557-566.

Wang, H., Yan, X., Aigner, H., Bracher, A., Nguyen, N.D., Hee, W.Y., Long, B.M., Price, G.D., Hartl, F.U., and Hayer-Hartl, M. (2019). Rubisco condensate formation by CcmM in $\beta$ carboxysome biogenesis. Nature 566, 131-135.

Wang, L., Myasnikov, A., Pan, X., and Walter, P. (2020). Structure of the AAA protein Msp1 reveals mechanism of mislocalized membrane protein extraction. Elife 9, pii: e54031. doi: 10.7554/eLife.54031.

Wang, Z.Y., and Portis, A.R. (1992). Dissociation of ribulose-1,5-bisphosphate bound to ribulose-1,5-bisphosphate carboxylase/oxygenase and its enhancement by ribulose-1,5bisphosphate carboxylase/oxygenase activase-mediated hydrolysis of ATP. Plant Physiol. 99, 1348-1353.

Waterhouse, A., Bertoni, M., Bienert, S., Studer, G., Tauriello, G., Gumienny, R., Heer, F.T., de Beer, T.A.P., Rempfer, C., Bordoli, L., et al. (2018). SWISS-MODEL: homology modelling of protein structures and complexes. Nucleic Acids Res. 46, W296-W303.

Wickham, H., Averick, M., Bryan, J., Chang, W., McGowan, L.D., François, R., Grolemund, G., Hayes, A., Henry, L., Hester, J., et al. (2019). Welcome to the tidyverse. J. Open Source Softw. 4, 1686. doi: 10.21105/joss.01686.

Wilson, R.H., Thieulin-Pardo, G., Hartl, F.U., and Hayer-Hartl, M. (2019). Improved recombinant expression and purification of functional plant Rubisco. FEBS Lett. 593, 611-621.

Wyatt, P.J. (1993). Light scattering and the absolute characterization of macromolecules. Anal. Chim. Acta. 272, 1-40.

Zheng, S.Q., Palovcak, E., Armache, J.P., Verba, K.A., Cheng, Y., and Agard, D.A. (2017). MotionCor2: anisotropic correction of beam-induced motion for improved cryo-electron microscopy. Nat. Methods 14, 331-332. 
A

Prokaryotic Rca (CbbQ) from H. neopolitanus (HnRca 30 kDa)

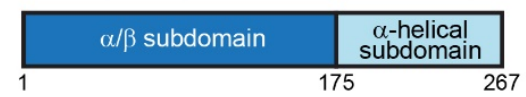

Prokaryotic Rca from $R$. sphaeroides (RsRca $\sim 35 \mathrm{kDa})$

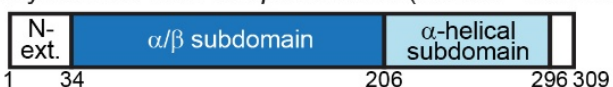

Eukaryotic Rca from N. tabacum (NtRca $\sim 42 \mathrm{kDa})$

\begin{tabular}{|c|c|c|c|}
\hline $\begin{array}{c}\mathrm{N}- \\
\text { domain }\end{array}$ & $\alpha / \beta$ subdomain & $\begin{array}{c}\alpha \text {-helical } \\
\text { subdomain }\end{array}$ & $\begin{array}{c}\mathrm{C}- \\
\text { ext. }\end{array}$ \\
\hline 1
\end{tabular}

Prokaryotic 'Rca-like' from Nostoc sp. PCC 7120 (NosRca 47 kDa)

\begin{tabular}{|c|c|c|c|}
\hline$\alpha / \beta$ subdomain & $\begin{array}{c}\alpha \text {-helical } \\
\text { subdomain }\end{array}$ & & SSUL \\
\hline
\end{tabular}

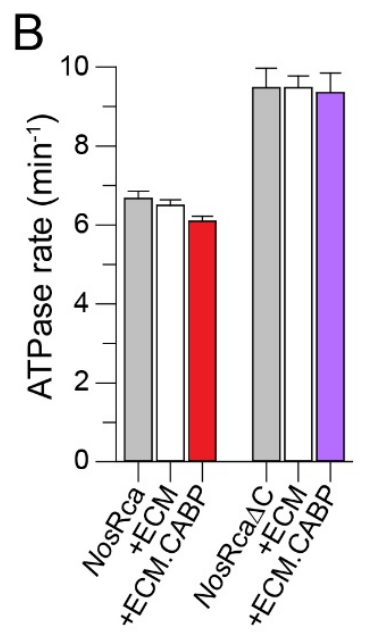

C

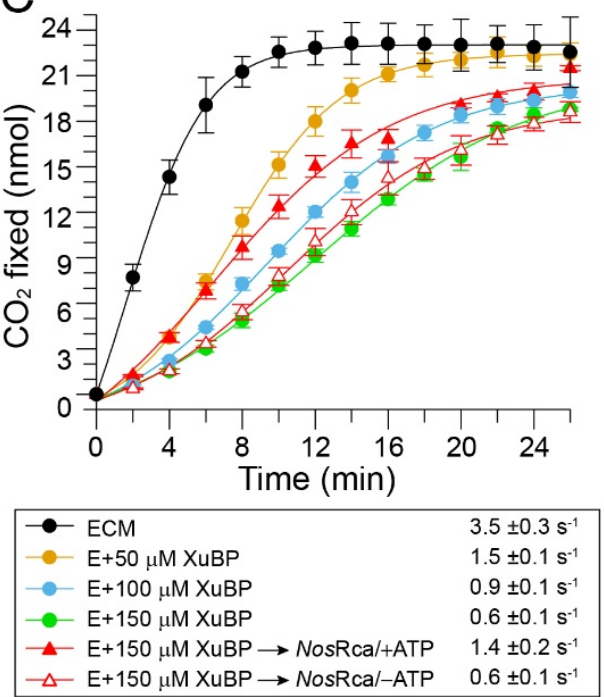

$\mathrm{AAA}+$ core

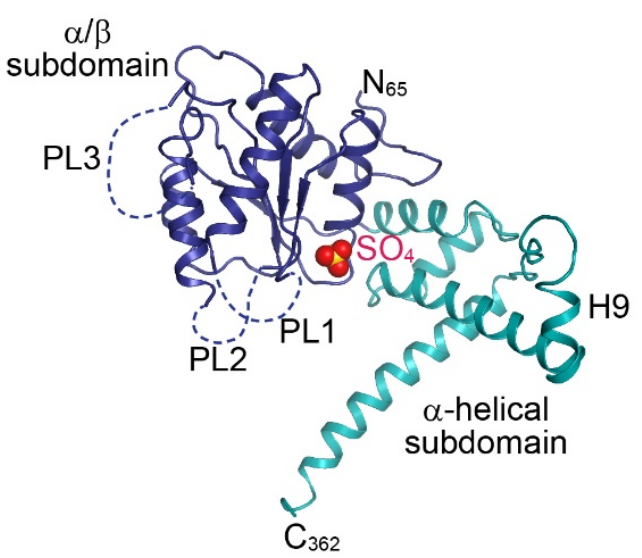

D

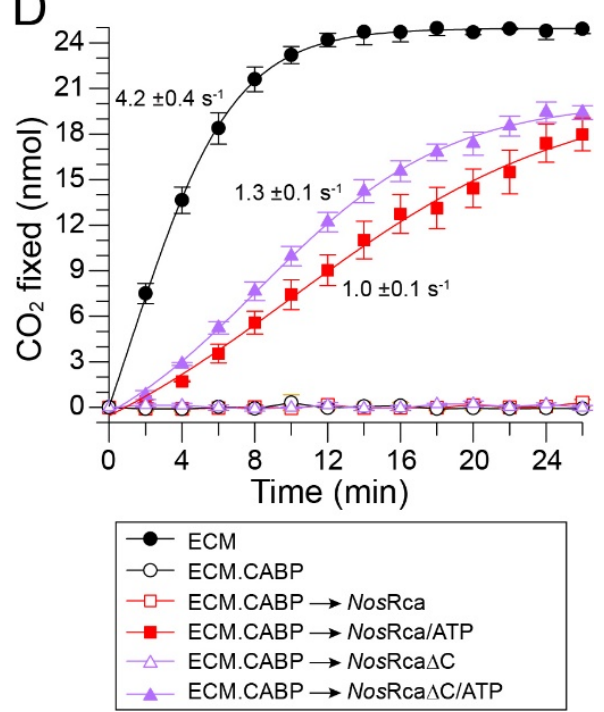

Figure 1. Rubisco Activase Function of NosRca

(A) Left: Schematic representation of the domain structures of Rca proteins across phylogenetic groups. CbbQ from the chemoautotroph Halothiobacillus neapolitanus (HnRca), CbbX from $R$. sphaeroides (RsRca; prokayotic, red-type), Rca from $N$. tabacum ( $N t$ Rca; eukaryotic, green-type) and cyanobacterial 'Rca-like' (NosRca; prokaryotic, green-type) from Nostoc sp. PCC 7120. Right: Ribbon representation of Rca from A. thaliana (AtRca; PDB: 4W5W) (Hasse et al., 2015). $\alpha / \beta$ and $\alpha$-helical subdomains as well as the pore-loops PL1, PL2 and PL3, and helix H9 are indicated. A bound sulfate ion bound to the nucleotide binding pocket is represented as spacefilling model. (B) ATPase rates of NosRca and NosRca $\Delta \mathrm{C}$ in presence or absence of active (ECM) or inhibited (ECM.CABP) NosRubisco. ATPase rates were measured at $0.5 \mu \mathrm{M}$ Rca (hexamer) and $0.25 \mu \mathrm{M}$ NosRubisco (hexadecamer) as indicated. Bars represent averages with SD from at least three independent replicates. See STAR Methods for details. (C) NosRca functions as a Rubisco activase. $\mathrm{CO}_{2}$ fixation assays were performed with Rubisco enzyme (E, $0.25 \mu \mathrm{M})$ in presence of increasing concentrations of $\operatorname{XuBP}(50,100$ and $150 \mu \mathrm{M})$, and in presence or absence of $0.5 \mu \mathrm{M}$ Rca (hexamer) and $3 \mathrm{mM}$ ATP. The active NosRubisco (ECM) was used as control. Approximate rates of $\mathrm{CO}_{2}$ fixation were determined from the linear parts of 
bioRxiv preprint doi: https://doi.org/10.1101/2020.05.16.099382; this version posted May 16, 2020. The copyright holder for this preprint (which

was not certified by peer review) is the author/funder, who has granted bioRxiv a license to display the preprint in perpetuity. It is made available under aCC-BY-NC-ND 4.0 International license.

the curves. Data represent SD from at least three independent replicates. (D) Reactivation of CABP inhibited NosRubisco (ECM.CABP) by NosRca or NosRca $\Delta$ C. $\mathrm{CO}_{2}$ fixation assays were performed as in (C) with ECM.CABP in the presence or absence of ATP as indicated. Bars represent averages with $\mathrm{SD}$ of at least three independent replicates. 
A Chain A

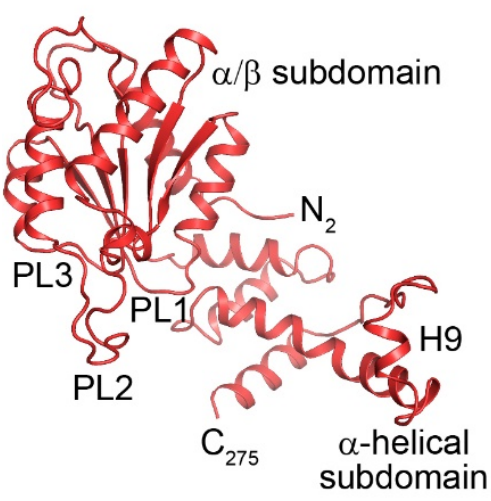

B Chain B

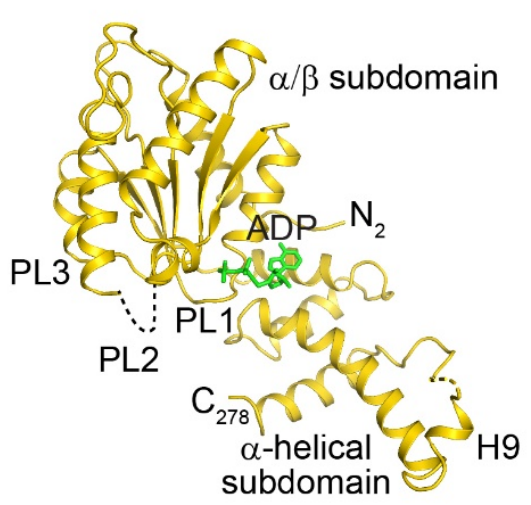

C

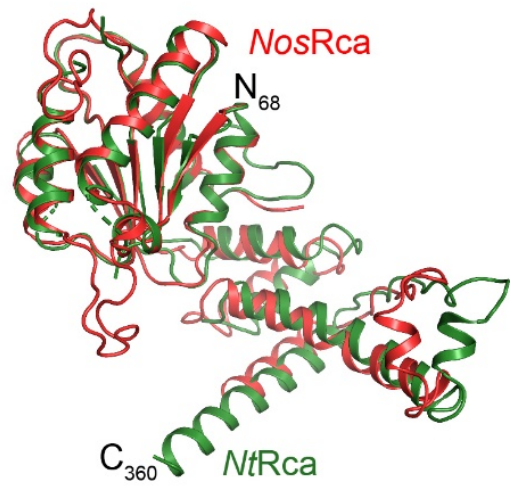

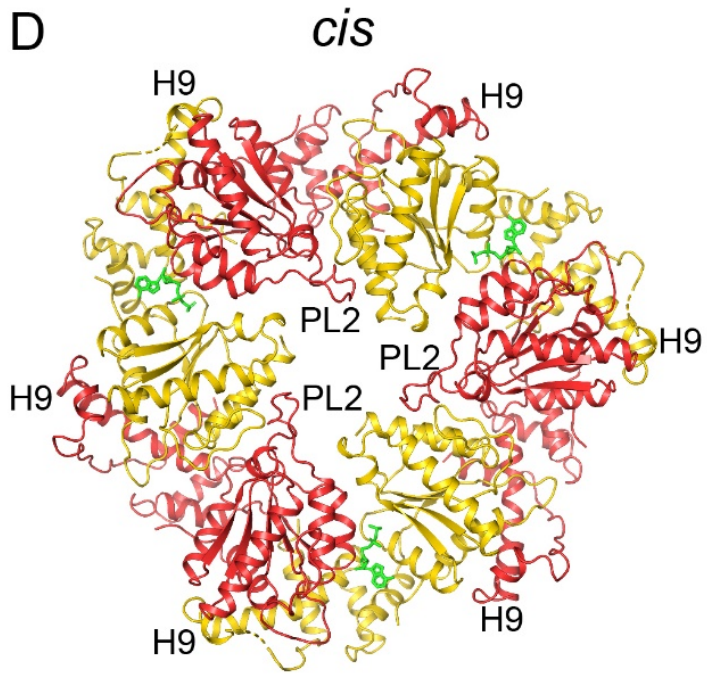
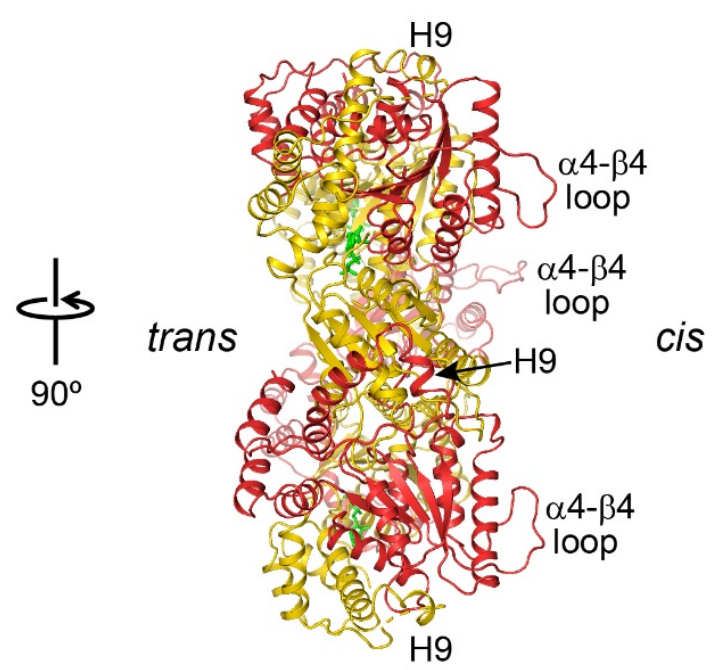

Figure 2. Crystal Structure of NosRca $\Delta \mathrm{C}$

(A) and (B) Ribbon representations of the two crystallographically independent chains of NosRca $\Delta \mathrm{C}$ in red and yellow, respectively. The subdomain structure as well as locations of helix H9 and pore-loops PL1, PL2 and PL3 are indicated. ADP is represented as a wire-frame model in green. Chain termini are indicated. (C) Superposition of chain A (no nucleotide bound) of the asymmetric unit of $N o s$ Rca $\Delta$ C with $N t$ Rca (PDB: 3T15) (Stotz et al., 2011). Chain A of Nos Rca $\Delta \mathrm{C}$ and $N t \mathrm{Rca}$ are shown as ribbons in red and green, respectively. (D) NosRca $\Delta \mathrm{C}$ hexamer. The hexamer was generated by applying the crystallographic three-fold rotational symmetry. The locations of helix H9 and pore-loop PL2 and loop $\alpha 4-\beta 4$ are indicated. Chain A, red; chain B, yellow. 
A

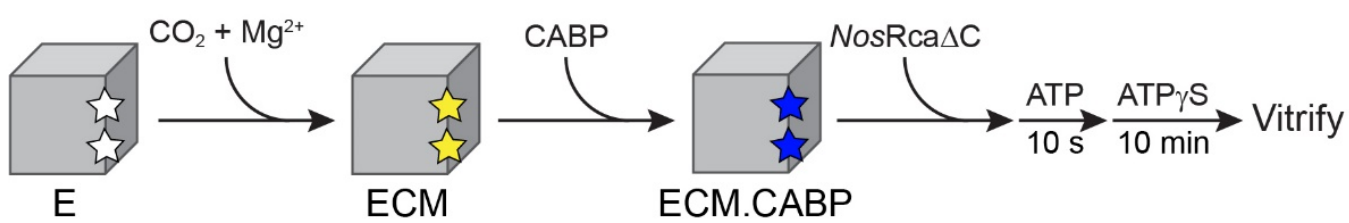

B

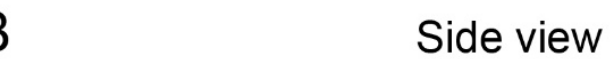

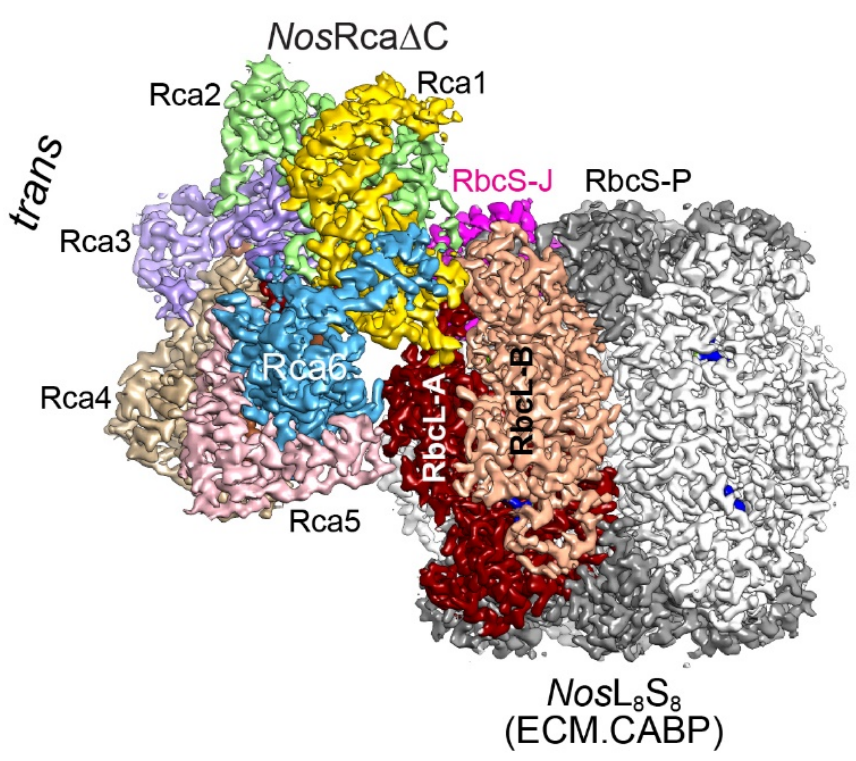

C

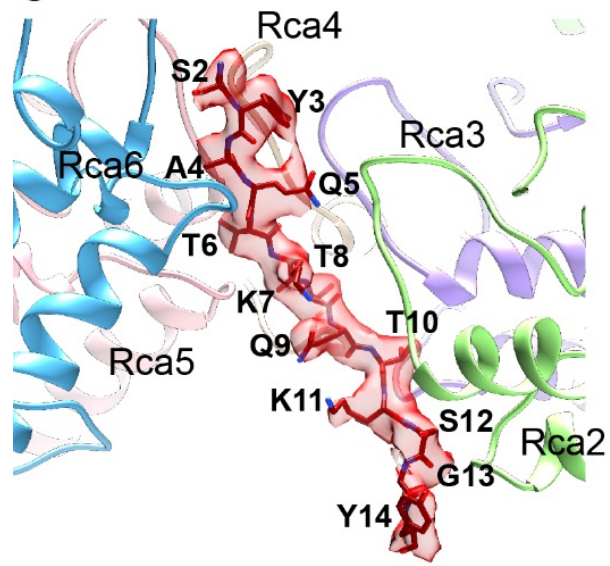

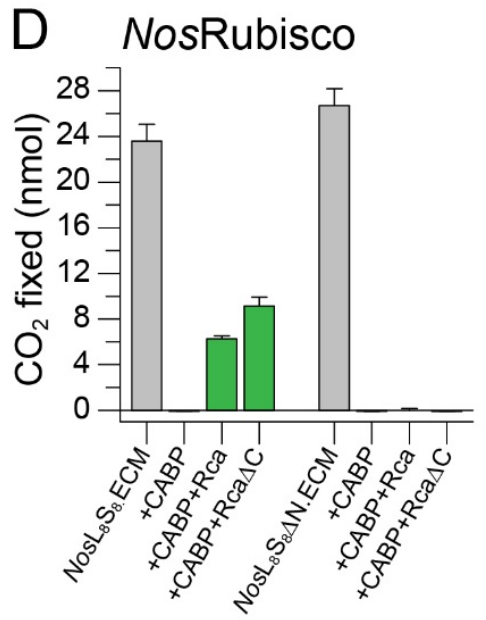

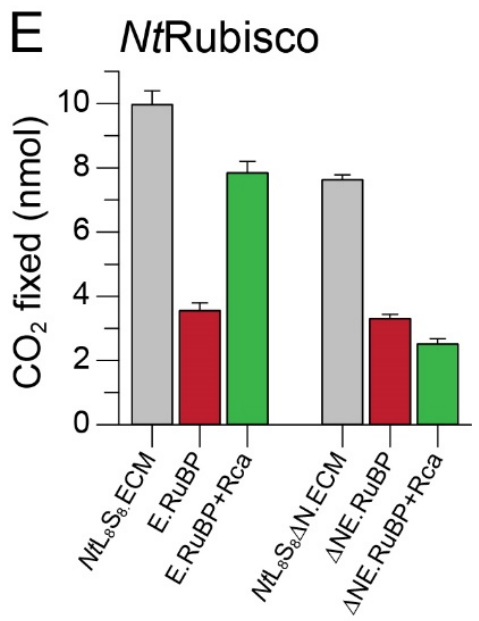

Figure 3. Cryo-EM Structure of the NosRca $\triangle \mathrm{C}$ :Rubisco Complex

(A) Schematic representation of preparation of the NosRca $\Delta$ C:Rubisco complex analyzed by cryo-EM. (B) Cryo-EM reconstruction of the NosRca $\Delta$ C:Rubisco complex in two perpendicular views. The subunits in the hexameric NosRca ring are labeled Rcal to Rca6, with Rcal (yellow) closest to and Rca6 (blue) farthest from Rubisco, respectively. The anti-parallel RbcL dimer subunits RbcL-A and RbcL-B as well as the RbcS subunits RbcS-J and RbcS-P in contact with NosRca are indicated. (C) Cryo-EM density of the N-terminal RbcL-A peptide bound inside the 
NosRca hexamer pore, interacting with Rca subunits (ribbon representation) Rca2 to Rca6. Amino acid residues of 2 to 14 of the bound peptide are indicated in single letter code. (D) Reactivation of NosRubisco by NosRca depends on the N-terminus of RbcL. $\mathrm{CO}_{2}$ fixation assays were performed as in Figure 1C with CABP-inhibited NosRubisco $\left(N_{o s} \mathrm{~L}_{8} \mathrm{~S}_{8}\right)$ and $N_{o s} \mathrm{~L}_{8} \mathrm{~S}_{8} \Delta \mathrm{N}$ in the presence or absence of NosRca or NosRca $\Delta \mathrm{C}$ and ATP for $8 \mathrm{~min}$. Bars represent averages with SD from at least three independent experiments. (E) Reactivation of $N t$ Rubisco by $N t$ Rca depends on the N-terminus of RbcL. $\mathrm{CO}_{2}$ fixation assays were performed with RuBP-inhibited $N t \mathrm{~L}_{8} \mathrm{~S}_{8}(\mathrm{E} . \mathrm{RuBP})$ and $N t \mathrm{~L}_{8} \mathrm{~S}_{8} \Delta \mathrm{N}(\Delta \mathrm{NE} . \mathrm{RuBP})(0.25 \mu \mathrm{M}$ active sites) in the presence or absence of $N t$ Rca $(0.5 \mu \mathrm{M}$ hexamer) and ATP for $8 \mathrm{~min}$. Bars represent averages with SD from at least three independent experiments. See STAR Methods for details. 
A

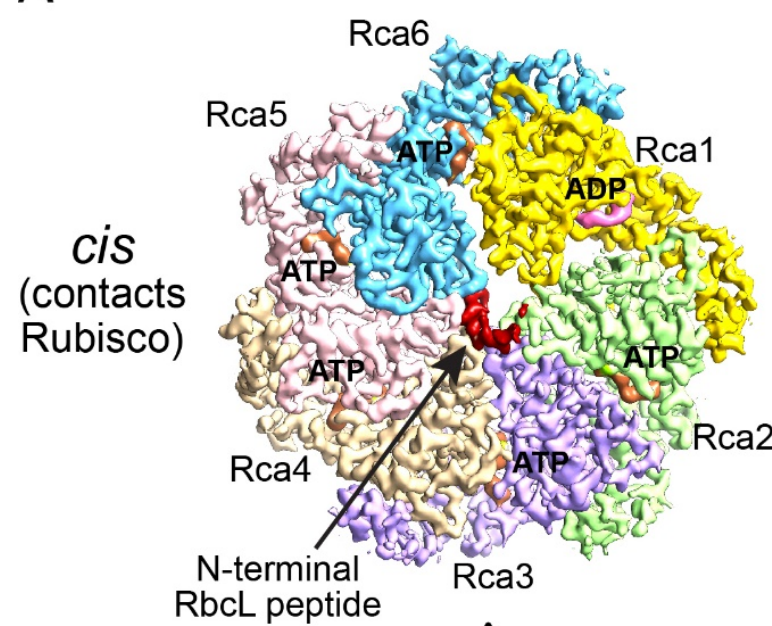

RbcL peptide

Side

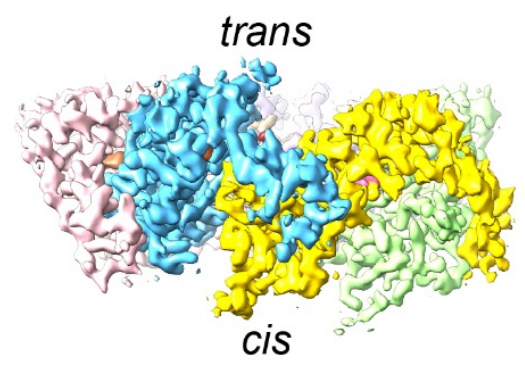

trans

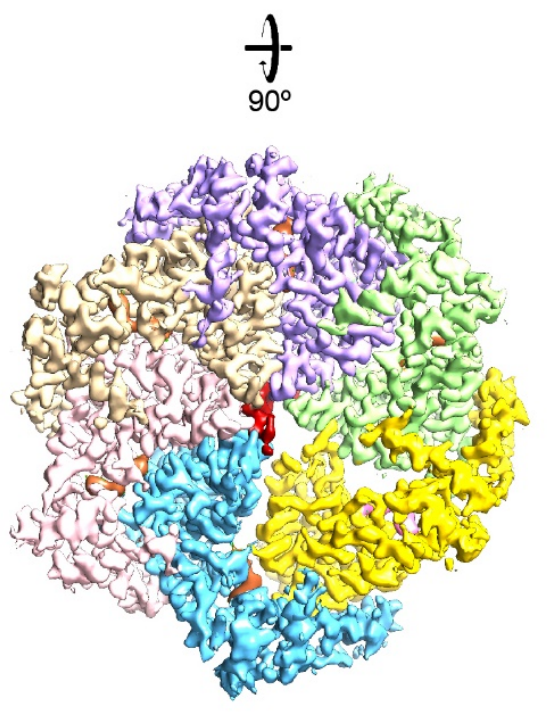

B
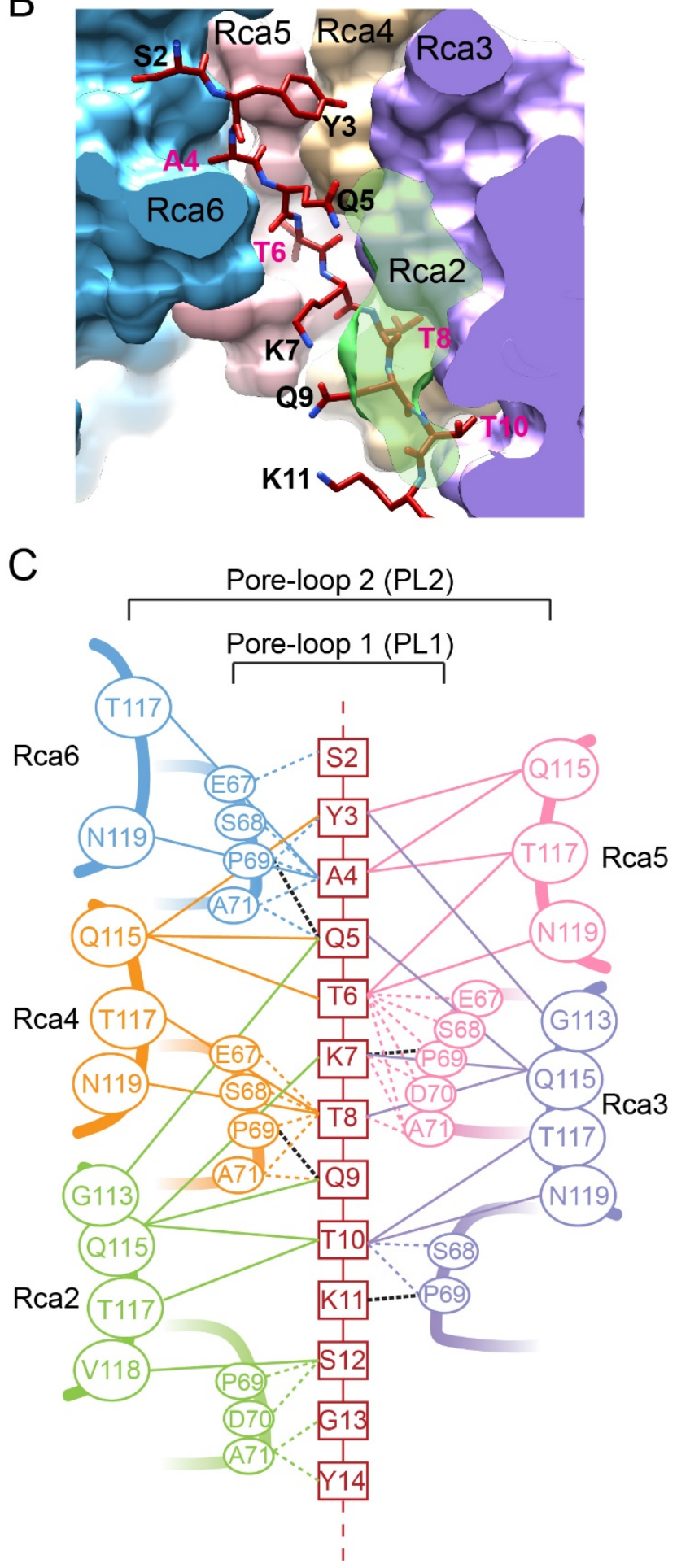

Figure 4. NosRca in a Helical 'Split-washer' Conformation Interacts via its Central Pore with the N-terminal RbcL Peptide

(A) Cryo-EM density map of NosRca $\Delta \mathrm{C}$ in the $N o s \mathrm{Rca} \Delta \mathrm{C}$ :Rubisco complex in cis (surface in contact with Rubisco) and trans view. ADP in pink, ATP/ATP $\gamma \mathrm{S}$ in sienna and the bound N- 
terminal peptide from RbcL-A in red. NosRca subunits are color coded as in Figure 3B. (B) Cavities in the NosRca central pore. Small cavities formed between pore-loops PL1 and PL2 from adjacent Rca subunits (Rca2 to Rca6) accommodate the side-chains of the alternating small amino acids of the RbcL N-terminal peptide. The bulky side-chains point to the solvent channel. The NosRca central pore is in surface representation and the RbcL N-terminal peptide in stick representation. (C) Interactions (van der Waals and hydrogen-bond) between residues of the RbcL N-terminal peptide and the staggered pore-loops PL1 (dotted colored lines) and PL2 (solid colored lines) of the Rca subunits. The distance cutoff was $4 \AA$. Putative hydrogen-bonds to the peptide backbone are indicated by black dotted lines. 

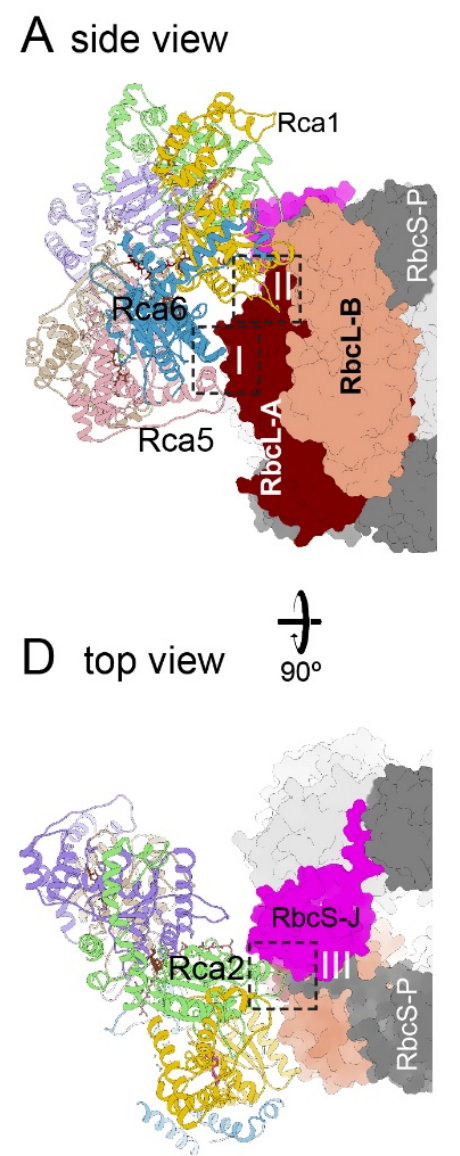

G

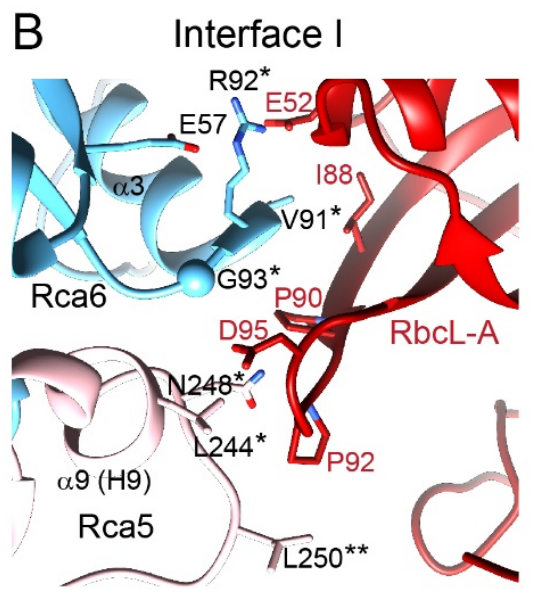

E Interface III

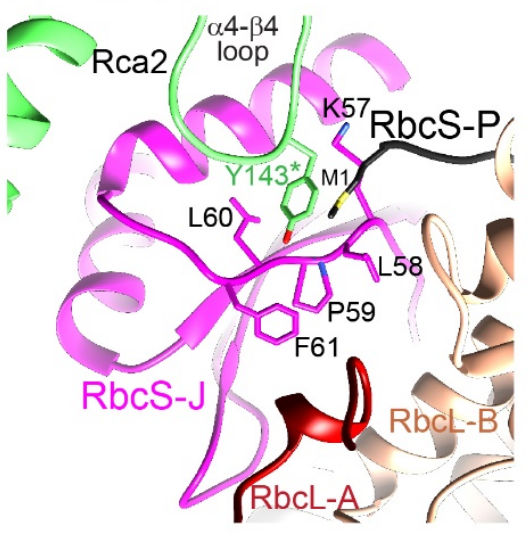

$\mathrm{F}$
C Interface II

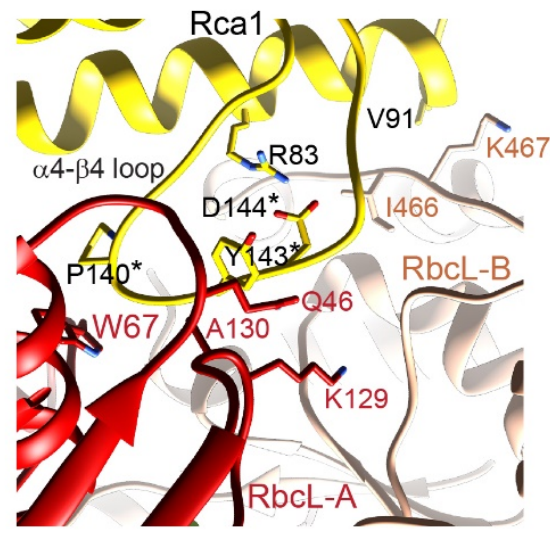

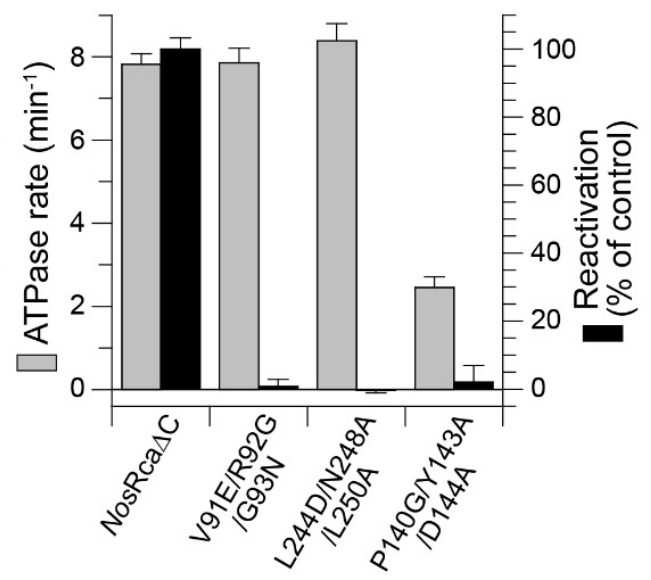

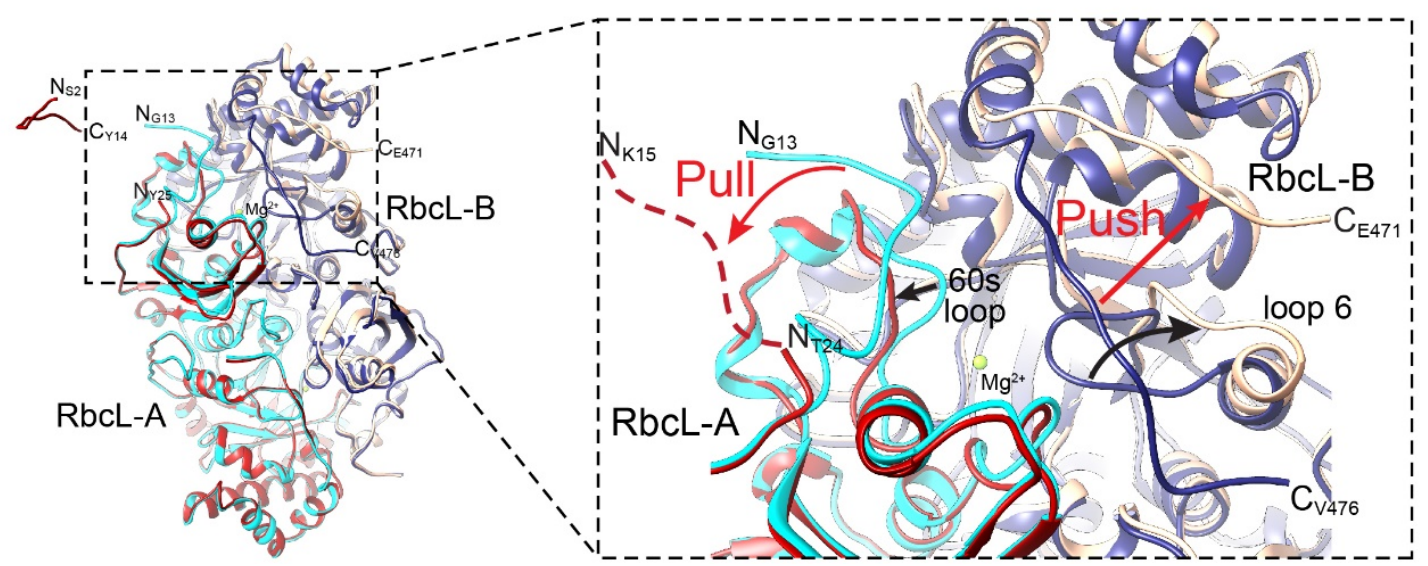

Figure 5. Structural Details of the NosRca:Rubisco Interaction

(A-E) Interfaces of the NosRca $\Delta$ C:Rubisco complex. Overview of the complex, highlighting interfaces I and II with NosRca in ribbon representation and Rubisco in surface representation (A). Interacting amino acid residues in interface I (B) and interface II (C). In interface I, Rca5 and Rca6 (subunits color-coded as in Figure 3B) interact with RbcL-A. Helix $\alpha 9$ (H9) in Rca5 and helix $\alpha 3$ in Rca6 are indicated. In interface II, the $\alpha 4-\beta 4$ loop of Rca1 makes contacts with 
RbcL-A. Overview of the complex highlighting interface III (D). In interface III, the residue Tyr143 of the $\alpha 4-\beta 4$ loop of Rca 2 makes contacts with RbcS-J and RbcS-P (E). Sidechains of contact residues are shown in stick representation. *, residue analyzed by mutation. $(\mathrm{F})$ Mutational analysis of NosRca interface residues. ATPase rates of NosRca $\Delta \mathrm{C}$ and mutants (NosRca $\Delta$ C V91E/R92G/G93N, NosRca $\Delta$ C P140G/Y143A/D14A, NosRca $\Delta \mathrm{C}$

L244D/N248A/L250A) and reactivation of CABP-inhibited NosRubisco were measured as in Figures $1 \mathrm{~B}$ and 3D, respectively. ATPase rates were measured in the absence of Rubisco at 20 $\mathrm{mM} \mathrm{KCl}$ (see STAR Methods). $\mathrm{CO}_{2}$ fixation was measured for $8 \mathrm{~min}$ and set to $100 \%$ for wildtype NosRca $\Delta$ C. Error bars represent SD of at least three independent experiments. (G) Structural remodeling of the Rubisco substrate binding pocket. Super-position of the binding pocket, formed at the interface between the $\mathrm{N}$ - and C-terminal domains of the anti-parallel RbcL subunits, in the closed (RbcL-A light blue; RbcL-B dark blue) and the NosRca-engaged open state (RbcL-A red; RbcL-B peach). The remodeled regions, including the 60s loop of RbcL-A and loop 6 of RbcL-B as well as the pull and push actions of NosRca are indicated. 
A

Nostoc sp. PCC 7120 (NosRca)

\begin{tabular}{|l|c|c|c|}
\hline$\alpha / \beta$ subdomain & $\begin{array}{c}\alpha \text {-helical } \\
\text { subdomain }\end{array}$ & & SSUL \\
\hline 1
\end{tabular}

Nostoc sp PCC 7120 (scaffolding protein CcmM) NosM58

\begin{tabular}{|c|c|c|c|}
\hline $\mathrm{CA}$ & SSUL1 & SSUL2 & SSUL3 \\
\hline \multicolumn{4}{|c|}{$\frac{1}{313}$} \\
\hline NosM35 & SSUL1 & SSUL2 & SSUL3 \\
\hline
\end{tabular}

C

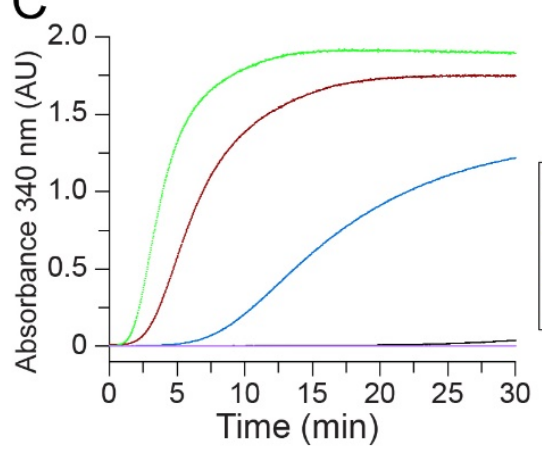

E reNosRca/ATP $\gamma$ S Rubisco
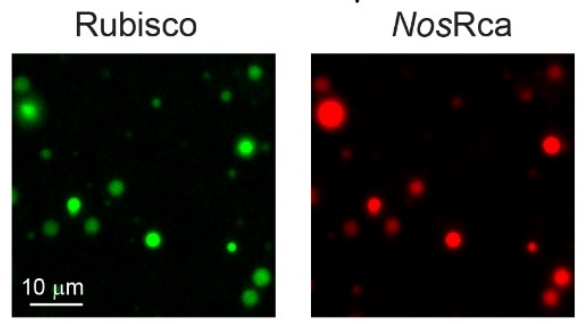

G

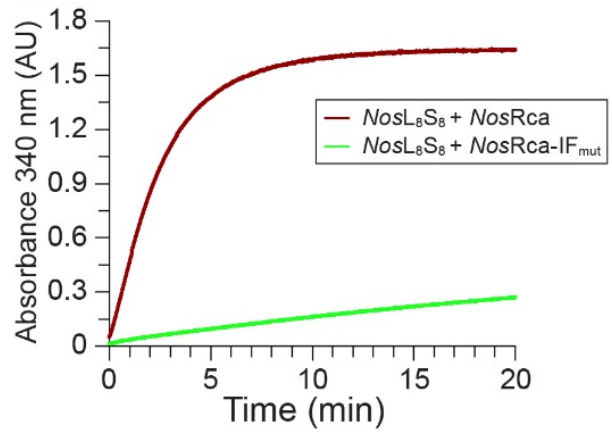

B
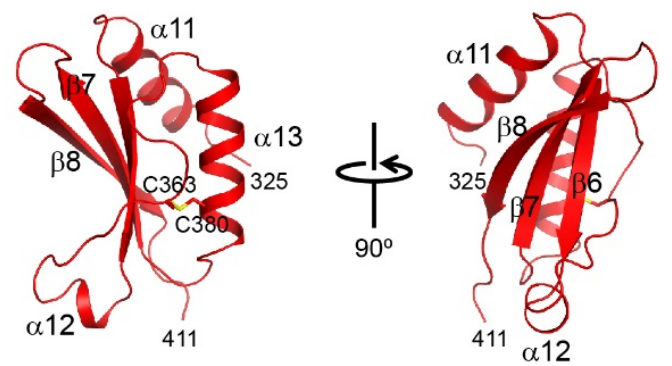

D

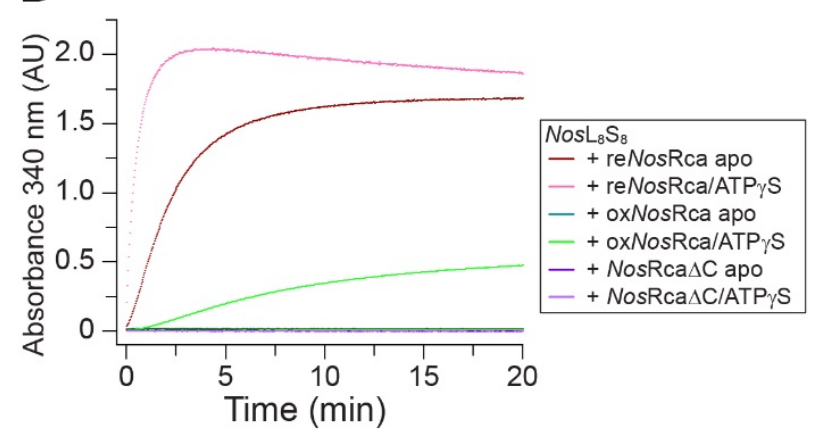

F NosRca $\Delta$ C/ATP $\gamma$ S

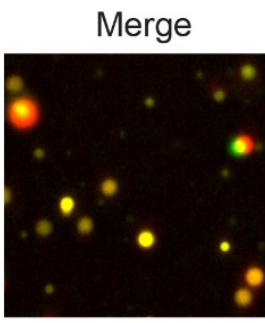

Rubisco

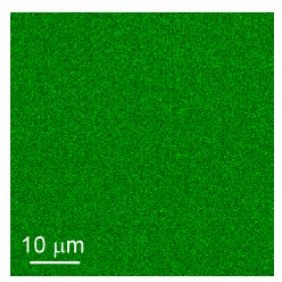

$\mathrm{H}$ reNosRca-IF ${ }_{\text {mut }}$

Rubisco
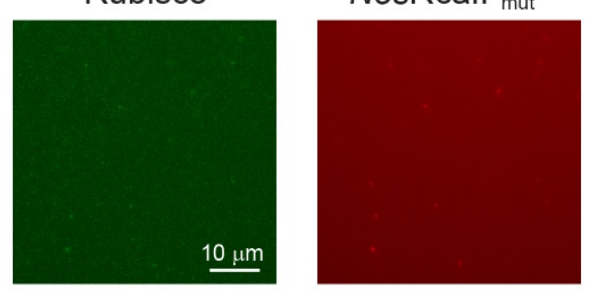

NosRca $\Delta C$

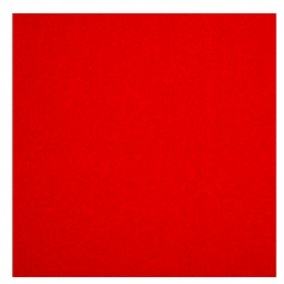

Merge

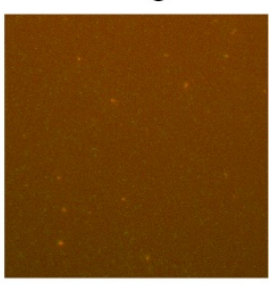

Figure 6. Cooperation of SSUL Domains and AAA+ Core in Carboxysome Organization (A) Schematic representation of the domain structures of NosRca and Nos CcmM (full length Nos M58 and the shorter isoform NosM35). CA, carbonic anhydrase domain. (B) Crystal structure of the SSUL domain of NosRca in ribbon representation. Chain termini, secondary structure elements and the disulfide bond (Cys360-Cys380) are indicated. (C) Formation of NosRca-Rubisco condensate mediated by the SSUL domains analyzed by turbidity assay. Data 
shown are for Rubisco $\left(N o s \mathrm{~L}_{8} \mathrm{~S}_{8} ; 0.25 \mu \mathrm{M}\right)$ with increasing concentrations of reduced Nos Rca from $0.125 \mu \mathrm{M}$ to $1 \mu \mathrm{M}$ or with $\operatorname{Nos} \operatorname{Rca} \Delta \mathrm{C}(1 \mu \mathrm{M})$. Representative results from at least three independent experiments are shown. (D) Nucleotide and redox-dependence of NosRca-Rubisco condensate formation. Rubisco $(0.25 \mu \mathrm{M})$ was combined with reduced (re) or oxidized (ox) NosRca $(0.5 \mu \mathrm{M})$, or NosRca $\Delta \mathrm{C}(0.5 \mu \mathrm{M})$, in the presence or absence of ATP $\gamma \mathrm{S}(2 \mathrm{mM})$. Condensate formation was measured by turbidity assay as in (C). Representative results from at least three independent experiments are shown. (E and F) Formation of NosRca-Rubisco condensate in the presence of $\mathrm{ATP} \gamma \mathrm{S}$ as observed by fluorescence microscopy. Fluorophore labeled proteins (A405-NosRca, A405-NosRca $\Delta \mathrm{C}$ and A532-NosRubisco) were mixed 1:10 with the respective unlabeled proteins and used at total concentrations of reduced NosRca and Rubisco of $0.5 \mu \mathrm{M}$ and $0.25 \mu \mathrm{M}$, respectively. Rubisco was mixed with either reduced Nos Rca (E) or NosRca $\Delta \mathrm{C}$ (F) in the presence of $2 \mathrm{mM}$ ATP $\gamma$ S. See STAR Methods for details. (G) NosRca-Rubisco condensate formation analyzed as in (D). Rubisco $(0.25 \mu \mathrm{M})$ was combined with reduced $N o s$ Rca or $N o s \mathrm{Rca}_{-} \mathrm{IF}_{\mathrm{mut}}(0.5 \mu \mathrm{M})$. Representative results from at least three independent experiments are shown. $(\mathrm{H})$ Inability of Nos Rca-IF mut to form a condensate with Rubisco, observed by fluorescence microscopy. Fluorophore labeled A405-NosRca-IF mut and A532-NosRubisco were used as described in (E). 
A NosRca:Rubisco

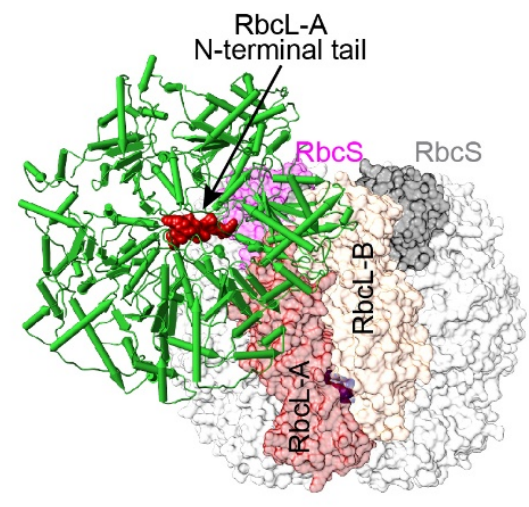

C

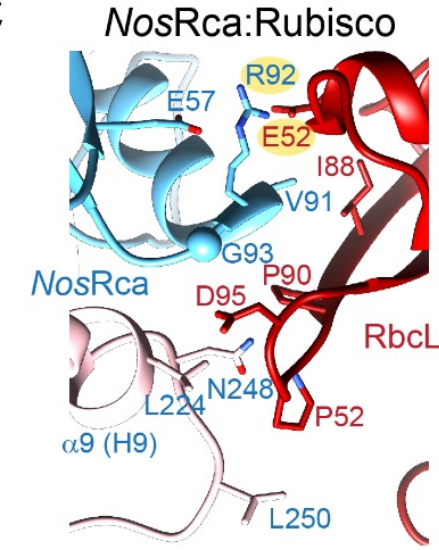

$\mathrm{D}$
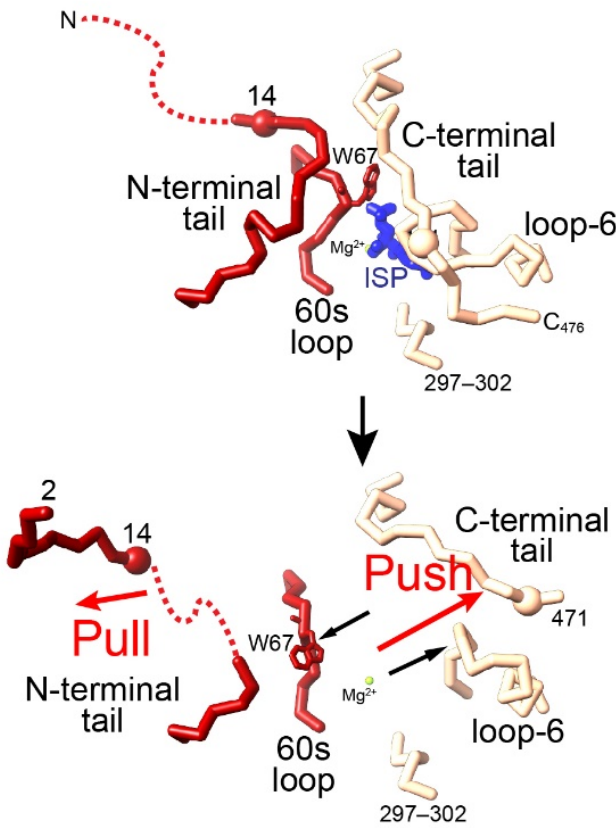

B
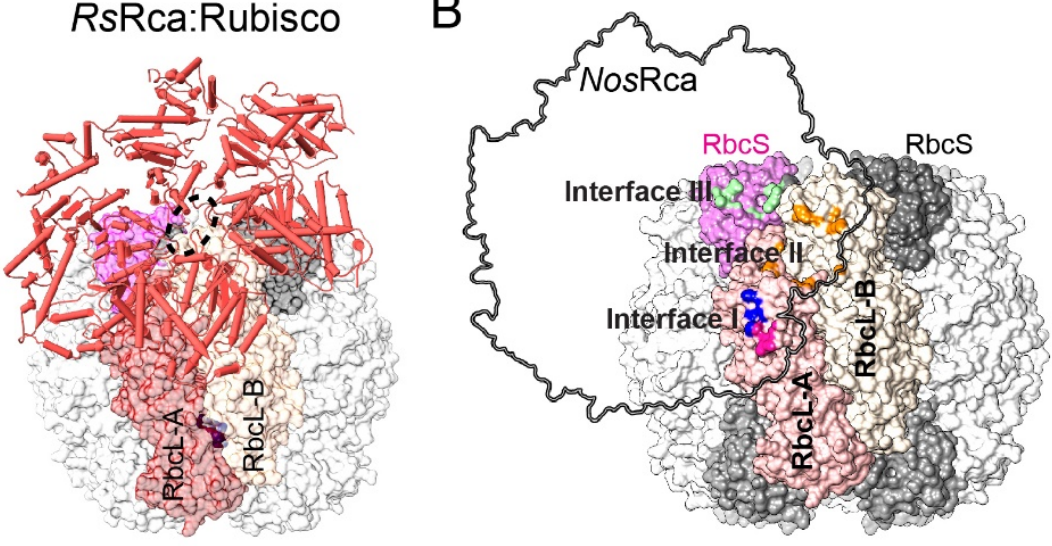

AtRca:Rubisco

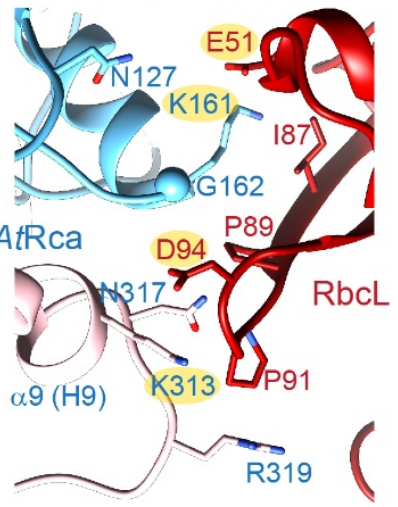

NtRca:Rubisco

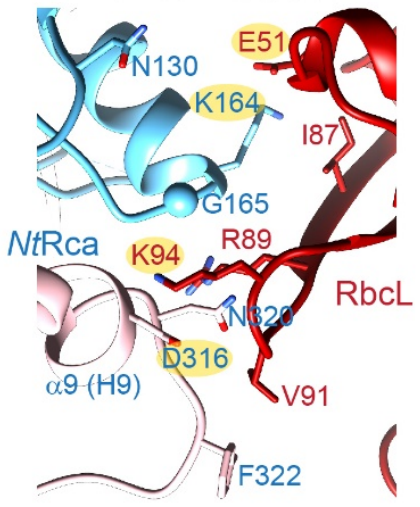

E

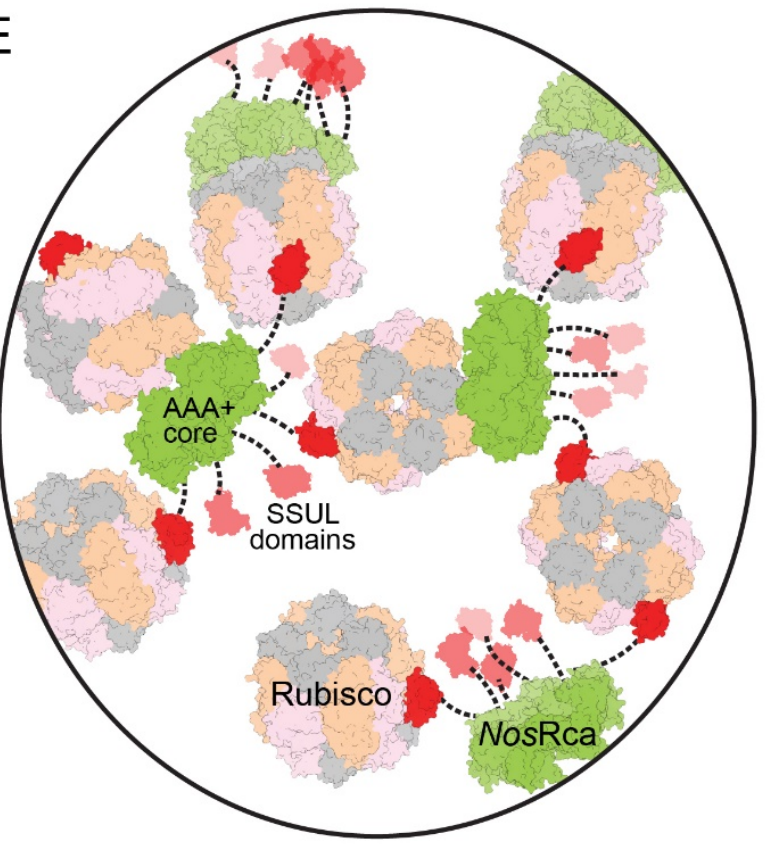


Figure 7. Mechanism of NosRca in Rubisco Reactivation and Carboxysome Organization (A) Comparison of the positioning of NosRca (left) and RsRca (right) in complex with their cognate Rubisco for engagement of the $\mathrm{N}$-terminus and $\mathrm{C}$-terminus of RbcL, respectively. In both structures Rubisco is shown in surface representation in the same orientation.The antiparallel RbcL dimer and the RbcS subunits involved in the interaction with Rca are indicated. RsRca:Rubisco model, EMDB: EMD-3701/PDB: 3ZUH and PDB: 5NV3. (B) Overview of the surface areas on NosRubisco interacting with NosRca via interfaces I (red and dark blue), II (orange) and III (light blue). NosRca is shown as an outline. NosRubisco colors as in (A). (C) Structural models for interface I in the AtRca:AtRubisco (middle) and NtRca:NtRubisco (right) complexes. On the left, interface I in the NosRca:NosRubisco complex is shown. The protein backbones are shown in ribbon representation. Interface sidechains are shown in stick representation. The hypothetical models were created by superposing the $\alpha$-helical subdomains from crystal structures of $A t$ Rca (PDB: 4W5W) (Hasse et al., 2015) and NtRca (PDB: 3T15) (Stotz et al., 2011) onto NosRca. Sequence differences to Nostoc and sidechain conformers were manually adjusted. (D) Structural rearrangement of the Rubisco substrate binding pocket by NosRca from the closed state with inhibitory sugar phosphate (ISP) bound to the open state (ISP released). (E) Cooperation of the SSUL domains and the AAA+ core of NosRca in Rubisco interaction during carboxysome biogenesis. The model is based on the interactions observed in the cryo-EM reconstructions of NosRca $\triangle \mathrm{C}$ :Rubisco and NosRca:Rubisco (showing NosSSULRubisco interaction) in the absence of nucleotide. The dotted lines represent the $\sim 35$ residue flexible linker between the AAA+ core (green) and the SSUL domains (red). 


\section{METHODS}

\section{Plasmids and Proteins}

\section{$\underline{\text { Plasmids }}$}

pET11a-NosGroSEL was generated by amplifying the groES-groEL operon by PCR from genomic DNA of Nostoc sp. PCC 7120 (NosGroSEL) and cloning into the pET11a vector via Gibson assembly. The Rubisco genes from Nostoc sp. PCC 7120 (NosrbcL and NosrbcS) were also amplified from genomic DNA by PCR and inserted into the pET28b vector. The His6ubiquitin tag $\left(\mathrm{H}_{6} \mathrm{ubi}\right)$ was amplified from the pHUE plasmid (Baker et al., 2005; Catanzariti et al., 2004) and inserted as a N-terminal fusion to NosrbcLS to obtain pET28b-H6ubi-NosLS. Finally, $\mathrm{H}_{6} \mathrm{ubi}-$ NosLS was inserted C-terminally into pET11a-NosGroSEL to obtain pET11aNosGroSEL-H6ubi-NosLS. The N-terminal deletion mutant pET11a-NosGroSEL-H6ubiNosL $\Delta$ N12-S was generated by deleting the first 12 residues (MSYAQTKTQTKS) at the Nterminus of NosRbcL by PCR. The plasmid pET11a-AtCpn $60 \alpha / \beta-A t \mathrm{Cpn} 20-N t \mathrm{RbcL}$, a kind gift from the group of M.R. Hanson (Lin et al., 2019), was modified to generate pET11a$A t \mathrm{Cpn} 60 \alpha / \beta-A t \mathrm{Cpn} 20-\mathrm{H}_{6} \mathrm{ubi}-N t \mathrm{RbcL} \Delta \mathrm{N} 9$ by deletion of the first nine $\mathrm{N}$-terminal residues (PQTETKASV) of $N t$ RbcL ( $N t$ RbcL $\Delta N 9)$, and subsequently introducing the His6-ubiquitin tag ( $\mathrm{H}_{6} \mathrm{ubi}$ ) from the pHUE plasmid as a $\mathrm{N}$-terminal fusion to $N t \mathrm{RbcL} \Delta \mathrm{N} 9$ (H6ubi-NtRbcL $\Delta \mathrm{N} 9$ ). pHUE-NosRca was generated by amplification of the $r c a$ gene from genomic DNA (Nostoc sp. PCC 7120) and subsequent cloning of residues 2-414 into the pHUE vector by using the SacII and EcoRI restriction sites. To generate pHUE-NosRca $\Delta \mathrm{C}$, residues 292 and 293 in pHUE-NosRca were changed to two consecutive stop codons by site-directed mutagenesis. All mutations in pHUE-NosRca and pHUE-NosRca $\Delta \mathrm{C}$ were introduced by QuikChange mutagenesis (Agilent) resulting in the following constructs: pHUE-NosRca mutant 
(V91E/R92G/G93N/L244D/N248A/L250A); pHUE -NosRcaAC (V91E/R92G/G93N); pHUE -

NosRca $\Delta \mathrm{C}$ (P140G/Y143A/D144A); pHUE -NosRca $\Delta \mathrm{C}$ (L244D/N248A/L250A).

pHUE-NosRca-SSUL was generated by cloning residues 325-414 (SSUL domain) of

NosRca into the pHUE plasmid by PCR, and subsequent Gibson assembly (NEB).

\section{$\underline{\text { Proteins }}$}

$N t$ Rubisco from Nicotiana tabacum leaves and $N t$ Rca(R294V) recombinantly expressed in $E$. coli, were purified as previously described (Servaites, 1985) (Stotz et al., 2011).

\section{Protein Expression and Purification}

Protein concentrations were determined spectrophotometrically at $280 \mathrm{~nm}$.

\section{NosRca $\triangle C$ and NosSSUL for crystallography}

E. coli BL21, harboring arabinose-inducible pBAD33-EcGroSEL and pHUE-NosRca plasmids, was used for the expression and purification of Nos Rca $\Delta \mathrm{C}$ (residues 2-291) as a $\mathrm{H}_{6}$-ubiquitin ( $\mathrm{H}_{6} \mathrm{Ubi}$ ) fusion protein. Cells were grown in Luria-Bertani (LB) media at $37^{\circ} \mathrm{C} / 180 \mathrm{rpm}$ until $\mathrm{OD}_{600}$ 0.3-0.4. The chaperonin GroES/EL was induced by addition of $1 \%(\mathrm{w} / \mathrm{v})$ L-arabinose. Two hours after induction, the temperature was reduced to $18^{\circ} \mathrm{C}$ and expression of $\mathrm{H}_{6} \mathrm{Ubi}_{-}$ NosRca $\Delta \mathrm{C}$ was induced by addition of $0.5 \mathrm{mM}$ isopropyl $\beta$-D-1-thiogalactopyranoside (IPTG) for $20 \mathrm{~h}$. Cells were resuspended in ethanolamine (ETA) lysis buffer (50 mM ETA pH 8.0/300 $\mathrm{mM} \mathrm{NaCl} / 10 \mathrm{mM}$ imidazole/5 \% (v/v) glycerol) containing 1 mM PMSF, 10 mM 2-

mercaptoethanol (2-ME), $1 \mathrm{~g} \mathrm{~L}^{-1}$ lysozyme and $5 \mathrm{U} \mathrm{mL}^{-1}$ benzonase, and lysed by sonication (15 x $15 \mathrm{~s}$ bursts with $75 \mathrm{~s}$ intermissions on ice). The supernatant obtained after high speed centrifugation $\left(20000 \mathrm{x}\right.$ g for $30 \mathrm{~min}$ at $4{ }^{\circ} \mathrm{C}$ ) was loaded on a gravity nickel-nitrilotriacetic acid (Ni-NTA) metal affinity column (Qiagen), washed with 10 column volumes (CV) of ETA lysis buffer $\mathrm{pH} 9.2$ containing $25 \mathrm{mM}$ imidazole, and the protein eluted with ETA lysis buffer $\mathrm{pH}$ 
9.2/200 $\mathrm{mM}$ imidazole. Fractions containing the protein were pooled and the $\mathrm{H}_{6} \mathrm{Ubi}$ moiety was cleaved by Usp2 (Baker et al., 2005; Catanzariti et al., 2004) at $4{ }^{\circ} \mathrm{C}$ overnight. After dialysis against $50 \mathrm{mM}$ ETA pH 9.2/10 mM NaCl, NosRca $\Delta \mathrm{C}$ was loaded on a Mono Q HR 16/10 column (GE). The protein was eluted with a $10 \mathrm{CV}$ gradient $(0.01-0.5 \mathrm{M} \mathrm{NaCl})$, concentrated and applied to a size-exclusion chromatography column (Superdex 200 10/300 GL; GE) equilibrated in $20 \mathrm{mM}$ Tris- $\mathrm{HCl} \mathrm{pH} 8.0 / 50 \mathrm{mM} \mathrm{NaCl} / 5 \mathrm{mM} \mathrm{MgCl}_{2}$. Fractions containing the Nos Rca $\Delta \mathrm{C}$ were concentrated by ultrafiltration using Vivaspin MWCO 10000 (GE) and glycerol added to $5 \%$ final prior to aliquoting and flash freezing in liquid $\mathrm{N}_{2}$. For all other studies the purification strategy was modified as stated below.

NosSSUL (residues 325-414) was recombinantly expressed in E. coli from the pHUENosRca-SSUL plasmid as a $\mathrm{H}_{6} \mathrm{Ubi}$ fusion protein. Cells were grown in LB media at $30^{\circ} \mathrm{C} / 180$ rpm until $\mathrm{OD}_{600} 0.3-0.4$, and then NosSSUL expression induced by addition of $0.2 \mathrm{mM}$ IPTG and the cells shifted to $22^{\circ} \mathrm{C} / 120 \mathrm{rpm}$ for $18 \mathrm{~h}$. Cells were harvested and resuspended in ETA lysis buffer (50 mM ETA pH 9.2/300 mM NaCl/10 mM imidazole/5 \% (v/v) glycerol) containing $1 \mathrm{mM}$ PMSF, $10 \mathrm{mM}$ 2-mercaptoethanol (2-ME), $1 \mathrm{~g} \mathrm{~L}^{-1}$ lysozyme and $5 \mathrm{U} \mathrm{mL}^{-1}$ benzonase, and lysed by sonication ( $15 \times 15$ s bursts with 75 s intermissions on ice). Purification of NosSSUL was carried out essentially as above for $\operatorname{Nos} \mathrm{Rca} \Delta \mathrm{C}$. Subsequent to dialysis after the first Ni-NTA column, NosSSUL was applied to a second Ni-NTA resin to remove Usp2, $\mathrm{H}_{6} \mathrm{Ubi}$ and any uncleaved protein. The flow through was concentrated and applied to a size-exclusion chromatography column (HiLoad 16/60 Superdex 75; GE) equilibrated in 50 mM ETA pH 9.2/300 mM NaCl/1 mM DTT/5 \% glycerol. Fractions containing the Nos SSUL were concentrated by ultrafiltration using Vivaspin MWCO 3000 (GE), aliquoted and flash frozen in liquid $\mathrm{N}_{2}$. 


\section{NosRca, NosRca $\triangle C$ and mutants}

NosRca, NosRca $\Delta \mathrm{C}$ and mutants were expressed and purified from E. coli BL21 STAR cells harboring arabinose-inducible pBAD33-EcGroSEL and the respective pHUE plasmid. Briefly, cells were grown in 2xYT media containing $10 \mathrm{mM} \mathrm{KCl}$ (Larimer and Soper, 1993) at $37^{\circ} \mathrm{C} / 180$ rpm until $\mathrm{OD}_{600}$ 0.3-0.4. GroSEL was induced by addition of $0.4 \%(\mathrm{w} / \mathrm{v})$ L-arabinose. One hour after chaperonin induction, the temperature was reduced to $18^{\circ} \mathrm{C}$. When the cells had equilibrated to $18{ }^{\circ} \mathrm{C}(\sim 1 \mathrm{~h})$, induction of protein was started by addition of $0.2 \mathrm{mM}$ isopropyl $\beta$ D-1-thiogalactopyranoside (IPTG) and allowed to proceed for $18 \mathrm{~h} / 120 \mathrm{rpm}$. Cells were harvested and incubated in buffer A (50 mM MMT pH 8.0/300 mM KCl/10 mM MgCl $2 / 5 \%$ glycerol) containing $1 \mathrm{~g} \mathrm{~L}^{-1}$ lysozyme/2.5 $\mathrm{U} \mathrm{mL}^{-1} / \mathrm{SmDNAse} /$ complete protease inhibitor cocktail (Roche) for $1 \mathrm{~h}$ prior to lysis using EmulsiFlex C5 (Avestin, Inc). Note MMT is a composite buffer consisting of DL-malic acid, MES monohydrate and Tris base in the molar ratio $1: 2: 2$. After high speed centrifugation $\left(40000 \mathrm{x} \mathrm{g} / 40 \mathrm{~min} / 4^{\circ} \mathrm{C}\right)$ the supernatant was loaded on to a gravity TALON metal affinity column (Takara), equilibrated and washed with $10 \mathrm{CV}$ buffer A/20 mM imidazole. The bound protein was eluted with buffer A pH 8.4/200 mM imidazole, and diluted 3-fold in buffer A containing $5 \mathrm{mM} 3-[(3-$ cholamidopropyl)dimethylammonio]-1-propanesulfonate (CHAPS)/10 \% glycerol/5 mM 2-ME to a final protein concentration $<0.5 \mathrm{~g} \mathrm{~L}^{-1}$. The $\mathrm{H}_{6} \mathrm{Ubi}$ moiety was cleaved by Usp2 overnight at $10{ }^{\circ} \mathrm{C}$. The cleaved protein was buffer exchanged on a HiPrep 26/10 desalting column (GE) to 50 mM MMT pH 8.4/10 mM KCl/5 \% glycerol, and subsequently loaded on a MonoQ column (GE). The protein was eluted with a $10 \mathrm{CV}$ gradient $(0.01-0.5 \mathrm{M} \mathrm{KCl})$, concentrated to $\sim 5 \mathrm{~mL}$ and applied onto a size-exclusion chromatography column (HiLoad 16/60 Superdex 200; GE) equilibrated in buffer B (50 mM MMT pH 8.4/100 mM KCl/10 mM MgCl$/ 2 / 5 \%$ glycerol). The 
protein containing fractions were concentrated by ultrafiltration using Vivaspin MWCO 3000 (GE), aliquoted and flash frozen in liquid $\mathrm{N}_{2}$. To generate reduced NosRca, $5 \mathrm{mM}$ DTT was added to all buffers after Usp2 cleavage. The oxidized NosRca purified in the absence of DTT was allowed to further air oxidize on ice for $8 \mathrm{~h}$ after the final column prior to concentrating, aliquoting and flash freezing.

\section{NosRubisco and mutants}

Nos Rubisco was expressed in E. coli BL21 STAR cells harboring the IPTG-inducible Nostoc chaperonin on pET11a-NosGroSEL-H6ubi-NosLS plasmid, in which the cleavable $\mathrm{H}_{6} \mathrm{Ubi}_{6}$ motif is attached at the N-terminus of NosRbcL. Cells were grown in $2 \times \mathrm{YT} / 10 \mathrm{mM} \mathrm{KCl}$ media (Larimer and Soper, 1993) at $37{ }^{\circ} \mathrm{C} / 180 \mathrm{rpm}$ until $\mathrm{OD}_{600} 0.6-0.8$. NosGroES/EL and $\mathrm{H}_{6}$ ubiNosRbcLS were induced by addition of $0.5 \mathrm{mM}$ IPTG and cells shifted to $22^{\circ} \mathrm{C}$ at $120 \mathrm{rpm}$ for 22 h. Cells were lysed, cell debris removed and the supernatant loaded onto a TALON resin column as described above. After $10 \mathrm{CV}$ washes in buffer $\mathrm{A} / 20 \mathrm{mM}$ imidazole the $\mathrm{H}_{6}$ Ubi-tagged Rubisco complex was eluted with buffer A pH 8.4/200 mM imidazole. Usp2-mediated digestion was performed overnight in presence of $5 \mathrm{mM} 2-\mathrm{ME}$ at $10^{\circ} \mathrm{C}$. After removal of imidazole on a HiPrep 26/10 desalting column (GE) equilibrated in buffer A, the buffer-exchanged protein eluate was applied to a TALON resin column for removal of Usp2, the cleaved $\mathrm{H}_{6} \mathrm{Ubi}$ moiety and any uncleaved protein. The flow through was concentrated to $\sim 5 \mathrm{~mL}$ and applied onto a size-exclusion chromatography column as above, equilibrated in buffer B containing $30 \mathrm{mM}$ $\mathrm{NaHCO}_{3}$. The NosRubisco containing fractions were concentrated by ultrafiltration using Vivaspin MWCO 30000 (GE), aliquoted and flash frozen in liquid $\mathrm{N}_{2}$.

\section{$\underline{\text { NtRubisco } \triangle N}$}

$N t$ Rubisco $\Delta \mathrm{N}$, with 9 residues deleted at the $\mathrm{N}$-terminus of the RbcL subunit, was expressed in E. coli BL21 STAR cells harboring the IPTG-inducible plasmids pET11a-AtCpn60 $\alpha \beta-A t C p n 20$ - 
$\mathrm{H}_{6} \mathrm{ubi}-\Delta \mathrm{N} 9 N t \mathrm{RbcL}$ and $\mathrm{pCDF}-N t \mathrm{XSR}_{1} \mathrm{R}_{2} \mathrm{~B}_{2}$ (kind gift from MR Hanson). Cells were grown in 0.1 L ZYP-5052 auto-induction media (Lin et al., 2019; Studier, 2005) at $37^{\circ} \mathrm{C} / 160 \mathrm{rpm}$ for $6 \mathrm{~h}$. IPTG induction was performed for at $23{ }^{\circ} \mathrm{C} / 120 \mathrm{rpm}$ for $22 \mathrm{~h}$. Harvested cells were re-suspended and incubated in buffer A containing $1 \mathrm{~g} \mathrm{~L}^{-1}$ lysozyme, $2.5 \mathrm{U} \mathrm{mL}^{-1}$ SmDNAse, complete protease inhibitor cocktail (Roche) for $1 \mathrm{~h}$ prior to lysis by sonication. After high speed centrifugation (40 $000 \mathrm{x} \mathrm{g} / 40 \mathrm{~min} / 4^{\circ} \mathrm{C}$ ) the supernatant was loaded on a HiTrap (GE) TALON Crude (Takara) metal affinity column, washed with $10 \mathrm{CV}$ of buffer A prior to elution by a $10 \mathrm{CV}$ linear imidazole gradient 0 to $200 \mathrm{mM}$ for separation of $\mathrm{H}_{6}$ Ubi-tagged Rubisco from $\mathrm{AtCpn} 60 \alpha \beta$. Fractions containing only $\mathrm{H}_{6}$ Ubi-tagged $\Delta \mathrm{N} 9 \mathrm{~N} t$ Rubisco were selected by immunoblotting against $N t \mathrm{RbcL}$ and $A t \mathrm{Cpn} 60 \alpha / \beta$. The pooled fractions were subjected to digestion by Usp2 (8 $\mathrm{h} / 10^{\circ} \mathrm{C}$ ) in the presence of $5 \mathrm{mM} 2-\mathrm{ME}$. The reaction was then buffer exchanged to $50 \mathrm{mM}$ MMT pH 8.4/100 mM KCl/5 \% glycerol on a HiPrep 26/10 desalting column (GE), and applied to a gravity TALON (Takara) metal affinity column for removal of Usp2, the cleaved $\mathrm{H}_{6} \mathrm{Ubi}$ moiety and any uncleaved protein. The flow through was concentrated by ultrafiltration using Vivaspin MWCO 30000 (GE), aliquoted and flash frozen in liquid $\mathrm{N}_{2}$.

\section{CABP Synthesis}

2-Carboxyarabinitol-1,5-diposphate (CABP) was synthesized according to (Pierce et al., 1980).

In brief, $100 \mu \mathrm{mol}$ RuBP was incubated with a 2-fold molar excess of $\mathrm{KCN}$ in $5 \mathrm{~mL} 0.1 \mathrm{M}$ Trisacetate $\mathrm{pH} 8.3$ for $48 \mathrm{~h}$ at $25{ }^{\circ} \mathrm{C}$. The racemic mixture of 2-carboxyribitol-1,5-diphosphate (CRBP) and CABP was treated with the cation exchange resin AG50W-X8 $\left(\mathrm{H}^{+}\right)$, filtered and freeze dried. To separate the enantiomers, the lactonized products were dissolved in $3 \mathrm{mM} \mathrm{HCl}$

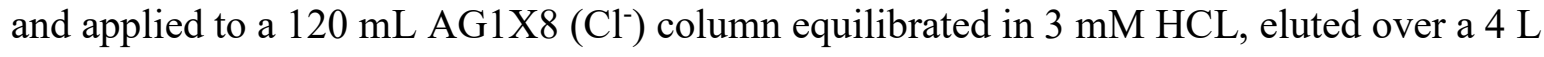


gradient from $0-0.4 \mathrm{M} \mathrm{LiCl}$, and then the $50 \mathrm{~mL}$ fractions were assayed for total phosphate (Chifflet et al., 1988). CABP containing fractions were pooled and reduced to a volume of 50 $\mathrm{mL}$ in a rotary evaporator at $30^{\circ} \mathrm{C}$. Addition of 3 -fold molar excess of barium acetate precipitated the CABP as barium salt $\left(1 \mathrm{~h}\right.$ at $\left.-20^{\circ} \mathrm{C}\right)$. The precipitate was collected by centrifugation $\left(5000 \mathrm{x} \mathrm{g}\right.$ for $20 \mathrm{~min}$ at $4{ }^{\circ} \mathrm{C}$ ) and redissolved by addition of acid-washed AG50W-X8 resin. After filtration, the purified CABP was freeze-dried and redissolved in 50 $\mathrm{mM}$ Bicine- $\mathrm{NaOH}$ pH 9.3. Complete saponification was ensured by incubation on ice for $24 \mathrm{~h}$, before aliquoting and flash freezing in liquid $\mathrm{N}_{2}$.

\section{Enzymatic Assays}

All assays were performed at $25^{\circ} \mathrm{C}$

\section{$\underline{\text { Rubisco reactivation }}$}

$\mathrm{CO}_{2}$ fixation by Rubisco and Rubisco activase activity were measured as previously described (Barta et al., 2011; Esau et al., 1996) in buffer $50 \mathrm{mM} \mathrm{MMT} \mathrm{pH} \mathrm{8.4/50} \mathrm{mM} \mathrm{KCl/30} \mathrm{mM}$ $\mathrm{NaH}^{14 / 12} \mathrm{CO}_{3}\left(14 \mathrm{~Bq} \mathrm{nmol}^{-1}\right) / 10 \mathrm{mM} \mathrm{MgCl} 2 / 3 \mathrm{mM}$ phosphocreatine/50 $\mathrm{U} \mathrm{mL}^{-1}$ phosphocreatine kinase containing RuBP and ATP as indicated in the figure legends. The carbamylated Rubisco (ECM) was obtained by preincubating Rubisco in $100 \mathrm{mM}$ MMT pH 8.4/60 mM NaHCO $3 / 20$ $\mathrm{mM} \mathrm{MgCl} 2$ for $10 \mathrm{~min}$. The uncarbamylated Rubisco (E) was obtained by buffer exchange of the purified Rubisco into $100 \mathrm{mM}$ MMT pH 8.4/50 mM KCl/4 mM EDTA. The inhibited NosRubisco (E.XuBP, E.RuBP and ECM.CABP) and inhibited NtRubisco (E.RuBP) were formed by addition of the respective inhibitory sugars and incubation for 30-60 min. Time course experiments were initiated by addition of ECM, ECM.CABP, E, E.RuBP or E.XuBP $(0.25 \mu \mathrm{M}$ active sites $)$ to the reaction assay including RuBP $(0.4 \mathrm{mM}$ unless otherwise indicated $)$, Rca and $\operatorname{ATP}(3 \mathrm{mM})$ as indicated. 
Dose dependent reactivation of NosRubisco (ECM.CABP) was measured in presence of ATP $(3 \mathrm{mM})$ and at the indicated $\operatorname{Nos} \mathrm{Rca} \Delta \mathrm{C}$ concentrations $(0.125,0.25,0.5,1.25 \mu \mathrm{M}$ hexamer). The single time-point for the amount of $\mathrm{CO}_{2}$ fixed was analyzed at 8 min after initiation of the reaction and conducted in the presence of $1 \mathrm{mM}$ RuBP to ensure steady state kinetics for the ECM control.

To estimate specific activity and account for background counts, corresponding samples were measured in 5 or 6 replicates per assay.

\section{$\underline{\text { ATPase assay }}$}

ATP hydrolysis was enzymatically coupled to NADH oxidation and measured spectrophotometrically at $25^{\circ} \mathrm{C}$ (Barta et al., 2011; Mueller-Cajar et al., 2011). The assay was started by addition of NosRca, NosRca $\Delta$ C, or mutants (NosRca V91E/R92G/G93N/

\section{L244D/N248A/L250A, NosRcaAC P140G/Y143A/D144A, NosRcaAC V91E/R92G/G93N,} $\operatorname{Nos} \mathrm{Rca} \Delta \mathrm{C}$ L244D/N248A/L250A) at $0.5 \mu \mathrm{M}$ (hexamer) in assay buffer (100 mM MMT pH 8.4/20 mM KCl/10 mM MgCl $2 / 5 \mathrm{mM}$ DTT/2 mM phosphoenolpyruvate/0.3 mM NADH/2 mM $\mathrm{ATP} / \sim 30 \mathrm{U} \mathrm{mL}^{-1}$ pyruvate kinase $/ 45 \mathrm{U} \mathrm{mL}^{-1}$ lactic dehydrogenase $) . \mathrm{ECM}$ and ECM.CABP $(0.25$ $\mu \mathrm{M}$ hexadecamer) were prepared as described above and added as indicated. To suppress turbidity due to condensate formation of activated or inhibited Rubisco (ECM or ECM.CABP, respectively) in the presence of NosRca, the salt concentration was increased to $200 \mathrm{mM} \mathrm{KCl}$ in the assay buffer.

\section{Turbidity Assay}

Measurements were performed at $25^{\circ} \mathrm{C}$ in buffer $\mathrm{C}(50 \mathrm{mM} \mathrm{MMT} \mathrm{pH} \mathrm{8.4/50} \mathrm{mM} \mathrm{KCl/10} \mathrm{mM}$ $\mathrm{MgCl}_{2}$ ) for oxidized NosRca and in the presence of additional $5 \mathrm{mM}$ DTT for reduced NosRca 
/NosRca $\Delta \mathrm{C} /$ Nos Rca-IF mut. Reactions $(100 \mu \mathrm{L})$ containing Rubisco $(0.25 \mu \mathrm{M})$ and different concentrations of NosRca or $\operatorname{Nos} \mathrm{Rca} \Delta \mathrm{C}(0.5 \mu \mathrm{M})$ or $N o s \mathrm{Rca}_{-} \mathrm{IF}_{\text {mut }}(0.5 \mu \mathrm{M})$ in the absence or presence of $2 \mathrm{mM}$ ATP $\gamma \mathrm{S}$ as indicated in the figure legends were mixed rapidly by vortexing, and absorbance at $340 \mathrm{~nm}$ was monitored as a function of time on a Jasco V-560 spectrophotometer.

\section{Liquid-liquid Phase Separation}

For analysis of LLPS, Rubisco holoenzyme was labeled at the $\mathrm{N}$ terminus with the fluorophore Alexa Fluor 532 NHS ester (ThermoFisher) according to manufacturer's instructions ( 2 dye molecules bound per Rubisco holoenzyme). Reduced NosRca, NosRca $\Delta \mathrm{C}$ and Nos Rca-IF mut was labelled at the $\mathrm{N}$ terminus with the fluorophore Alexa Fluor $405 \mathrm{NHS}$ ester (ThermoFisher) ( 2.2, 1.7 and 3 dye molecules bound per Rca hexamer, respectively). Labeled protein was mixed with unlabeled protein, at a ratio of $1: 10$. Reactions $(20 \mu \mathrm{L})$ in buffer $\mathrm{C}$ containing $5 \mathrm{mM}$ DTT with reduced NosRca, NosRca $\Delta \mathrm{C}$ or $N o s$ Rca-IF mut $(0.5 \mu \mathrm{M})$ and Rubisco $(0.25 \mu \mathrm{M})$ were incubated for $5 \mathrm{~min}$ at $25^{\circ} \mathrm{C}$ and then transferred to an uncoated chambered coverslip ( $\mu$-Slide angiogenesis; Ibidi) for another 5 min before analysis. Images were illuminated with a Lumencor SPECTRA X Light Engine at $398 \mathrm{~nm}$ and $558 \mathrm{~nm}$ for fluorescence imaging. Images were recorded by focusing on the bottom of the plate using a Leica Thunder Widefield 2 microscope with Leica DFC9000 GTC camera and a HC PL APO 63x/1.47 oil objective.

\section{Size-exclusion Chromatography Coupled to Multi-angle Static Light Scattering (SEC- MALS)}

Purified proteins at $2 \mathrm{mg} \mathrm{mL}^{-1}$ was analyzed using static and dynamic light scattering by autoinjection of the sample onto a SEC column (5 $\mu \mathrm{m}, 4.6 \times 300 \mathrm{~mm}$ column, Wyatt Technology, 
product \# WTC-030N5) at a flow rate of $0.2 \mathrm{~mL} \mathrm{~min}^{-1}$ in buffer $50 \mathrm{mM} \mathrm{MMT} \mathrm{pH} \mathrm{8.4/100} \mathrm{mM}$ $\mathrm{KCl} / 10 \mathrm{mM} \mathrm{MgCl} 2$ at $25^{\circ} \mathrm{C}$ in the presence or absence of nucleotide $(1 \mathrm{mM})$. The column was in line with the following detectors: a variable UV absorbance detector set at $280 \mathrm{~nm}$ (Agilent 1100 series), the DAWN EOS MALS detector (Wyatt Technology, $690 \mathrm{~nm}$ laser) and the Optilab $\mathrm{rEX}^{\mathrm{TM}}$ refractive index detector (Wyatt Technology, $690 \mathrm{~nm}$ laser) (Wyatt, 1993). Molecular masses were calculated using the ASTRA software (Wyatt Technology) with the dn/dc value set to $0.185 \mathrm{~mL} \mathrm{~g}^{-1}$. Bovine serum albumin (Thermo) was used as the calibration standard.

\section{Electron Microscopy and Reconstruction}

\section{$\underline{\text { Cryo-EM }}$}

All cryo-grids were prepared with a Vitrobot Mark 4 (FEI). A volume of $3 \mu \mathrm{L}$ of the sample was applied to a glow-discharged grid (Quantifoil R2/1 300 mesh) at $25{ }^{\circ} \mathrm{C}$ and $90 \%$ humidity, then semi-automatically blotted and plunge-frozen into liquid ethane.

To capture the interaction between $\operatorname{Nos} \mathrm{Rca} \Delta \mathrm{C}$ and CABP-inhibited Rubisco (NosRca $\Delta$ C:Rubisco), NosRubisco $(1 \mu \mathrm{M})$ was first carbamylated by incubation in buffer C containing $10 \mathrm{mM} \mathrm{NaHCO}_{3}$ for $10 \mathrm{~min}$ at $25^{\circ} \mathrm{C}$, then inhibited with $8 \mu \mathrm{M} \mathrm{CABP}$ at $25^{\circ} \mathrm{C}$ for 1 h. The NosRca $\Delta$ C:Rubisco (NosRca $\Delta$ C:ECM.CABP) complex was formed following a published nucleotide-substitution strategy (Dong et al., 2019). Specifically, ECM.CABP (0.5 $\mu$ M) was incubated with $\operatorname{Nos} \mathrm{Rca} \Delta \mathrm{C}(10 \mu \mathrm{M})$ at $25^{\circ} \mathrm{C}$ in the presence of ATP $(2 \mathrm{mM})$ for $10 \mathrm{~s}$, followed by the addition ATP $\gamma \mathrm{S}(2 \mathrm{mM})$, and incubated at $25^{\circ} \mathrm{C}$ for another $10 \mathrm{~min}$ before preparing the cryo-grids as stated above. The cryo-grids were initially screened on a Talos Arctica (FEI) transmission electron microscope (TEM). Selected grids were transferred to a Titan Krios 300 kV TEM (FEI) equipped with GIF Quantum Energy Filters (Gatan), and a K3 direct detector 
(Gatan). 9,042 movies were automatically collected by SerialEM (Mastronarde, 2005) using a pixel size of $0.8512 \AA$. The total exposure time of $2.8 \mathrm{~s}$ was divided into 31 frames with an accumulated dose of 60 electrons per $\AA^{2}$ and a defocus range of $-0.7 \mu \mathrm{m}$ to $-2.5 \mu \mathrm{m}$.

For Preparing the NosRca:Rubisco complex, NosRubisco $(1.25 \mu \mathrm{M})$ was mixed with Nos Rca $(15 \mu \mathrm{M})$ in buffer C containing $5 \mathrm{mM}$ DTT and cryo-grids prepared as above. The cryogrids were screened on a Glacios transmission electron microscope (Thermo Scientific), equipped with K2 summit direct electron detector (Gatan), operated at $200 \mathrm{keV}$. Selected grid on stage was used for data collection directly with K2 summit. Exposure times of $12 \mathrm{~s}$ were divided into 40 frames with an accumulated dose of 47 electrons per $\AA^{2} .1,570$ movies were automatically collected by SerialEM (Mastronarde, 2005) with a pixel size of $1.885 \AA$ and a defocus range of $-1 \mu \mathrm{m}$ to $-4.5 \mu \mathrm{m}$.

\section{Image processing}

For the NosRca $\Delta \mathrm{C}$ :Rubisco $(\operatorname{Nos} \mathrm{Rca} \Delta \mathrm{C}$ :ECM·CABP) dataset, on-the-fly processing during data collection was performed with MotionCorr2 and CTFFIND-4.1, as implemented in the Focus software (Biyani et al., 2017). Only micrographs with good particle quality, with an estimated maximum resolution below $5 \AA$, were kept for further data processing with RELION 3.0. A total of 519,151 particles were auto-picked by Gautomatch (http://www.mrc-

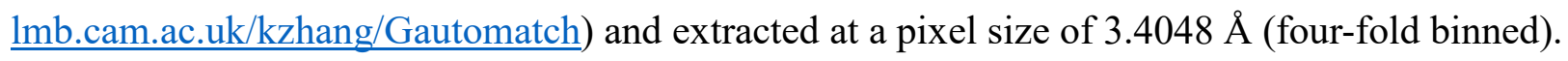
The first round of 2D-classification was used to exclude ice contaminations and classes with no structural features. The selected particles were next refined according to a Rubisco reference converted from the dataset of PDB 1RBL (Newman et al., 1993), re-centered, and then subjected to a second round of 2D-classification. Free $\operatorname{Nos} \mathrm{Rca} \Delta \mathrm{C}$ classes were excluded, which resulted in the selection of 106,831 particles (Figure S3). 3D-classification with the Rubisco reference 
showed only one class of the NosRca $\Delta \mathrm{C}$ hexamer in complex with Rubisco, containing 30,607 particles. These particles were re-extracted at full resolution $(0.8512 \AA$ pixel size $)$ and subjected to "polishing" with RELION to generate "shiny" particles. To deal with multiple Nos Rca $\triangle \mathrm{C}$ hexamers bound per Rubisco, we followed a previously published symmetry-expansion procedure (Wang et al., 2019). In detail, particles were first aligned with $D 4$ symmetry. Then $D 4$ symmetry was released and yielded 8-fold particles by the symmetry-expanding command, relion_particle_symmetry_expand. A focused 3D-classification in NosRca $\Delta \mathrm{C}$ with symmetryreleased particles revealed one class with $\operatorname{Nos} \mathrm{Rca} \Delta \mathrm{C}$ hexamer occupancy, comprising of 21,149 particles (Figure S3). These particles generated the final map of the NosRca $\Delta \mathrm{C}: \mathrm{ECM} \cdot \mathrm{CABP}$ complex at $2.86 \AA$ resolution, determined by gold-standard Fourier shell correlation (FSC) with a cutoff at 0.143 . Particle subtraction of the Rubisco signal improved the alignment accuracy of the NosRca $\Delta \mathrm{C}$ hexamer, and generated a local NosRca $\Delta \mathrm{C}$ map at $3.29 \AA$ resolution.

The raw movies of the NosRca:Rubisco dataset were first processed with MotionCorr2 (Zheng et al., 2017) with dose-weighting. CTFFIND-4.1(Rohou and Grigorieff, 2015) estimated the CTF parameters for each micrograph. 298,336 Particles were picked by Gautomatch (http://www.mrc-lmb.cam.ac.uk/kzhang/Gautomatch). Two rounds of 2D-classification excluded ice contaminations and classes with no structural features, and resulted in 45,859 clean particles (Figure S7). These particles were used to generate a reference for 3D-classification in RELION 3.0. (Scheres, 2012) with the 3D initial model module. 3D-classification revealed one class with detailed Rubisco features (27,527 particles). To deal with multiple SSUL domains bound per Rubisco, we followed the same symmetry-expansion procedure previously reported (Wang et al., 2019). Particles were first aligned with $D 4$ symmetry. Each asymmetric unit $\mathrm{L}_{2} \mathrm{~S}_{2} \mathrm{SSUL}$ was processed as an individual particle, which is achieved by the symmetry-expanding command, 
relion_particle_symmetry_expand, and particle subtraction. A focused classification with a SSUL mask resulted in one class of particles with detailed SSUL feature. 32,128 particles from this class were selected and subjected to final local refinement. Post-processing improved the map resolution to $8.2 \AA$.

Model building

Nos Rca $\Delta \mathrm{C}: \mathrm{ECM} \cdot \mathrm{CABP}-\operatorname{Nos} \mathrm{Rca} \Delta \mathrm{C}$ model building was initiated by rigid-body fitting the NosRca $\Delta \mathrm{C}$ subdomains from the crystal structure into the cryo-EM density, followed by manual editing using Coot (Emsley and Cowtan, 2004). This model was refined in reciprocal space with REFMAC5 (Murshudov et al., 2011). The ECM.CABP model was generated with SWISSMODEL (Waterhouse et al., 2018) based on the coordinates of Rubisco from Chlamydomonas reinhardtii (PDB 1UZH) (Karkehabadi et al., 2005). This model was placed into the cryo-EM density using Chimera (Pettersen et al., 2004), followed by manual editing using Coot. Residues with disordered side-chains were truncated at $\mathrm{C}-\beta$. This model was refined in reciprocal space with REFMAC5 (Murshudov et al., 2011), using non-crystallographic symmetry restraints.

NosRca:Rubisco - First, the crystal structures of thiol-reduced NosSSUL and NosRubisco were placed into the density using Chimera, followed by manual editing using Coot. The resulting model was refined in reciprocal space with REFMAC5, using jelly-body restraints. The used structure factors were calculated from a masked map.

The models and the electron density maps for NosRca $\Delta \mathrm{C}$ :Rubisco and NosRca Rubisco have been deposited to the wwPDB database under PDB/EMDB accession codes 6Z1F/EMD11028 and 6Z1F/EMD-11029, respectively.

\section{Crystallization and Data Collection}


The Nos Rca $\Delta \mathrm{C}$ construct used for crystallization includes residues $2-291$. The Nos SSUL construct used for crystallization includes residues 325-414.

$\operatorname{Nos} \mathrm{Rca} \Delta \mathrm{C}-$ Crystals were grown by the hanging-drop vapor diffusion method at $4{ }^{\circ} \mathrm{C}$. Drops containing $2 \mu \mathrm{L}$ of a 1:1 mixture of $4.9 \mathrm{mg} \mathrm{mL}^{-1}$ NosRca $\Delta \mathrm{C}$ in buffer $20 \mathrm{mM}$ Tris- $\mathrm{HCl} \mathrm{pH}$ 8.0/50 $\mathrm{mM} \mathrm{NaCl} / 5 \mathrm{mM} \mathrm{MgCl}_{2}$ and precipitant were equilibrated against $500 \mu \mathrm{L}$ precipitant. The precipitant contained 2.2 or $2.3 \mathrm{M} \mathrm{Na}$-acetate $\mathrm{pH}$ 7.0.

NosSSUL - Crystals were grown by the hanging-drop vapor diffusion method at $4{ }^{\circ} \mathrm{C}$. Drops containing $3 \mu \mathrm{L}$ of a 1:1 mixture of $5.2 \mathrm{mg} \mathrm{mL}^{-1}$ NosSSUL in buffer $50 \mathrm{mM}$ ETA pH 9.2/300 mM NaCl/1 mM DTT/5 \% glycerol and precipitant were equilibrated against $500 \mu \mathrm{L}$ precipitant. The precipitant contained $26 \%$ PEG-3350 and $50 \mathrm{mM}$ MES-NaOH pH 6.0.

For cryo-mounting, the crystals were transferred into a cryo-solution that was precipitant containing $15 \%$ glycerol in addition and subsequently cryo-cooled by dipping into liquid nitrogen.

\section{Crystallographic data collection, structure solution and refinement}

Nos Rca $\Delta \mathrm{C}$ - The diffraction data of the crystals of NosRca $\Delta \mathrm{C}$ were collected by the oscillation method at beamline X06DA at the Swiss Synchrotron Light Source (SLS) in Villigen, Switzerland. The diffraction data from $\operatorname{Nos} \mathrm{Rca} \Delta \mathrm{C}$ crystals were integrated with XDS and further processed with POINTLESS (Evans, 2006), SCALA (Evans, 1997) and CTRUNCATE (French and Wilson, 1978). The structure was solved at $3.4 \AA$ from a Gadolinium $\left(\mathrm{GdCl}_{3}\right)$ derivative by Gd-multi-wavelength anomalous diffraction (MAD) using ShelxC/D/E (Sheldrick, 2010) as implemented in the Hkl2map GUI (Pape and Schneider, 2004). The seven Gd sites were refined and phases calculated with SHARP (de la Fortelle and Bricogne, 1997). The map, calculated after density modification with RESOLVE (Terwilliger, 2000), assuming a solvent content of 
$55 \%$, revealed features of secondary structure elements. A preliminary model was auto-built with Buccaneer (Cowtan, 2006), and missing portions added manually using COOT (Emsley and Cowtan, 2004). REFMAC5 was used for initial model refinement (Murshudov et al., 2011). The final refinement was performed with phenix.refine (Adams et al., 2010) using translationlibration-screw (TLS) parametrization of B-factors. The native structure was solved by molecular replacement. The final models contain two copies of $N o s \mathrm{Rca} \Delta \mathrm{C}$ per asymmetric unit. One chain (chain B) has ADP bound. Residues 276-291 were disordered in chain A. In chain B, residues 105-115, 248-254 and 279-291 are missing. Residues facing solvent channels with disordered side-chains were modelled as alanine. The model of the native structure contains 14 ordered water molecules and exhibits reasonable stereochemistry with $96.6 \%$ of the residues in the favored regions of the Ramachandran plot according to the criteria of MolProbity (Chen et al., 2010).

NosSSUL - The native diffraction data of the crystals of NosSSUL were collected at the automated beamline ID30A-1 at the European Synchrotron Radiation Facility (ESRF) in Grenoble, France. MAD data for a presumed Pt-derivative were collected at beamline BM30A at ESRF. The diffraction data from NosSSUL crystals were integrated with XDS and further processed with POINTLESS (Evans, 2006), AIMLESS (Evans and Murshudov, 2013) and CTRUNCATE (French and Wilson, 1978) as implemented in the CCP4i graphical user interface (Potterton et al., 2003). The structure of NosSSUL was solved by MAD using the Auto-Rickshaw platform (Panjikar et al., 2005). The anomalous scatterers were a bound $\mathrm{Ni}^{2+}$ atom from protein purification and presumably ordered $\mathrm{Cl}^{-}$ions. The chemical environment of the sites was not compatible with $\mathrm{PtCl}_{4}{ }^{2-}$. The asymmetric unit contained two copies of the SSUL domain. The model was edited manually using Coot (Emsley and Cowtan, 2004). REFMAC5 was used for 
model refinement (Murshudov et al., 2011). The model contains 203 ordered water molecules and exhibits reasonable stereochemistry with $99.5 \%$ of the residues in the favored regions of the Ramachandran plot according to the criteria of MolProbity (Chen et al., 2010).

Figures were created with PyMol (http://www.pymol.org/) and ESPript (Gouet et al., 1999).

\section{Structure Analysis}

The quality of the structural models was analyzed with the program Molprobity (Chen et al., 2010). Coordinates were aligned with Lsqkab and Lsqman (Kleywegt and Jones, 1994).

Molecular interfaces were analyzed with PISA (Krissinel and Henrick, 2007) and Contact, as implemented in the CCP4i graphical user interface (Potterton et al., 2003). Figures were created with Chimera (Pettersen et al., 2004), PyMol (http://www.pymol.org/) and ESPript (Gouet et al., 1999).

\section{Data Resources}

The models and the electron density maps for NosRca $\Delta \mathrm{C}$ :Rubisco and NosRca Rubisco have been deposited to the wwPDB database under PDB/EMDB accession codes 6Z1F/EMD-11028 and 6Z1F/EMD-11029, respectively. The crystallographic models and structure factors for Nos Rca $\Delta$ C-Gd complex, NosRca $\Delta \mathrm{C}$ and NosSSUL have been deposited to the PDB database under accession codes 6Z1D, 6Z1E and 6HAS, respectively. 


\section{SUPPLEMENTAL FIGURES}
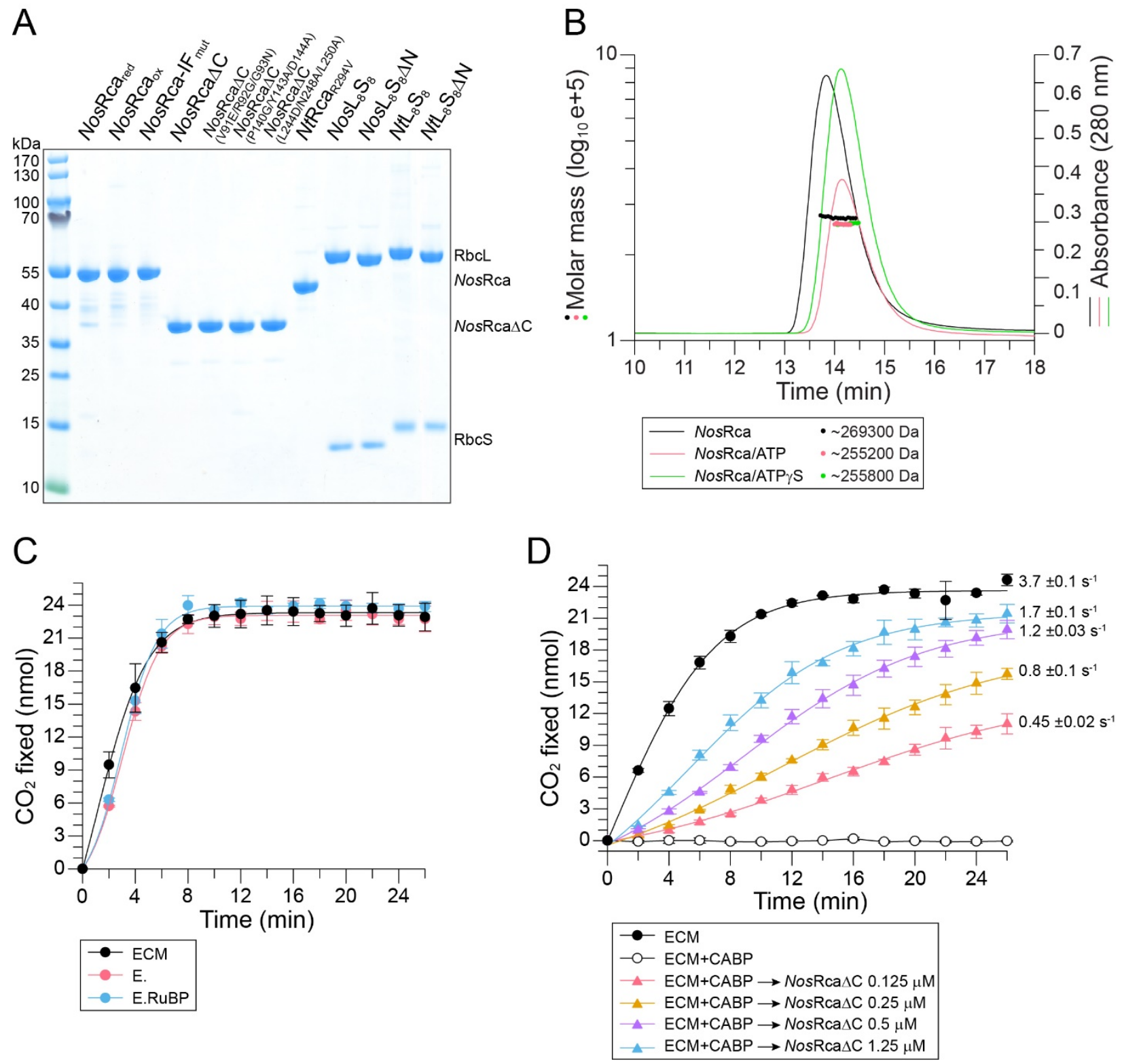

Figure S1. Rubisco Activase Function of NosRca.

(A) Purified proteins used in this study. Protein concentrations were determined spectrophotometrically. $1.5 \mu \mathrm{g}$ protein was analyzed by SDS-PAGE. (B) NosRca is a hexamer in solution. SEC-MALS analysis of NosRca in the absence or presence of nucleotide (1 mM ATP or ATP $\gamma \mathrm{S}$ ). The chromatographic absorbance traces at $280 \mathrm{~nm}$ wavelength are shown. The molecular mass determined for the protein peaks by static light scattering is indicated. (C) Noncambamylated NosRubisco is not inhibited by RuBP. $\mathrm{CO}_{2}$ fixation assays were performed with carbamylated (ECM), non-carbamylated (E) and non-carbamylated Rubisco with RuBP (E.RuBP) as in Figure 1C. Error bars represent SD of at least three independent replicates. (D) Dependence of Rubisco reactivation on NosRca $\Delta \mathrm{C}$ concentration. CABP inhibited NosRubisco 
bioRxiv preprint doi: https://doi.org/10.1101/2020.05.16.099382; this version posted May 16, 2020. The copyright holder for this preprint (which

was not certified by peer review) is the author/funder, who has granted bioRxiv a license to display the preprint in perpetuity. It is made available under aCC-BY-NC-ND 4.0 International license.

(ECM.CABP) was incubated with increasing concentrations of $N o s \mathrm{Rca} \Delta \mathrm{C}(0.125,0.25,0.5$ and $1.25 \mu \mathrm{M}$ hexamer) in the presence of $3 \mathrm{mM} \mathrm{ATP}$, and $\mathrm{CO}_{2}$ fixation measured as in Figure 1D. Approximate rates of $\mathrm{CO}_{2}$ fixation were determined from the linear parts of the curves. Error bars represent SD of three independent replicates. 
bioRxiv preprint doi: https://doi.org/10.1101/2020.05.16.099382; this version posted May 16, 2020. The copyright holder for this preprint (which was not certified by peer review) is the author/funder, who has granted bioRxiv a license to display the preprint in perpetuity. It is made available under aCC-BY-NC-ND 4.0 International license.
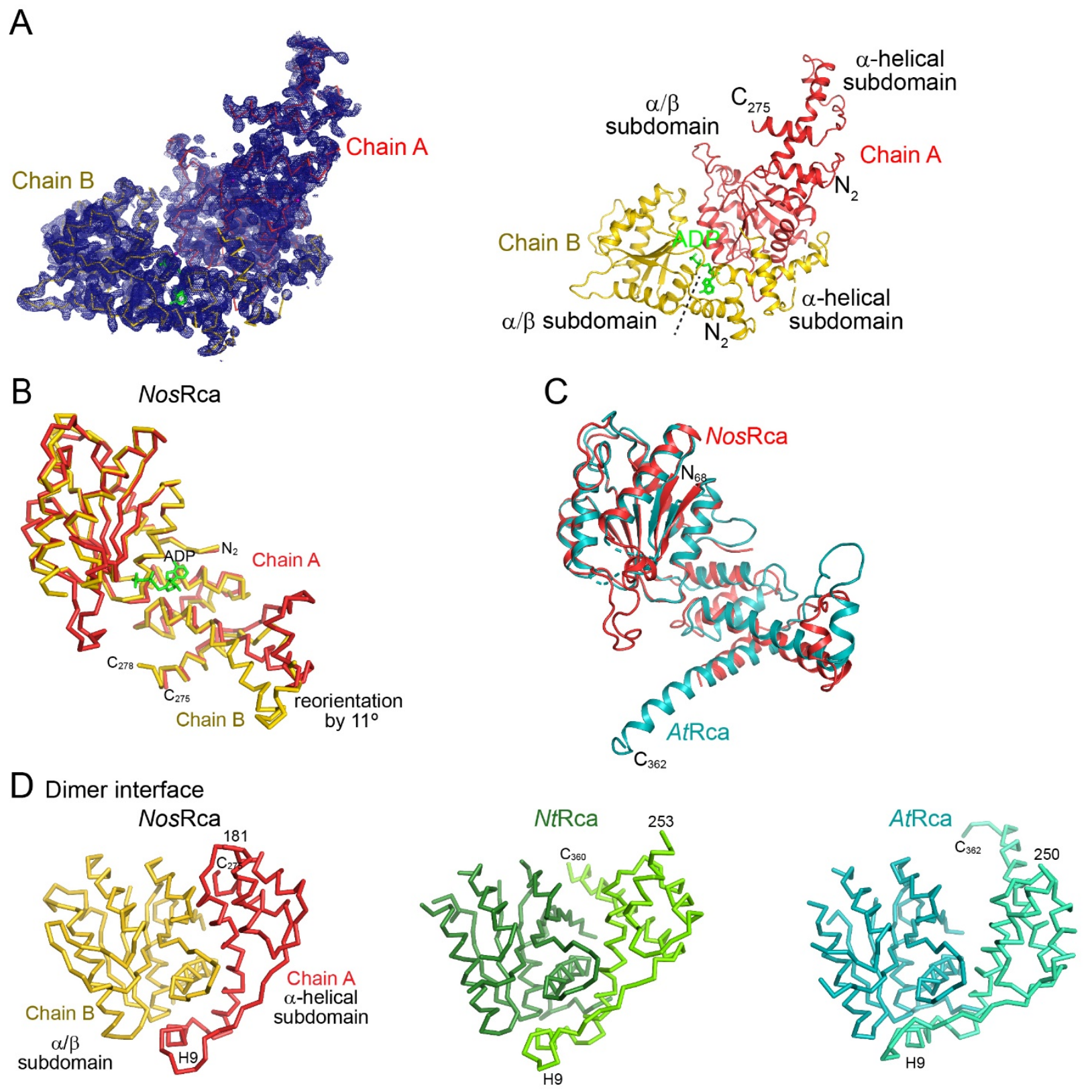

E $\alpha$-helical subdomain

NosRca

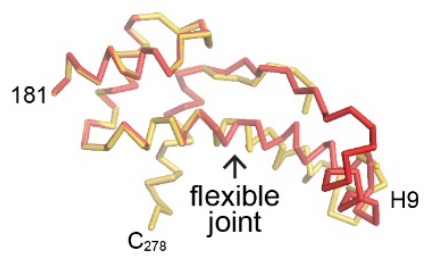

NtRca

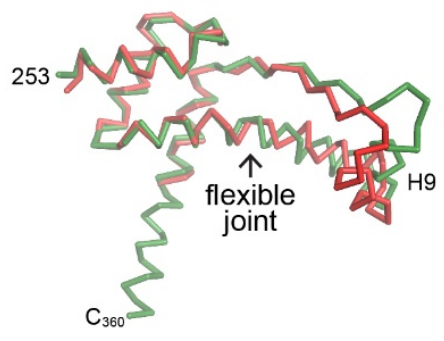

AtRca

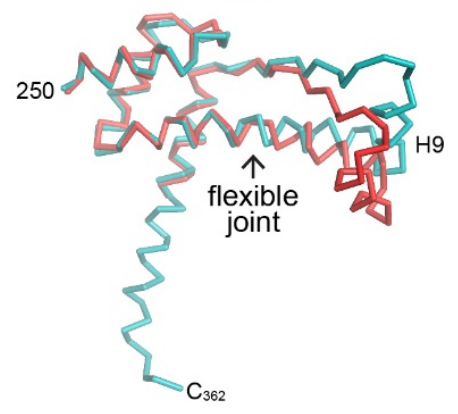


Figure S2. Crystal Structure of NosRca $\Delta$ C.

(A) Left: Unbiased experimental electron density at $2.7 \AA$ resolution for the $\mathrm{Gd}^{3+}$ complex of NosRca $\Delta \mathrm{C}$ (for data collection and refinement statistics, see Table S2). The density after GdMAD phasing and density modification contoured at $1.5 \sigma$ is shown as a blue meshwork. Chains $\mathrm{A}$ and $\mathrm{B}$ of the asymmetric unit are shown as $\mathrm{C} \alpha$ traces in red and yellow, respectively. ADP is represented as a wire-frame model in green. Right: Final model of the asymmetric unit in the NosRca $\Delta \mathrm{C}$ crystal lattice. The two crystallographically independent chains of NosRca $\Delta \mathrm{C}$ are shown as ribbons. Subdomains and chain termini are indicated. The dashed line indicates the subdomain boundary in chain B. (B) Superposition of chain A with chain B in the asymmetric unit of $\operatorname{Nos} \mathrm{Rca} \Delta \mathrm{C}$. Protein chains are represented as $\mathrm{C} \alpha$ traces. (C) Superposition of chain A (no nucleotide bound) of the asymmetric unit of NosRca $\Delta \mathrm{C}$ with $A t$ Rca (PDB: 4W5W) (Hasse et al., 2015). Chain A of NosRca $\Delta \mathrm{C}$ and $A t$ Rca are shown as ribbons in red and teal, respectively. Chain termini are indicated. (D) A conserved subunit-subunit interaction found in NosRca (left), $N t$ Rca (middle) and $A t$ Rca (right). The interaction is formed between the $\alpha / \beta$ subdomain in one subunit and the C-terminal half of helix $\alpha 8, \alpha 9$ (H9) and the connecting linker in the $\alpha$-helical subdomain of the adjacent subunit. Adjacent subunits in the $N t$ Rca crystal structure are shown in dark and bright green, and in teal and cyan for AtRca. The location of helix H9 and chain termini are indicated. Note that the orientation of helix $\alpha 9$ (H9) with respect to the four-helix bundle in the $\alpha$-helical subdomain differs between the structures; the long helix $\alpha 8$ and the long $\alpha 9-\alpha 10$ linker act as a stalk that can twist and bend like a flexible joint.

(E) Superposition of the $\alpha$-helical subdomain of chain A in NosRca with chain B (left), with $N t$ Rca (middle) and $A t$ Rca (right). The r.m.s.d. values for matching C $\alpha$ positions are $0.24 \AA$ (57 C $\alpha$ positions), $0.58 \AA$ (42 C $\alpha$ positions) and $0.55 \AA$ (47 C $\alpha$ positions), respectively. The locations of helix $\alpha 9$ (H9) and the flexible joint region are indicated. Chain termini are indicated. 
bioRxiv preprint doi: https://doi.org/10.1101/2020.05.16.099382; this version posted May 16, 2020. The copyright holder for this preprint (which was not certified by peer review) is the author/funder, who has granted bioRxiv a license to display the preprint in perpetuity. It is made available under aCC-BY-NC-ND 4.0 International license.

A

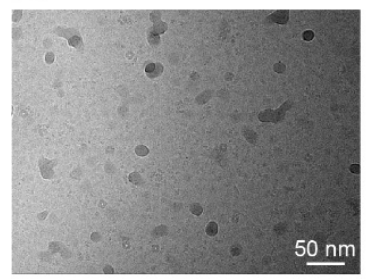

B

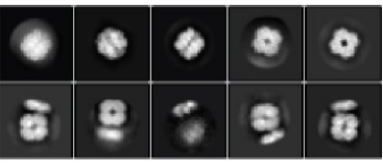

C

9,042 micrographs - Motioncorr2, CTFFIND4, Gautomatch - 2D classification with B4
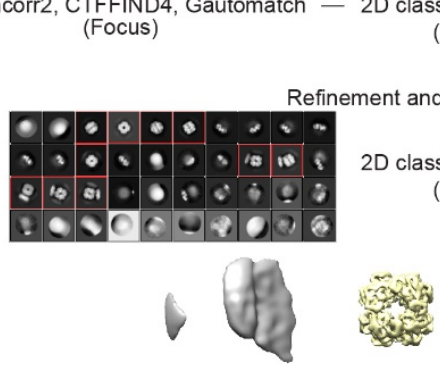

$(335,474)$

|

$(106,831)$

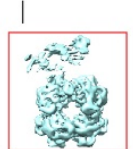

$(30,607)$

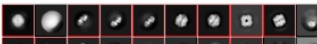

- $30.10 \mathrm{~m}$ a

Refinement with D4 - CTFRefinement and Polishing - Extraction particles with $0.8512 \AA$ ।

Symmetry expanding

Focused classification on Rca

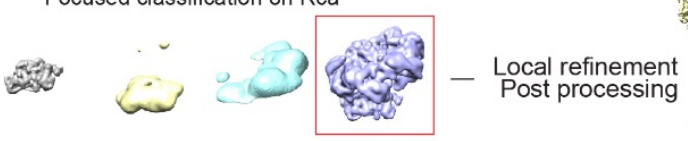

$(21,149)$
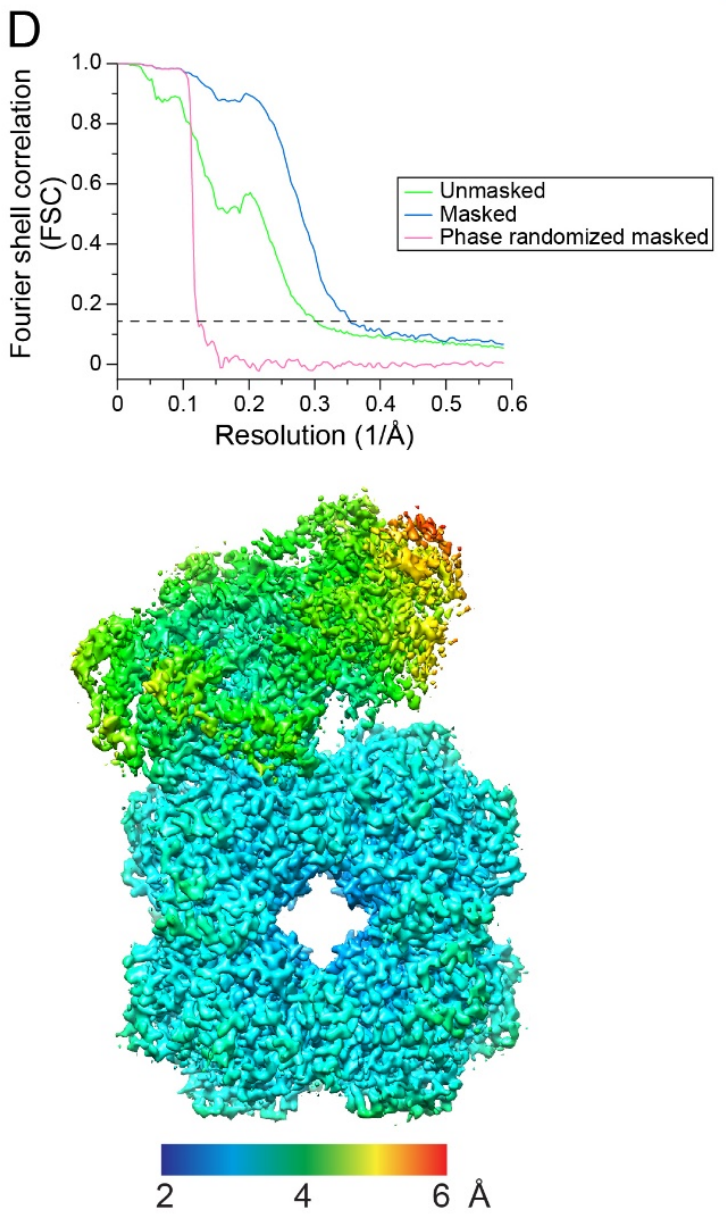

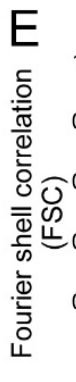

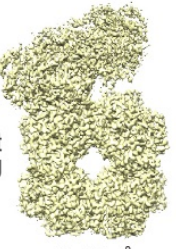

- Subtraction

$2.86 \AA$

Local refinement

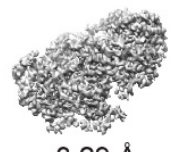

$3.29 \AA$
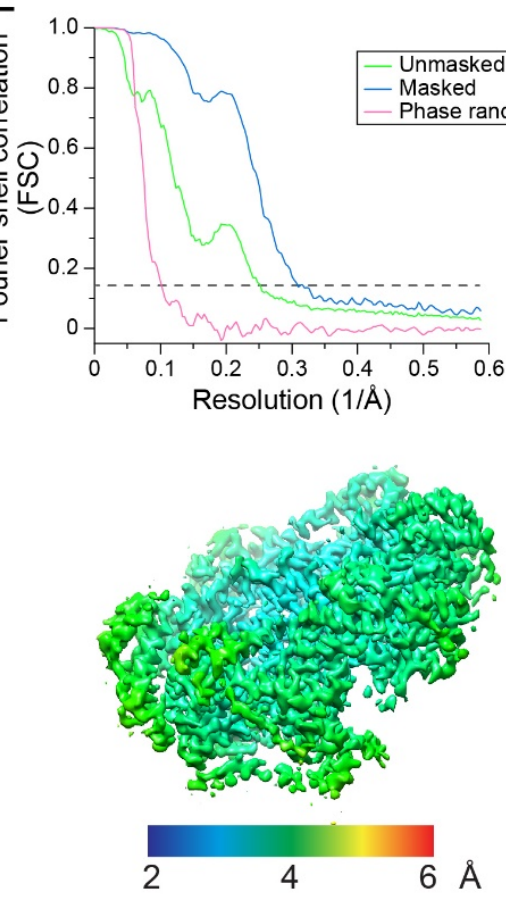
bioRxiv preprint doi: https://doi.org/10.1101/2020.05.16.099382; this version posted May 16, 2020. The copyright holder for this preprint (which

was not certified by peer review) is the author/funder, who has granted bioRxiv a license to display the preprint in perpetuity. It is made available under aCC-BY-NC-ND 4.0 International license.

Figure S3. Cryo-EM Single-particle Reconstruction of NosRca $\Delta$ C:Rubisco Complex. (A) A representative micrograph of $N o s$ Rca $\Delta$ C:Rubisco complexes. (B) $2 \mathrm{D}$ class averages of complexes in (A). (C) The single-particle data processing workflow for the NosRca $\Delta \mathrm{C}: \mathrm{Rubisco}$ complex. Particle numbers are in parentheses. B4, 4x4 pixel-binned image. See STAR Methods for details. (D and E) Gold-standard FSC curves and local resolution maps of the NosRca $\Delta$ C:Rubisco reconstruction (D) and the $N o s$ Rca $\Delta \mathrm{C}$ local map (E). The resolutions are $\sim 2.86 \AA$ and $\sim 3.29 \AA$, respectively, at the FSC cutoff of 0.143 for the masked and B-factor sharpened curves. The color gradient from blue to red indicates local resolution from 2.0 to 6.0 $\AA$. 
A
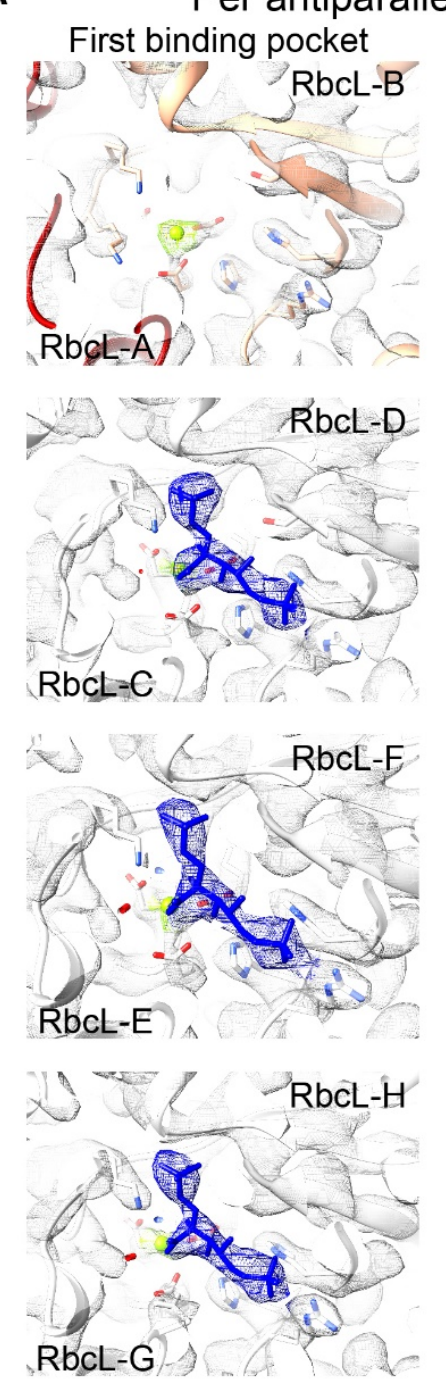

RecL dimer

Second binding pocket
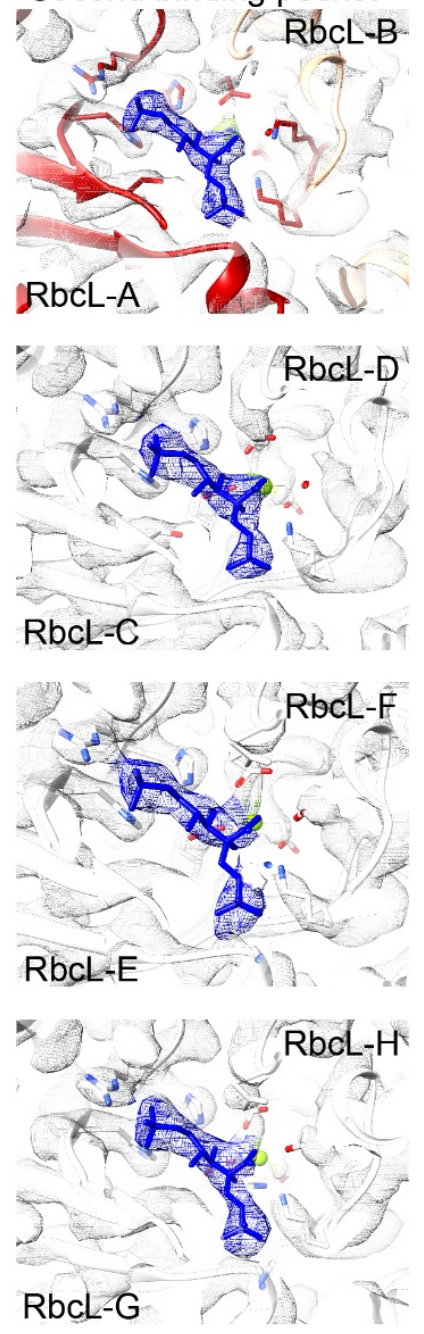

B

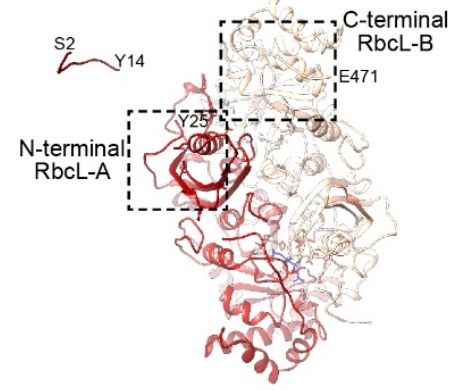

C C-terminal EM density

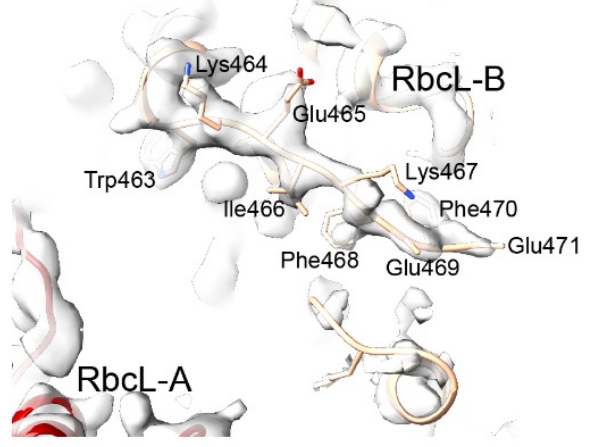

D N-terminal EM density

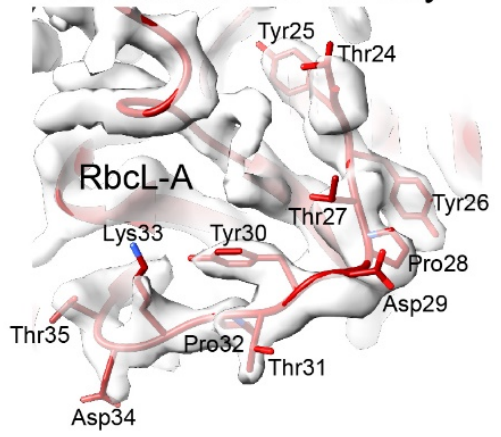

E

1 Nostoc sp. PCC 7120

Fremyella diplosiphon

Geitlerinema PCC 7407

Trichodesmium erythraeum

Cyanothece PCC 7425

Synechococcus PCC 7335

2 Ostreococcus lucimarinus

Chlamydomonas reinhardtii

Nicotiana tabacum

Arabidopsis thaliana

Spinacia oleracea

Oryza sativa

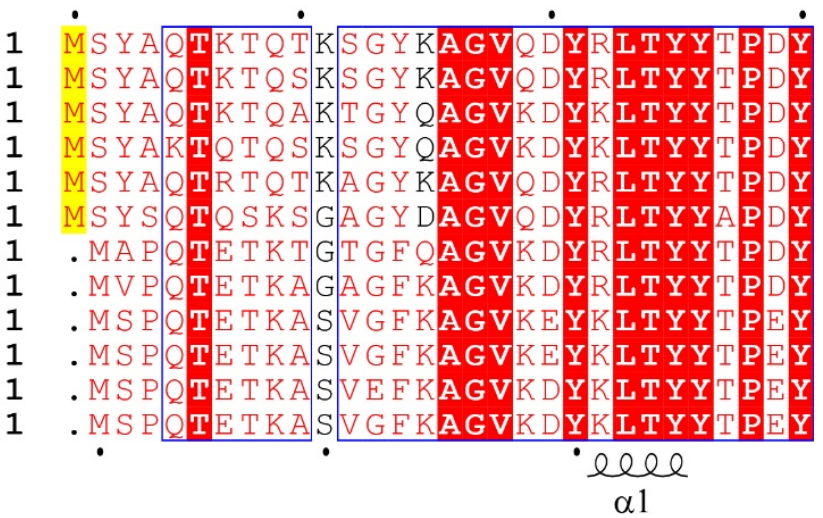


Figure S4. Key Structural Features of Inhibited NosRubisco in Complex With NosRca $\Delta$ C. (A) Close-up of the cryo-EM density of the eight substrate binding pockets of NosRubisco. Each $\mathrm{RbcL}$ anti-parallel dimer has two binding pockets. The density is represented as a transparent iso-contour surface. CABP density is shown as a meshwork in blue. Protein is represented by ribbons with ligand-interacting sidechains and CABP in stick representation. $\mathrm{Mg}^{2+}$ ions are shown as green spheres. (B) An overview of the RbcL-A/RbcL-B dimer that is engaged by NosRca $\Delta$ C. RbcL-A and RbcL-B are shown in red and peach, respectively. The N- and Cterminal residues, as well as the RbcL-A N-terminal peptide bound in the hexamer pore of NosRca $\Delta \mathrm{C}$ are indicated. (C and D) Zoomed-in views of the $\mathrm{N}$ - and $\mathrm{C}$-terminal regions, indicated by dotted boxes in (B). The EM densities of the C-terminal residues of RbcL-B (C) and the N-terminal residues of RbcL-A (D) are well-resolved in the cryo-EM density map of the NosRca $\Delta$ C:Rubisco complex. (E) Alignment of the N-terminal sequences of RbcL from selected cyanobacterial and eukaryotic form 1B Rubisco proteins. Similar residues are shown in red and identical residues in white on a red background. Blue frames indicate homologous regions. Yellow background indicates systematic differences between cyanobacterial and eukaryotic sequences. The secondary structure elements of RbcL from Oryza sativa (PDB: 1WDD) (Matsumura et al., 2012) are indicated below the sequences. The Uniprot and Genebank accession codes for the sequences are: P00879, Nostoc PCC 7120; NZ JH930358.1, Fremyella diplosiphon; K9SCF1, Geitlerinema PCC 7407; Q10WH6, Trichodesmium erythraeum; B8HQS5, Cyanothece PCC 7425; B4WP00, Synechococcus PCC 7335; Q0P3J3, Ostreococcus lucimarinus; P00877, Chlamydomonas reinhardtii; P00876, Nicotiana tabacum; O03042, Arabidopsis thaliana; P00875, Spinacia oleracea; P0C510, Oryza sativa. 
A
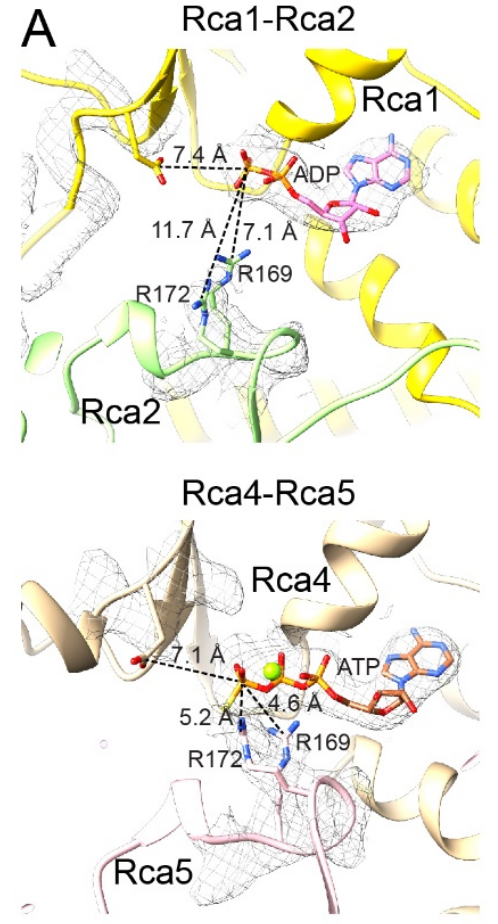

B Pore-loop 1 (PL1)

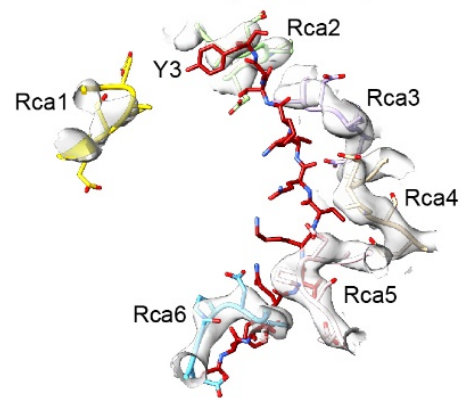

E
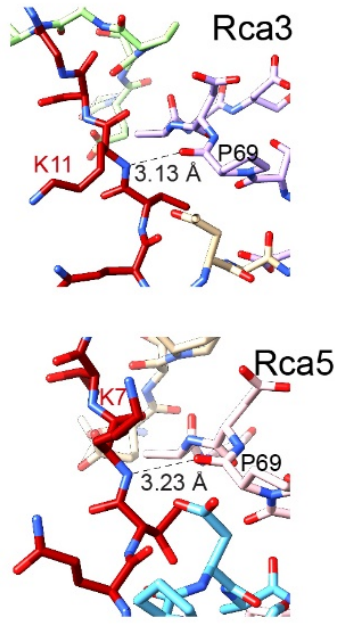

Rca2-Rca3

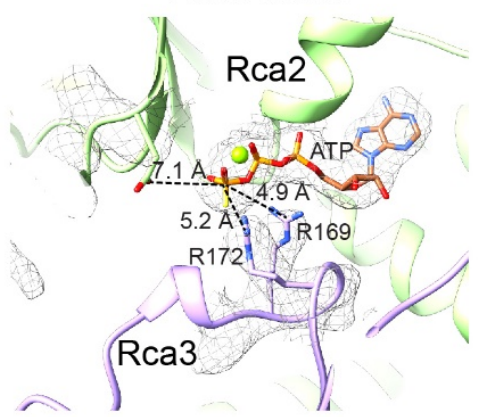

Rca5-Rca6

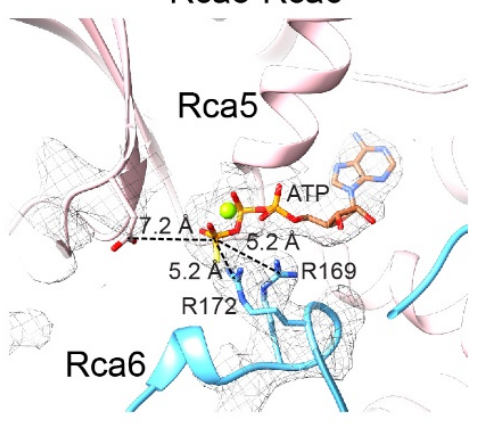

C Pore-loop 2 (PL2)

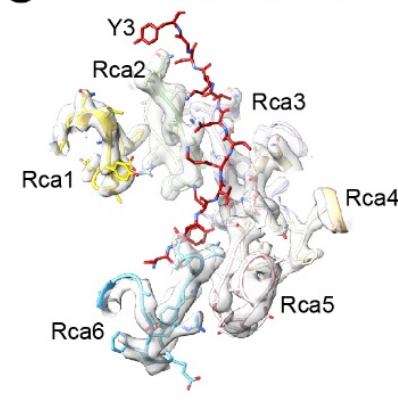

Rca3-Rca4

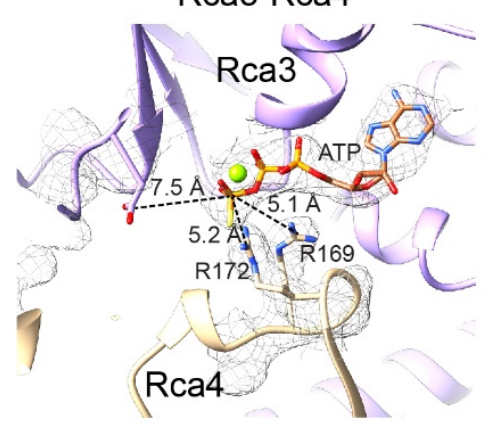

Rca6-Rca1

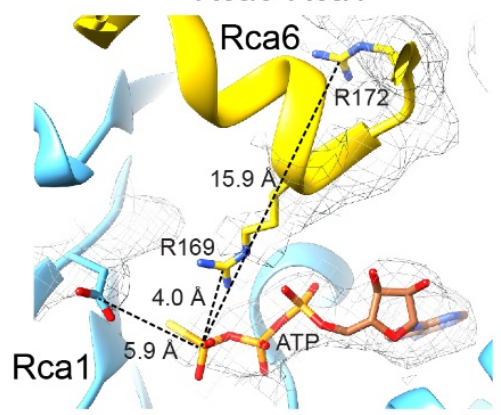

D Pore-loop 3 (PL3)

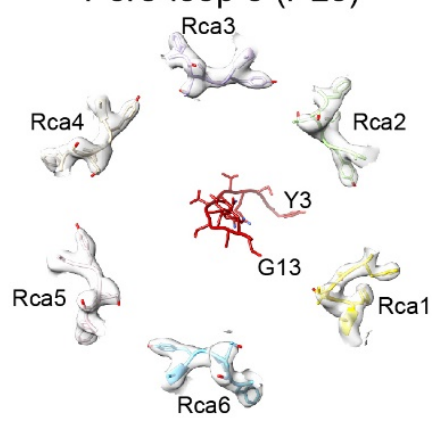

F
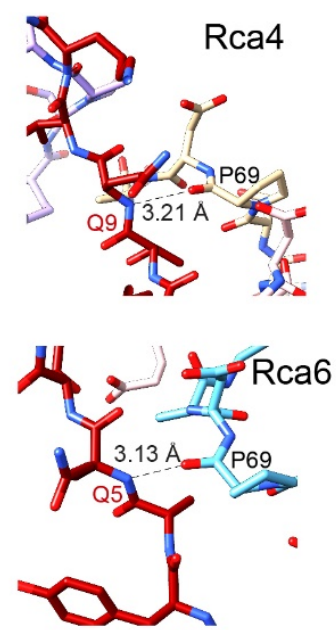

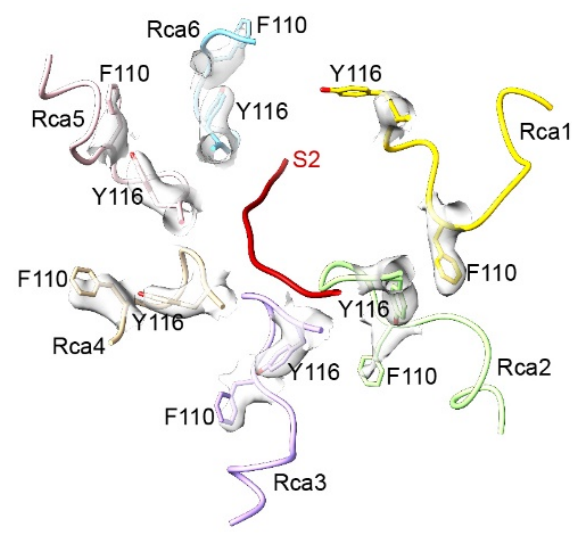


Figure S5. Key Structural Features in NosRca $\Delta$ C in the Complex with NosRubisco. (A) Zoomed-in views of the nucleotide binding pockets formed between adjacent Nos Rca subunits. Distances of the carbon atom in the guanidino group of Arg169 and Arg172, and of the carboxylate carbon of Asp102 (Walker B) to the last phosphorus atom of the nucleotide are indicated. Cryo-EM density is shown as a meshwork. The protein is shown in ribbon representation, nucleotide in stick representation and $\mathrm{Mg}^{2+}$ ion as a green sphere. (B to D) Zoomed-in views of pore-loops PL1 (B), PL2 (C) and PL3 (D) in relationship to the bound Nterminal peptide of RbcL-A. Peptide and side chains shown in stick representation. (E) Hydrogen bonds formed by the staggered pore-loops PL1 of Rca3 to Rca6 (Pro69) with the backbone of the RbcL N-terminal peptide at residues Lys11, Gln9, Lys7 and Gln5, respectively. (F) Cryo-EM density of the aromatic residues Tyr116 and Phe110 in pore-loops PL2 of Rca1 to Rca6. 

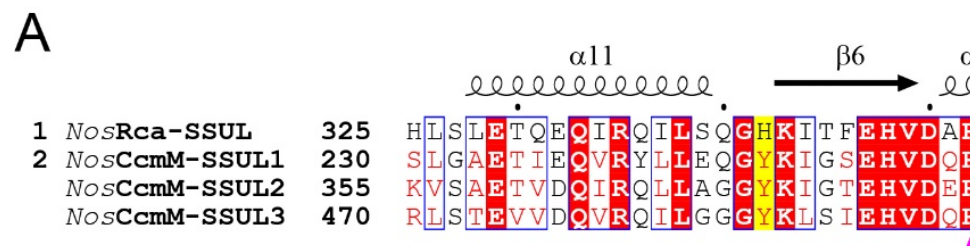

\section{2}

ele
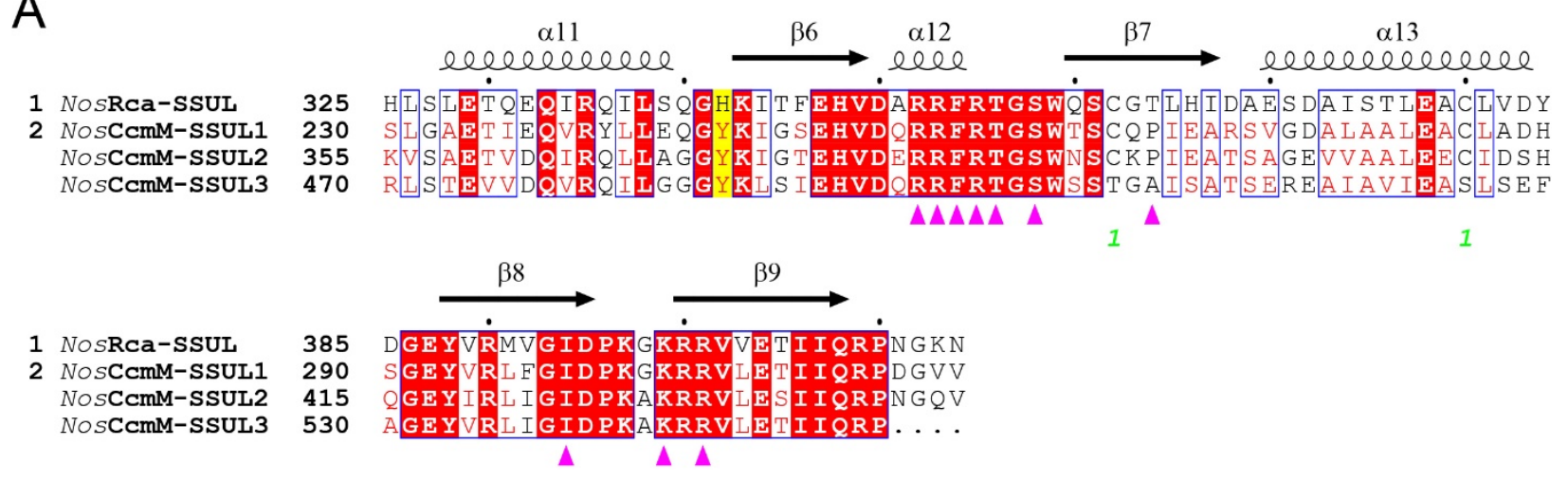

B
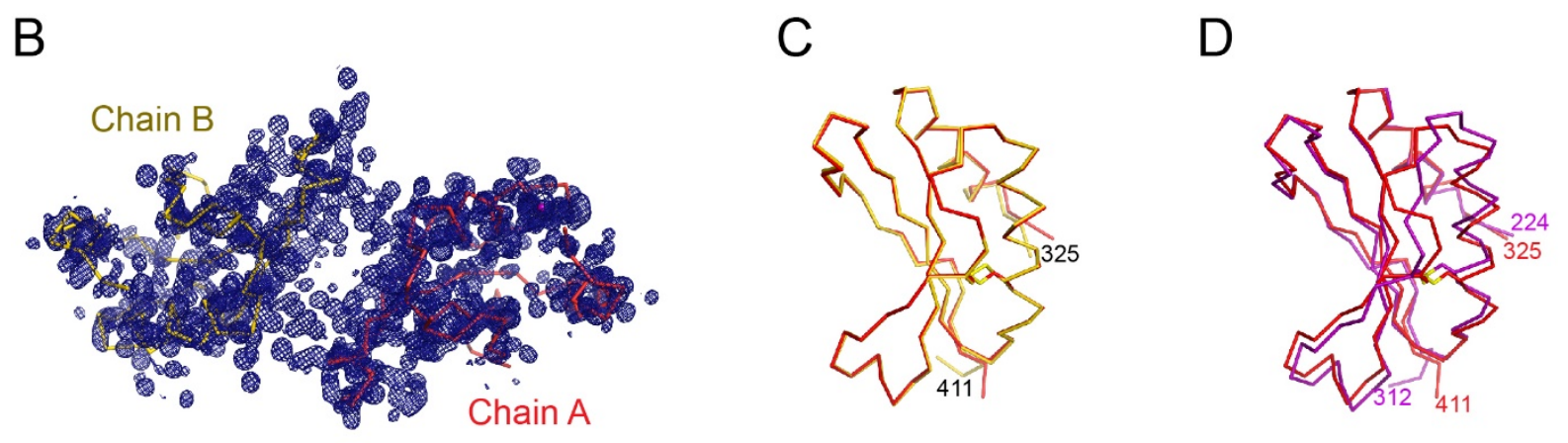

E

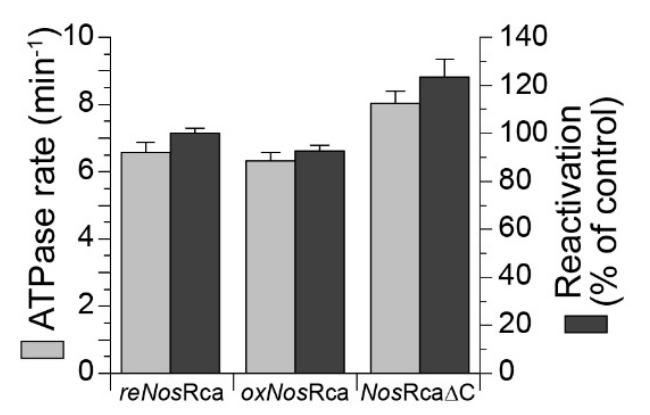

Figure S6. Role of the SSUL Domains of NosRca in Rubisco Binding.

(A) Sequence alignment of the SSUL domain of NosRca (NosSSUL) and the three SSUL modules of the scaffolding protein CcmM from Nostoc sp. PCC 7120. Secondary structure elements for NosSSUL are indicated above the sequence. Similar residues are shown in red and identical residues in white on a red background. Blue frames indicate homologous regions. Yellow background indicates systematic differences between Nos SSUL and the three SSUL modules of NosCcmM. The Uniprot accession codes for the sequences are: P58555, NosSSUL domain from Nostoc sp. PCC 7120; Q8YYI3, CcmM from Nostoc sp. PCC 7120. (B) Unbiased experimental electron density for the asymmetric unit in crystals of the NosSSUL domain at 1.4 $\AA$ resolution. The density after MAD phasing and density modification contoured at $1.5 \sigma$ is shown as a blue meshwork. The asymmetric unit contained two nearly identical copies (r.m.s.d. $0.43 \AA$ for all C $\alpha$ positions) (for data collection and refinement statistics, see Table S2). Chains A and $\mathrm{B}$ of the NosSSUL domain are shown as $\mathrm{C} \alpha$ traces in red and yellow, respectively. (C) Superposition of chain A with chain B in the asymmetric unit of the Nos SSUL crystal lattice. 
The r.m.s.d. for matching C $\alpha$ positions is $0.27 \AA$. Chain termini are indicated. (D) Superposition of the NosSSUL domain with the SSUL1 domain of CcmM from Synechococcus elongatus PCC 7942 (SeSSUL; PDB: 6HBA). The SeSSUL is shown in purple. The r.m.s.d. for matching C $\alpha$ positions is $1.0 \AA$ ( 82 matching $\mathrm{C} \alpha$ positions). (E) ATPase and reactivation rates of reduced (reNosRca), oxidized (oxNosRca) and C-terminally truncated (NosRca $\Delta \mathrm{C})$ NosRca. ATPase rates were measured in the absence of Rubisco at $20 \mathrm{mM} \mathrm{KCl}$ (see STAR Methods). $\mathrm{CO}_{2}$ fixation was measured for $8 \mathrm{~min}$ and set to $100 \%$ for reNosRca. Error bars represent SD of at least three independent experiments. 
bioRxiv preprint doi: https://doi.org/10.1101/2020.05.16.099382; this version posted May 16, 2020. The copyright holder for this preprint (which was not certified by peer review) is the author/funder, who has granted bioRxiv a license to display the preprint in perpetuity. It is made available under aCC-BY-NC-ND 4.0 International license.

A

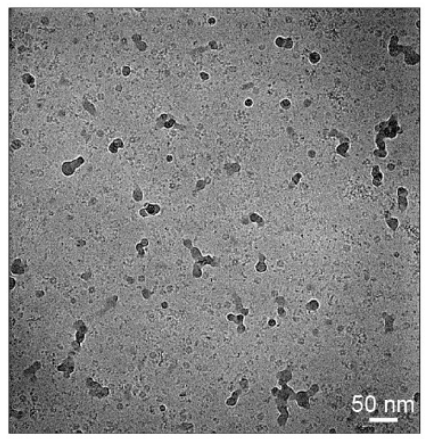

C

C 1,570 micrographs - Motioncorr2, CTFFIND4, Gautomatch - 2D classification — Initial model building $(45,859)$
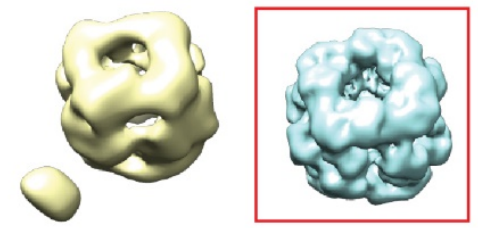

$(27,527)$

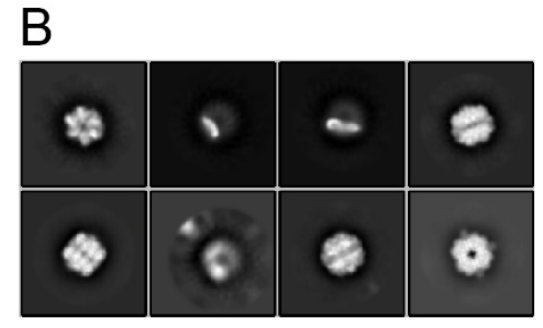

3D classification

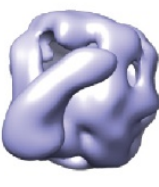

|

Focused classification on SSUL - Subtraction - Symmetry expanding - Refinement with D4
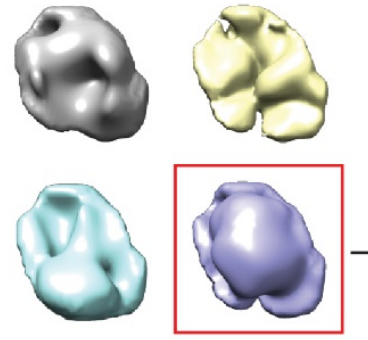

Local refinement with $\mathrm{C} 1$

$(32,128)$

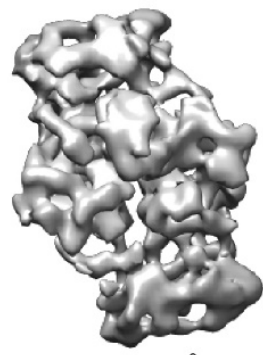

$8.2 \AA$

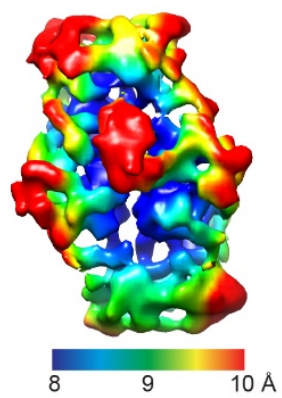

D

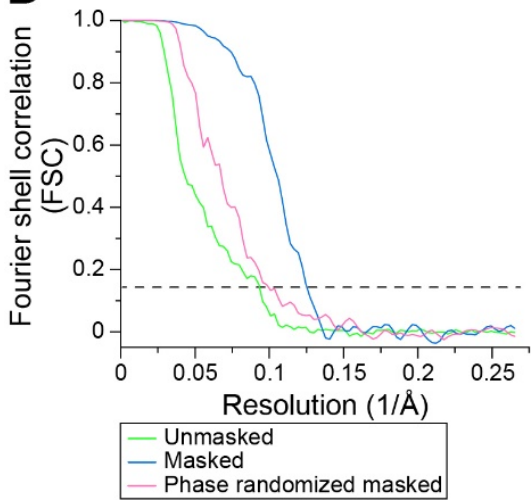

E

$\mathrm{RbcS}$

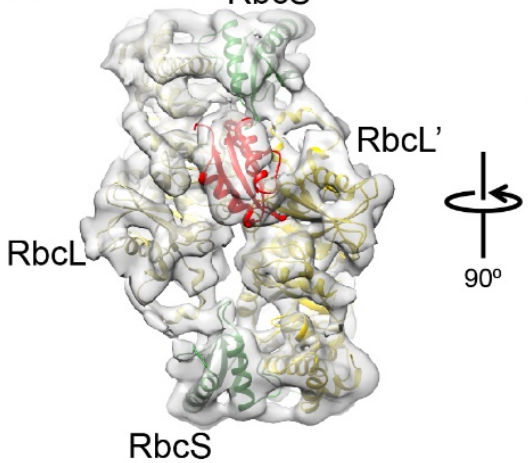

SSUL

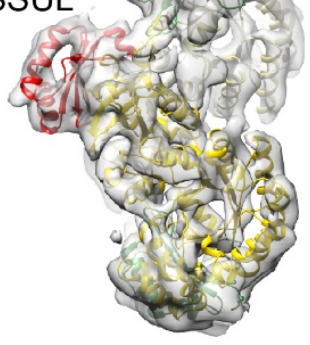


Figure S7. Cryo-EM Single-particle Reconstruction of NosRca:Rubisco Complex. (A) A representative micrograph of NosRca:Rubisco complexes. (B) 2D class averages of complexes in (A). (C) The single-particle data processing workflow for NosRca:Rubisco complex and local resolution map. The color gradient from blue to red indicates local resolution from 8.0 to $10 \AA$. (D) Gold-standard FSC curves of the NosRca:Rubisco reconstruction. The resolution is $\sim 8.2 \AA$ at the FSC cutoff of 0.143 for the masked and B-factor sharpened curve. (E) Density map of 3D reconstruction from cryo-EM and single-particle analysis of $2 \mathrm{RbcL}-2 \mathrm{RbcS}-$ SSUL units at $\sim 8.2 \AA$ resolution. The structural model in ribbon representation is docked into the cryo-EM density. The RbcL subunits in gold, RbcS in green and the bound SSUL domain in red. 
SUPPLEMENTAL TABLES

Table S1. Molar Mass and Hydrodynamic Radius of Proteins Determined by SEC-MALS.

\begin{tabular}{|c|c|c|c|c|}
\hline \multirow{2}{*}{ Protein } & \multirow{2}{*}{$\begin{array}{l}\text { Measured } \\
\text { Molar mass } \\
(\mathrm{Da}) \\
\end{array}$} & \multicolumn{2}{|c|}{ Theoretical MW (Da) } & \multirow{2}{*}{$\begin{array}{l}\text { Hydrodynamic } \\
\text { radius }(\mathrm{nm})\end{array}$} \\
\hline & & monomer & hexamer & \\
\hline NosRcaWT & $\sim 274000$ & 46668.03 & 280008.18 & $\sim 6.2$ \\
\hline NosRcaWT/ATP & $\sim 255200$ & & & $\sim 5.7$ \\
\hline NosRcaWT/ATP $\gamma \mathrm{S}$ & $\sim 255800$ & & & $\sim 6.0$ \\
\hline $\begin{array}{l}\text { NosRcaWT } \\
\text { (L244D/N248A/L250A) }\end{array}$ & $\sim 279300$ & 46453.66 & 278721.96 & $\sim 6.0$ \\
\hline $\begin{array}{l}\text { NosRcaWT } \\
\text { (V91E/R92G/G93N/ } \\
\text { L244D/N248A/L250A) }\end{array}$ & $\sim 267400$ & 46441.56 & 278649.36 & $\sim 5.9$ \\
\hline NosRca $\Delta C$ & 〜199700 & 32865.57 & 197193.42 & $\sim 5.4$ \\
\hline NosRca $\Delta$ C/ATP & $\sim 212600$ & & & $\sim 5.5$ \\
\hline NosRca $\Delta$ C/ATP $\gamma \mathrm{S}$ & $\sim 196200$ & & & $\sim 5.0$ \\
\hline $\begin{array}{l}\text { NosRca } \Delta C \\
(\text { V91E/R92G/G93N) }\end{array}$ & $\sim 195000$ & 32722.28 & 196333.68 & $\sim 5.3$ \\
\hline $\begin{array}{l}\text { NosRca } \Delta C \\
(\text { P140G/Y143A/D144A) }\end{array}$ & $\sim 197000$ & 32558.21 & 195349.26 & $\sim 5.0$ \\
\hline $\begin{array}{l}\text { NosRca } \Delta C \\
\text { (L244D/N248A/L250A) }\end{array}$ & $\sim 197400$ & 32651.2 & 195907.2 & $\sim 5.1$ \\
\hline NosRca-SSUL & $\sim 10430$ & 10938 & & $\sim 1.7$ \\
\hline
\end{tabular}


Table S2. Data Collection, Phasing and Refinement statistics.

\begin{tabular}{|c|c|c|c|c|c|c|c|c|}
\hline \multirow[b]{2}{*}{ PDB: } & \multicolumn{3}{|c|}{ NosRca $\Delta \mathrm{C}$} & \multicolumn{5}{|c|}{ NosSSUL } \\
\hline & $6 \mathrm{Z1E}$ & & 6Z1D & & $6 \mathrm{HAS}$ & & & \\
\hline & Native & & $\begin{array}{c}\mathrm{Gd}^{3+} \\
\text { complex }\end{array}$ & & $\begin{array}{c}\mathrm{Ni}^{2+} \\
\text { complex }\end{array}$ & & $\begin{array}{c}\mathrm{Ni}^{2+} \\
\text { complex }\end{array}$ & \\
\hline Data collection & & & & & & & & \\
\hline $\begin{array}{l}\text { Space group } \\
\text { Cell } \\
\text { dimensions }\end{array}$ & $H 32$ & & $H 32$ & & $P 6_{1}$ & & $P 6_{1}$ & \\
\hline$a, b, c(\AA)$ & $\begin{array}{l}112.9 \\
112.9 \\
284.3\end{array}$ & & $\begin{array}{l}111.9 \\
111.9 \\
283.6\end{array}$ & & $\begin{array}{l}59.7 \\
59.7 \\
83.3\end{array}$ & & $\begin{array}{l}\text { 60.1, } \\
60.1 \\
83.7\end{array}$ & \\
\hline$\alpha, \beta, \gamma\left({ }^{\circ}\right)$ & $\begin{array}{c}90,90 \\
120\end{array}$ & & $\begin{array}{c}90,90 \\
120\end{array}$ & & $\begin{array}{c}90,90 \\
120\end{array}$ & & $\begin{array}{c}90,90 \\
120\end{array}$ & \\
\hline & & Peak & Inflection & Remote & & Remote1 & Remote2 & Remote3 \\
\hline Wavelength $(\AA)$ & 1.1396 & 1.7108 & 1.7114 & 1.6875 & 0.9650 & 1.0723 & 1.0727 & 1.0707 \\
\hline Resolution $(\AA)$ & $\begin{array}{c}48.51 \\
-2.45 \\
(2.59 \\
- \\
2.45)^{*}\end{array}$ & $\begin{array}{c}48.99- \\
2.85 \\
(3.01- \\
2.85)\end{array}$ & $\begin{array}{c}48.98- \\
2.85 \\
(3.01- \\
2.85)\end{array}$ & $\begin{array}{c}48.99- \\
2.70 \\
(2.85- \\
2.70)\end{array}$ & $\begin{array}{c}43.95- \\
1.38 \\
(1.40- \\
1.38)\end{array}$ & $\begin{array}{c}44.19- \\
1.40 \\
(1.43- \\
1.40)\end{array}$ & $\begin{array}{c}44.20- \\
1.40 \\
(1.43- \\
1.40)\end{array}$ & $\begin{array}{c}44.21- \\
1.40 \\
(1.43- \\
1.40)\end{array}$ \\
\hline$R_{\text {sym }}$ or $R_{\text {merge }}$ & $\begin{array}{c}0.038 \\
(1.085 \\
\quad)\end{array}$ & $\begin{array}{c}0.082 \\
(0.608)\end{array}$ & $\begin{array}{c}0.074 \\
(0.543)\end{array}$ & $\begin{array}{c}0.064 \\
(0.624)\end{array}$ & $\begin{array}{c}0.065 \\
(0.922)\end{array}$ & $\begin{array}{c}0.057 \\
(1.728)\end{array}$ & $\begin{array}{c}0.043 \\
(1.008)\end{array}$ & $\begin{array}{c}0.047 \\
(1.281)\end{array}$ \\
\hline$I / \sigma I$ & $\begin{array}{l}29.3 \\
(1.9)\end{array}$ & $\begin{array}{l}30.4 \\
(5.0)\end{array}$ & $\begin{array}{l}20.0 \\
(3.4)\end{array}$ & $\begin{array}{l}37.2 \\
(5.0)\end{array}$ & $\begin{array}{l}12.5 \\
(1.4)\end{array}$ & $\begin{array}{l}30.4 \\
(1.4)\end{array}$ & $\begin{array}{l}36.5 \\
(2.5)\end{array}$ & $34.9(2.0)$ \\
\hline $\begin{array}{l}\text { Completeness } \\
(\%)\end{array}$ & $\begin{array}{c}99.8 \\
(98.6)\end{array}$ & $\begin{array}{l}99.9 \\
(99.4)\end{array}$ & $\begin{array}{c}99.9 \\
(99.6)\end{array}$ & $\begin{array}{c}99.9 \\
(99.5)\end{array}$ & $\begin{array}{c}99.6 \\
(97.5)\end{array}$ & $\begin{array}{c}99.9 \\
(98.6)\end{array}$ & $\begin{array}{c}99.8 \\
(99.1)\end{array}$ & $\begin{array}{c}99.9 \\
(99.4)\end{array}$ \\
\hline Redundancy & $\begin{array}{c}6.6 \\
(6.4)\end{array}$ & $\begin{array}{l}19.7 \\
(19.4)\end{array}$ & $8.2(8.1)$ & $\begin{array}{c}19.7 \\
(19.3)\end{array}$ & $5.1(4.6)$ & $\begin{array}{l}11.0 \\
(10.0)\end{array}$ & $\begin{array}{l}10.9 \\
(9.9)\end{array}$ & $\begin{array}{l}11.0 \\
(10.1)\end{array}$ \\
\hline Refinement & & & & & & & & \\
\hline Resolution $(\AA)$ & $\begin{array}{l}30- \\
2.45\end{array}$ & & $\begin{array}{l}30- \\
2.71\end{array}$ & & $\begin{array}{l}30- \\
1.38\end{array}$ & & & \\
\hline No. reflections & 25834 & & 19102 & & 32721 & & & \\
\hline$R_{\text {work }} / R_{\text {free }}$ & $\begin{array}{l}0.213 / \\
0.264\end{array}$ & & $\begin{array}{l}0.218 / \\
0.259\end{array}$ & & $\begin{array}{c}0.153 / \\
0.189\end{array}$ & & & \\
\hline No. atoms & & & & & & & & \\
\hline Protein & 8281 & & 8289 & & 1490 & & & \\
\hline Ligand/ion & 42 & & 44 & & 1 & & & \\
\hline $\begin{array}{l}\text { Water } \\
B \text {-factors }\left(\AA^{2}\right)\end{array}$ & 14 & & 3 & & 203 & & & \\
\hline Protein & 83 & & 78 & & 20 & & & \\
\hline Ligand/ion & 72 & & 61 & & 16 & & & \\
\hline Water & 60 & & 41 & & 29 & & & \\
\hline R.m.s deviations & & & & & & & & \\
\hline Bond lengths $(\AA)$ & 0.005 & & 0.008 & & 0.012 & & & \\
\hline Bond angles $\left(^{\circ}\right)$ & 0.992 & & 1.098 & & 1.549 & & & \\
\hline
\end{tabular}

*One crystal per structure. *Values in parentheses are for highest-resolution shell. 
Table S3. Cryo-EM Statistics and Model Validation.

\begin{tabular}{|c|c|c|}
\hline & $\begin{array}{l}\text { NosRca } \square \text { C:Rubisco } \\
\text { (EMDB: EMD-11028; } \\
\text { PDB: 6Z1F) }\end{array}$ & $\begin{array}{l}\text { NosRca:Rubisco } \\
\text { (EMDB: EMD-11029; } \\
\text { PDB: 6Z1G) }\end{array}$ \\
\hline \multicolumn{3}{|l|}{ Data collection and processing } \\
\hline Magnification & 105000 & 22000 \\
\hline Voltage (kV) & 300 & 200 \\
\hline Electron exposure (e-/ $\left./ \AA^{2}\right)$ & 60 & 47 \\
\hline Defocus range $(\mu \mathrm{m})$ & -0.7 to -2.5 & -1 to -4.5 \\
\hline Pixel size $(\AA)$ & 0.8512 & 1.885 \\
\hline Symmetry imposed & $\mathrm{C} 1$ & $\mathrm{C} 1$ \\
\hline Initial particle images (no.) & 335,474 & 298,336 \\
\hline Final particle images (no.) & 21,149 & 32,128 \\
\hline Map resolution $(\AA)$ & 2.86 & 8.2 \\
\hline FSC threshold & 0.143 & 0.143 \\
\hline Map resolution range $(\AA)$ & $2.4-23.57$ & $8.0-50.0$ \\
\hline \multicolumn{3}{|l|}{ Refinement } \\
\hline Initial model used (PDB code) & $6 Z 1 E, 1 U Z H$ & \\
\hline Model resolution $(\AA)$ & 3.2 & 8.8 \\
\hline FSC threshold & 0.5 & 0.5 \\
\hline Model resolution range $(\AA)$ & $2.7-40$ & $6.9-130$ \\
\hline Map sharpening B factor $\left(\AA^{2}\right)$ & -7.6 & -200 \\
\hline \multicolumn{3}{|l|}{ Model composition } \\
\hline Non-hydrogen atoms & 49823 & 9262 \\
\hline Protein residues & 6255 & 1168 \\
\hline \multirow{4}{*}{ Ligands } & MG: 12 & \\
\hline & CAP: 7 & \\
\hline & AGS: 5 & \\
\hline & ADP: 1 & \\
\hline \multicolumn{3}{|l|}{ B factors $(\AA 2)$} \\
\hline Protein & 65.1 & 285.1 \\
\hline Ligand & 65.4 & \\
\hline \multicolumn{3}{|l|}{ R.m.s. deviations } \\
\hline Bond lengths ( $\AA$ ) & $0.008(57)$ & $0.013(0)$ \\
\hline Bond angles $\left({ }^{\circ}\right)$ & $1.172(56)$ & $1.668(1)$ \\
\hline \multicolumn{3}{|l|}{ Validation } \\
\hline MolProbity score & 1.33 & 2.25 \\
\hline Clashscore & 2.79 & 5.98 \\
\hline Poor rotamers (\%) & 1.00 & 5.65 \\
\hline \multicolumn{3}{|l|}{ Ramachandran plot } \\
\hline Favored $(\%)$ & 96.17 & 94.89 \\
\hline Allowed (\%) & 3.72 & 5.65 \\
\hline Disallowed (\%) & 0.11 & 0.43 \\
\hline
\end{tabular}


bioRxiv preprint doi: https://doi.org/10.1101/2020.05.16.099382; this version posted May 16, 2020. The copyright holder for this preprint (which

was not certified by peer review) is the author/funder, who has granted bioRxiv a license to display the preprint in perpetuity. It is made available under aCC-BY-NC-ND 4.0 International license.

\section{SUPPLEMENTAL DATA}


bioRxiv preprint doi: https://doi.org/10.1101/2020.05.16.099382; this version posted May 16, 2020. The copyright holder for this preprint (which was not certified by peer review) is the author/funder, who has granted bioRxiv a license to display the preprint in perpetuity. It is made available under aCC-BY-NC-ND 4.0 International license.

1 Nostoc PCC 7120 Fremyella diplosiphon Geitlerinema PCC 7407 Trichodesmium erythraeum Cyanothece PCC 7425 Synechococcus PCC 7335

2 Ostreococcus lucimarinus Chlamydomonas reinhardtii Nicotiana tabacum Arabidopsis thaliana Spinacia oleracea oryza sativa
1 Nostoc PCC 7120 Fremyella diplosiphon Geitlerinema PCC 7407 Trichodesmium erythraeum Cyanothece PCC 7425 Synechococcus PCC 7335

2 Ostreococcus lucimarinus Chlamydomonas reinhardtii Nicotiana tabacum Arabidopsis thaliana Spinacia oleracea oryza sativa
1 Nostoc PCC 7120 Fremyella diplosiphon Geitlerinema PCC 7407 Trichodesmium erythraeum Cyanothece PCC 7425 Synechococcus PCC 7335

2 Ostreococcus lucimarinus Chlamydomonas reinhardtii Nicotiana tabacum Arabidopsis thaliana Spinacia oleracea oryza sativa
1 Nostoc PCC 7120 Fremyella diplosiphon Geitlerinema PCC 7407 Trichodesmium erythraeum Cyanothece PCC 7425 Synechococcus PCC 7335

2 Ostreococcus lucimarinus Chlamydomonas reinhardtii Nicotiana tabacum Arabidopsis thaliana Spinacia oleracea oryza sativa
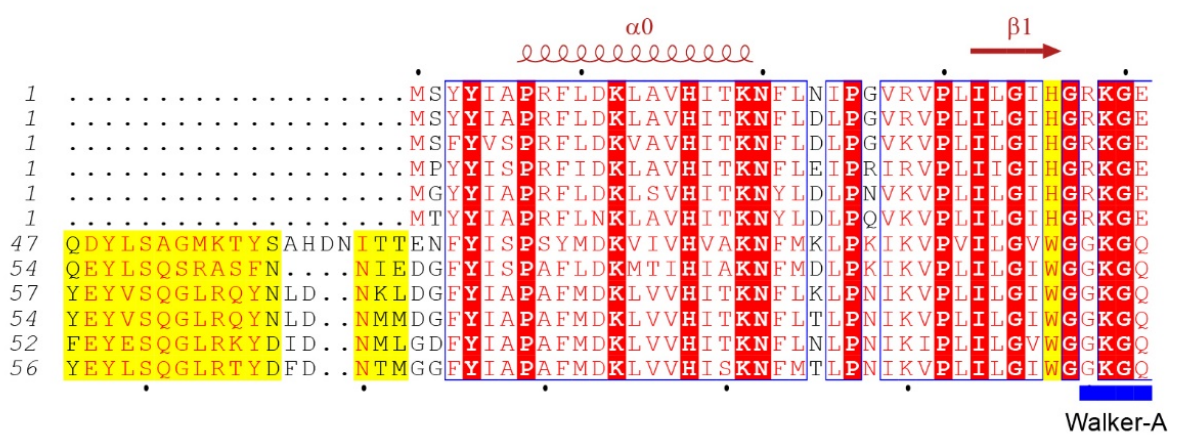$$
\text { 西 }
$$
. EQIDVDPKKQTDSDRWKGLV. . QDF DDQQDITRGKGMVDSLFQAPTGT.GTHHAVLQS
$1 . .$. $1 \ldots$ AENEEKNTDKWAHTA. KDFSDDQLDIRRGKGMVDSIFOAPADA.GTHVILS 1 . A AKLDEGKQTDQDRWKGLA. . YDISDDQQDITRGKGFVDSLFQAPTGD.GTHEAVLSS
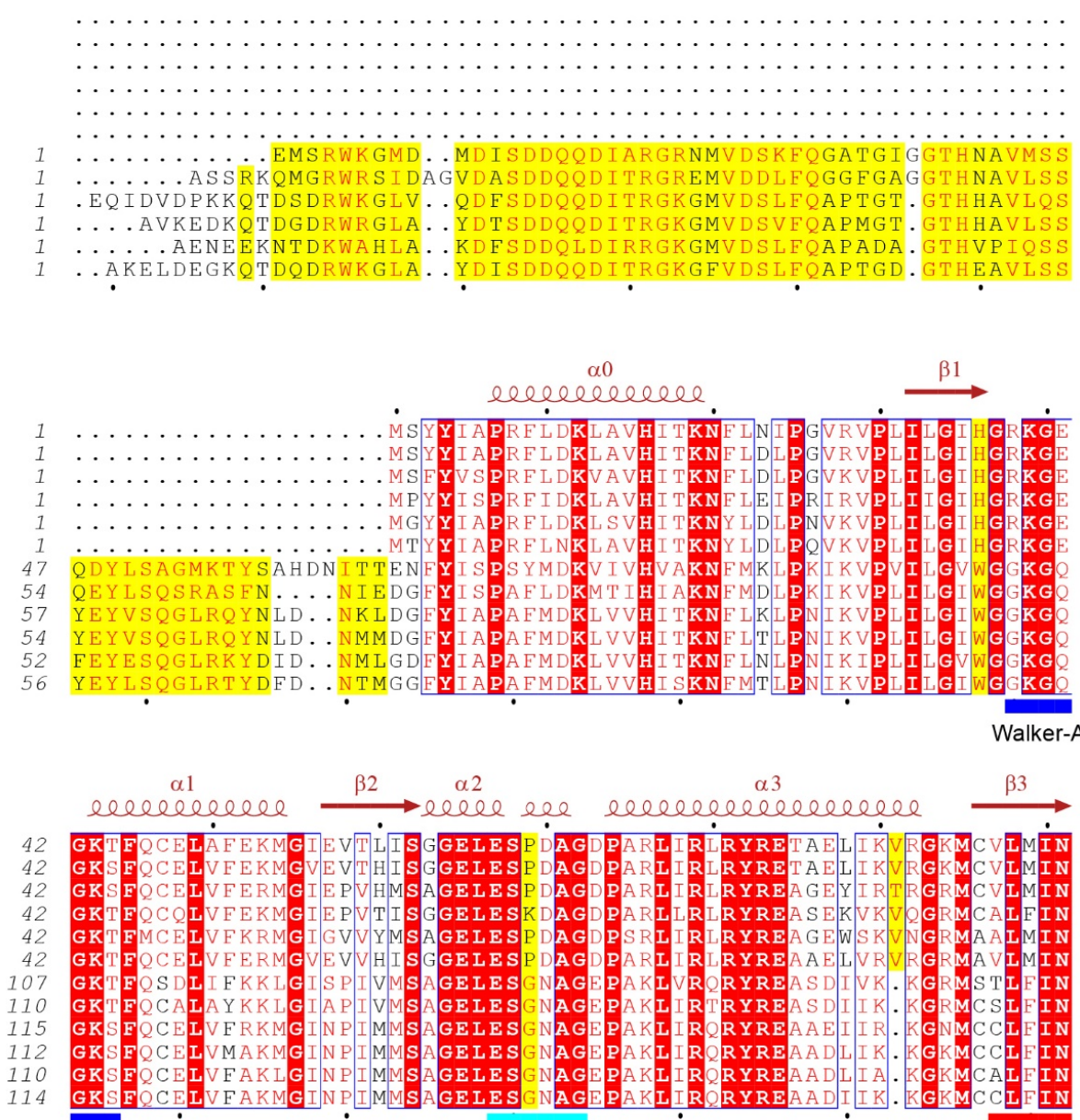

$\alpha 2$

$\alpha 3$

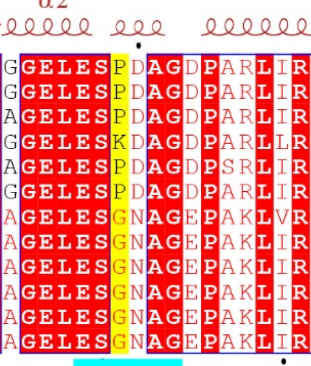

$\beta 3$
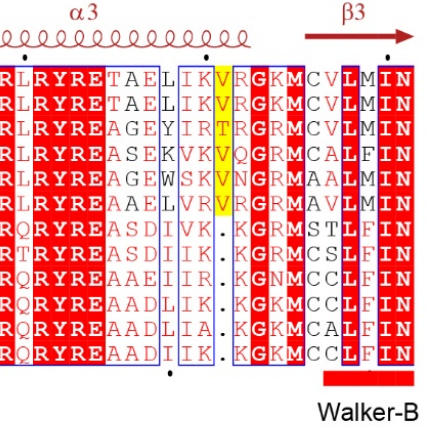

Pore loop 1

Walker-B

$\alpha 4$

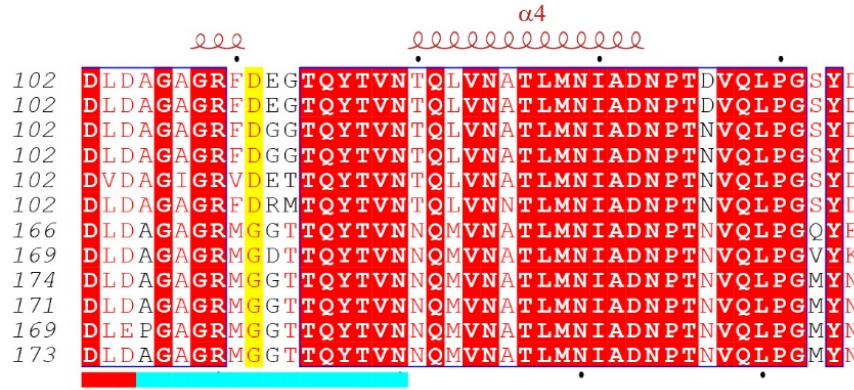

Pore loop 2

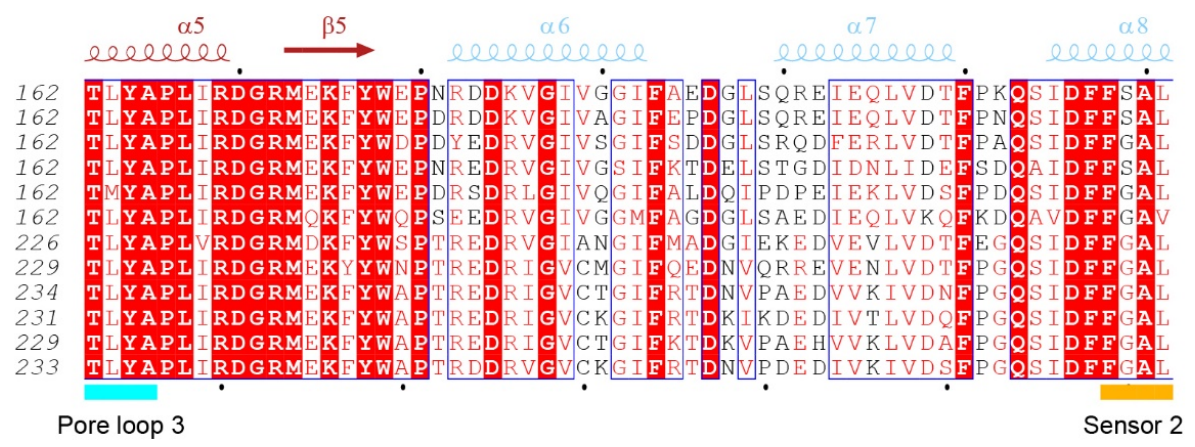

$\beta 4$

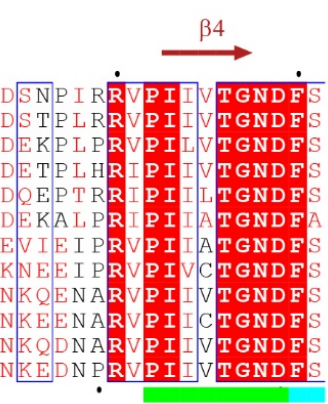

Sensor 1
1 Nostoc PCC 7120 Fremyella diplosiphon Geitlerinema PCC 7407 Trichodesmium erythraeum Cyanothece PCC 7425 Synechococcus PCC 7335

2 Ostreococcus lucimarinus Chlamydomonas reinhardti Nicotiana tabacum Arabidopsis thaliana spinacia oleracea oryza sativa 
bioRxiv preprint doi: https://doi.org/10.1101/2020.05.16.099382; this version posted May 16, 2020. The copyright holder for this preprint (which was not certified by peer review) is the author/funder, who has granted bioRxiv a license to display the preprint in perpetuity. It is made available under aCC-BY-NC-ND 4.0 International license.

1 Nostoc PCC 7120 Fremyella diplosiphon Geitlerinema PCC 7407 Trichodesmium erythraeum Cyanothece PCC 7425 Synechococcus PCC 7335

2 Ostreococcus lucimarinus Chlamydomonas reinhardtii Nicotiana tabacum Arabidopsis thaliana Spinacia olèracea oryza_sativa

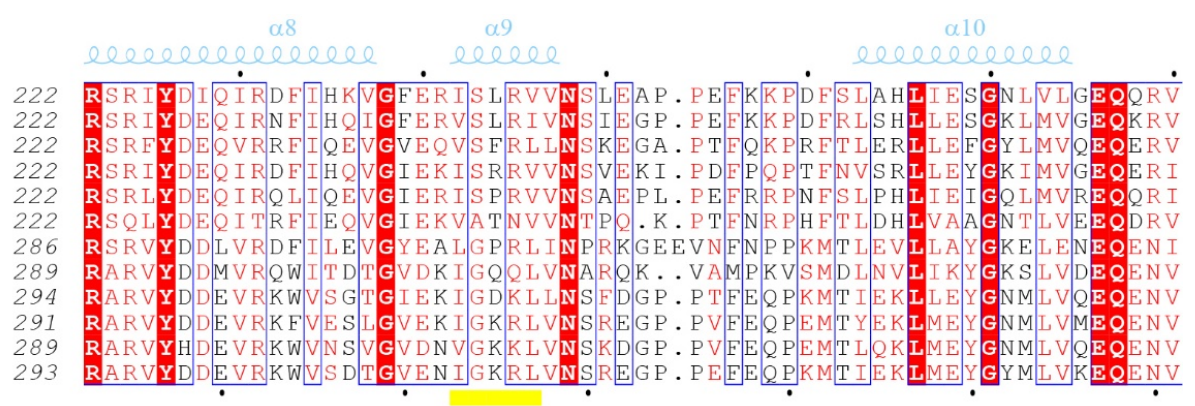

Specificity helix (H9)
1 Nostoc PCC 7120 Fremyella diplosiphon Geitlerinema PCC 7407 Trichodesmium erythraeum Cyanothece PCC 7425 Synechococcus PCC 7335

2 Ostreococcus lucimarinus Chlamydomonas reinhardtii Nicotiana tabacum Arabidopsis thaliana Spinacia oleracea oryza sativa
1 Nostoc PCC 7120 Fremyella diplosiphon Geitlerinema PCC 7407 Trichodesmium erythraeum Cyanothece PCC 7425 Synechococcus PCC 7335

2 Ostreococcus lucimarinus Chlamydomonas reinhardtit Nicotiana tabacum Arabidopsis thaliana Spinacia oleracea oryza sativa
1 Nostoc PCC 7120 Fremyella diplosiphon Geitlerinema PCC 7407 Trichodesmium erythraeum Cyanothece PCC 7425 Synechococcus PCC 7335

2 Ostreococcus lucimarinus Chlamydomonas reinhardtii Nicotiana tabacum Arabidopsis thaliana Spinacia oleracea oryza sativa

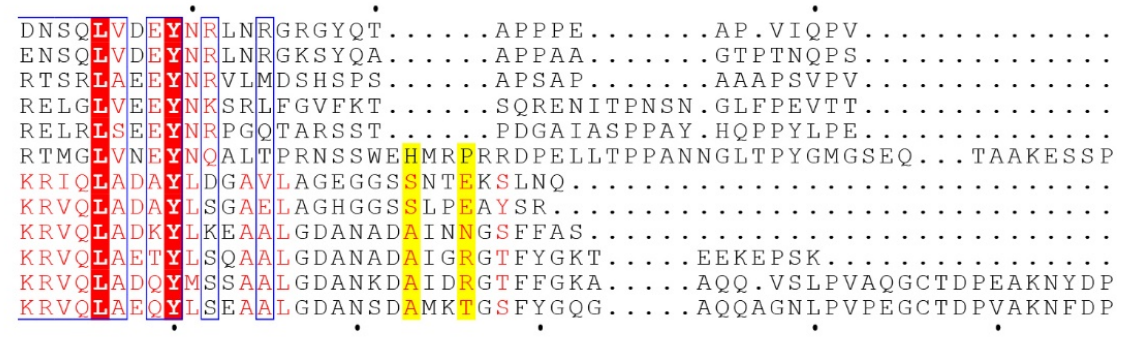

$\alpha 11$

elelelelel

$\beta 6 \quad \alpha 12$

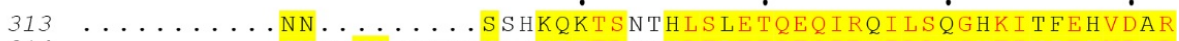
$314 \ldots \ldots . \ldots$ SNGVNKGATNHN LQKPETLSPHLTLETQEQIRQI LAQGYTIN I EHVDER

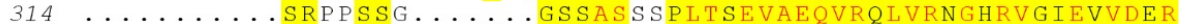

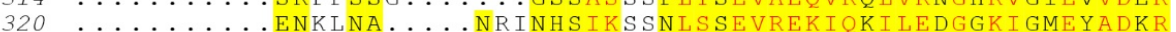
$320 \ldots \ldots \ldots$. . . . . . S S S H S S A A P SEPGS L S PEVEQQLIGLVRQGQRIGLEYADPR 337 WQRLREARIARS IQP S SNANGSEQPSRSS L S L L S TVQQQLQSVLSSGDR I A IEHSDQR

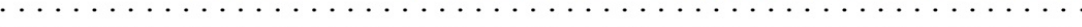

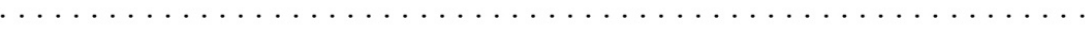

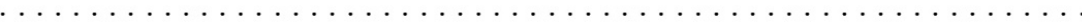

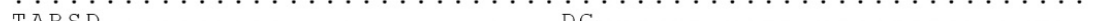

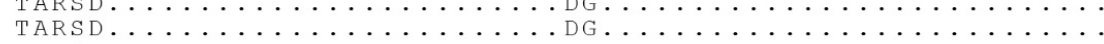

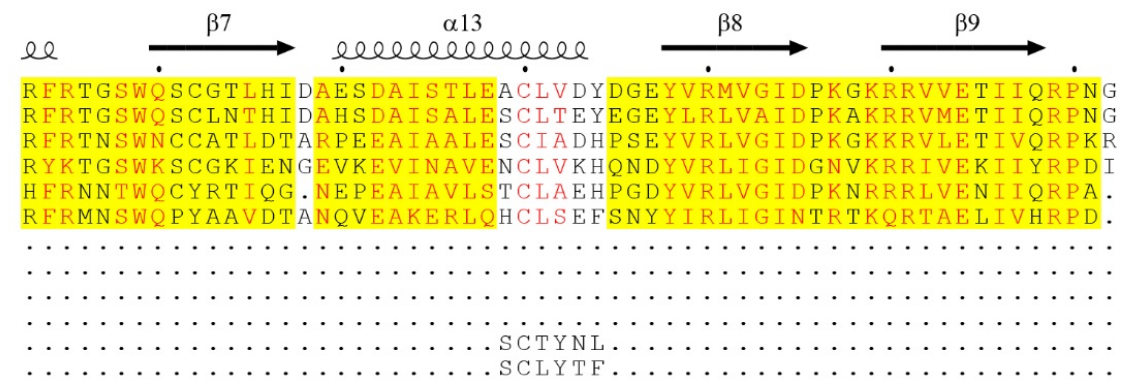

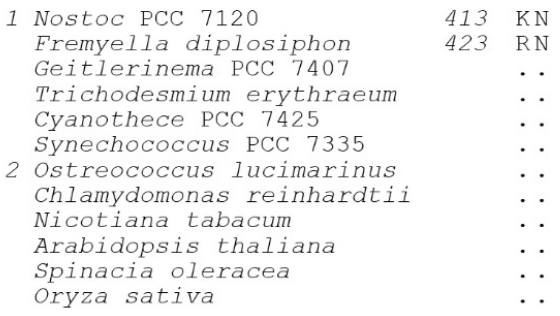


Data S1. Alignment of Cyanobacterial 'Rca-like' and Plant Rca Sequences. Amino acid sequences of representative sets of cyanobacterial 'Rca-like' (group 1) and eukaryotic green lineage Rca proteins (group 2) were aligned using the EBI Clustal-O server (https://www.ebi.ac.uk/Tools/msa/clustalo/). Secondary structure elements for Rca from Nostoc sp. PCC 7120 are indicated above the sequences. Similar residues are shown in red and identical residues in white on a red background. Blue frames indicate homologous regions. Yellow background indicates systematic differences between cyanobacterial and eukaryotic sequences. Canonical elements of AAA + domains are indicated by colored bars: Dark blue, Walker-A motif; red, Walker-B motif; green, sensor 1; orange, sensor 2. Teal bars indicate pore-loops PL1, PL2 and PL3. The yellow bar denotes the specificity helix H9. The Uniprot and Genebank accession codes for the sequences are: P58555, Nostoc sp. PCC7120; NZ JH930359.1, Fremyella diplosiphon; K9SEV2, Geitlerinema PCC7407; Q115H0, Trichodesmium erythraeum; B8HYF8, Cyanothece PCC7425; B4WNZ5, Synechococcus PCC7335; D8TZU3, Volvox carteri; A4RW20, Ostreococcus lucimarinus; Q6SA05, Chlamydomonas reinhardtii; Q40460, Nicotiana tabacum; P10896, Arabidopsis thaliana; P10871, Spinacia oleracea; P93431, Oryza sativa. The Figure was generated with ESPript (Gouet et al., 1999). 
bioRxiv preprint doi: https://doi.org/10.1101/2020.05.16.099382; this version posted May 16, 2020. The copyright holder for this preprint (which was not certified by peer review) is the author/funder, who has granted bioRxiv a license to display the preprint in perpetuity. It is made available under aCC-BY-NC-ND 4.0 International license.

NosRca NtRca

AtRca
1

... AVKEDKQTDGDRWRGLAYDTSDDQQDITRGKGMVDSVFQAPMGTGTHHAVLSSYEYV
NosRca 48

NtRca 121

AtRca 118 $\alpha 0$

elelelelelel.

NtRca

AtRca

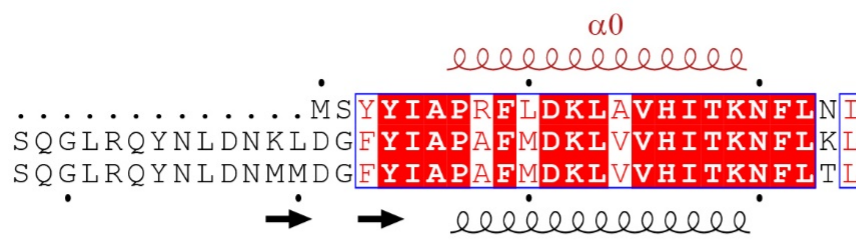

$\rightarrow \rightarrow$ eleibelebel $^{\circ}$

$\alpha 3$

elelelelelelelel

$\stackrel{\beta 1}{\longrightarrow}$

elele

P LILGIHGRK $\dot{E} E G K T F Q C$ V RV P L I LG I H R R GE GK FQC
I KVP L I L I IWGGK GQGKS F Q C KV P L I L G I W G GK GQGKS F Q C

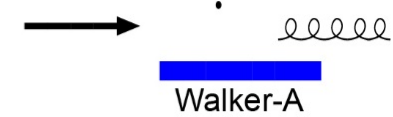

$\alpha 1$

$\beta 2 \quad \alpha 2$

epe

$\beta 3$

belele

IE

VIISGGLESP

KLIR RYR A I LAGA

ELVF R KMG IN P IMMSAGELESGNAGEPA ELVMAKMG IN P IMM SAG LESGNAGEPAKLIRQRYREAADLIK. KGKMCCLFINDLDAGA elelel

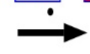

lelebelelele

Pore loop 1

Walker-B

$\alpha 4$

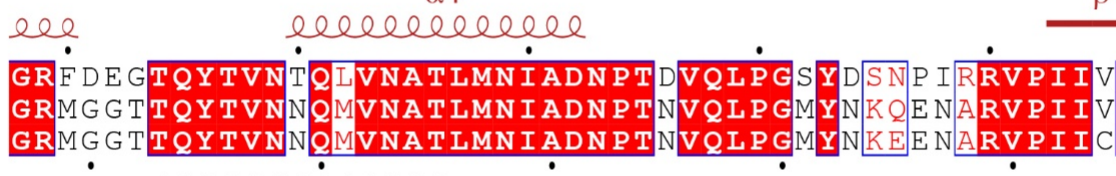

$\beta 4$

H5

beblebelel

$\longrightarrow$

NosRca 108

AtRca 177

\section{Pore loop 2}

Sensor 1 Pore loop 3

NosRca 168

NtRca 240

AtRca 237

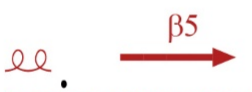

$\stackrel{\beta 5}{\longrightarrow}$

$\alpha 6$

IRD GRMEKFYW

IRDGRMEKFYWAPTREDR I GVVTGIFRTDNVIPA E DVVVIVDNFP

I RD GRMEKF YWAPTREDR I GVCKG I FRTDKIK DEDIVTLVDQF P GQS I D F F GA L RARVYD

$\mathrm{el} \longrightarrow$ elebeleie ilebelee

elebelebele

Sensor 2

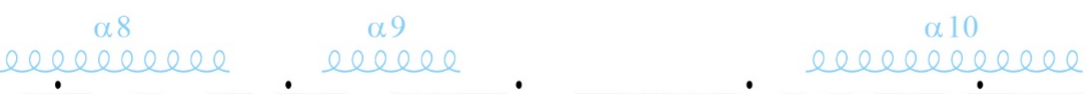

NosRca 228

NtRca 300

AtRca 297

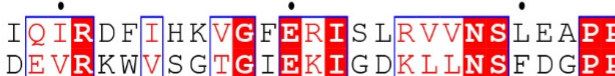

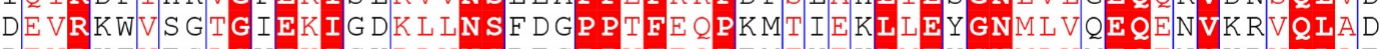

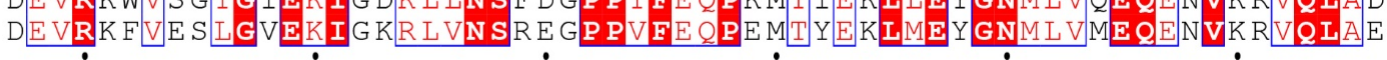

elebelel elebel

beblebelebelebelele

Specificity helix (H9)

NosRca 288

NtRca 360

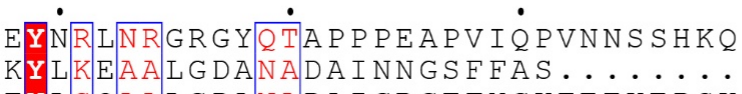

TYLSQAALGDANADA I GRGTFYGKTEEKEP S K 
bioRxiv preprint doi: https://doi.org/10.1101/2020.05.16.099382; this version posted May 16, 2020. The copyright holder for this preprint (which

was not certified by peer review) is the author/funder, who has granted bioRxiv a license to display the preprint in perpetuity. It is made available under aCC-BY-NC-ND 4.0 International license.

Data S2. Sequence Alignment of NosRca, NtRca and AtRca.

Amino acid sequences of NosRca, NtRca and AtRca were aligned using the EBI Clustal-O server (https://www.ebi.ac.uk/Tools/msa/clustalo/). Secondary structure elements for NosRca and plant Rca proteins are indicated above and below the sequences, respectively. Similar residues are shown in red and identical residues in white on a red background. Blue frames indicate homologous regions. Canonical elements of AAA+ domains are indicated by colored bars: Dark blue, Walker-A motif; red, Walker-B motif; green, sensor 1; orange, sensor 2. Teal bars indicate pore-loops PL1, PL2 and PL3. The yellow bar denotes the specificity helix H9. The Uniprot accession codes for the sequences are: P58555, Nostoc sp. PCC 7120; Q40460, Nicotiana tabacum and P10896, Arabidopsis thaliana. 